

\section{DISCLAIMER}

This report was prepared as an account of work sponsored by an agency of the United States Government. Neither the United States Government nor any agency Thereof, nor any of their employees, makes any warranty, express or implied, or assumes any legal liability or responsibility for the accuracy, completeness, or usefulness of any information, apparatus, product, or process disclosed, or represents that its use would not infringe privately owned rights. Reference herein to any specific commercial product, process, or service by trade name, trademark, manufacturer, or otherwise does not necessarily constitute or imply its endorsement, recommendation, or favoring by the United States Government or any agency thereof. The views and opinions of authors expressed herein do not necessarily state or reflect those of the United States Government or any agency thereof. 


\section{DISCLAIMER}

Portions of this document may be illegible in electronic image products. Images are produced from the best available original document. 


\section{DISCLAIMER}

"This book was prepared as an account of work sponsored by an agency of the United States Government. Neither the United States Government nor any agency thereof, nor any of their employees, makes any warranty, express or implied, or assumes any legal liability or responsibility for the accuracy, completeness, or usefulness of any information, apparatus, product, or process disclosed, or represents that its use would not infringe privately owned rights. Reference herein to any specific commercial product, process, or service by trade name, trademark, manufacturer, or otherwise, does not necessarily constitute or imply its endorsement, recommendation, or favoring by the United States Government or any agency thereof. The views and opinions of authors expressed herein do not necessarily state or reflect those of the United States Government or any agency thereof."

This report has been reproduced directly from the best available copy.

Available from the National Technical Information Service, U. S. Department of Commerce, Springfield, Virginia 22161.

Price: Paper Copy $\$ 10.00$

Microfiche $\$ 3.50$ 


\title{
ENERGY USE IN OFFICE BUILDINGS
}

\author{
Department of Energy Contract \\ DOE DE-AC01-79CS 20189 \\ Volume 1
}

\begin{abstract}
Analysis of 1977 Office Building
Energy Use as Reported in the

Building Owners and Managers Association Data Base
\end{abstract}

August 29, 1980

\section{General Electric Company \\ Energy Analysis \\ 777 14th Street, N.W. \\ Washington, DC. 20005}




\section{PREFACE}

This is the report on Task IA; Analysis of the 1977 BOMA Data Base; of the General Electric Company's Energy Use in Office Buildings project; work conducted under a cost sharing contract with the Department of Energy. The analysis and calculations in this volume were performed by G.E.'s Energy Analysis Section of the Power Systems Strategic Planning and Development Operation.: The report was prepared for the Department of Energy's Division of Buildings' and Community "Systems, Office of Conservation and Solar.

The project was undertaken in the hope of filling' a major gap in the available data on energy consumption. Survey and census data that can be used for estimating energy consumption are published regularly for the residential and industrial sectors of the economy. Similar data do not yet exist for the commercial sector, and it will probably be several years before all of the data from a major DOE/EIA commercial sector energy survey which is currently in process will have been published and analyzed.

General Electric's earlier efforts in this area, the Commercial Sector Energy Consumption Data Base Development Project, developed estimates on the square feet of commercial space and assembled existing data on the energy consumption characteristics of the space. Also in that earlier project, an initial effort was made to provide greater detail on energy consumption in office buildings. Three reports were produced:

- Commercial Buildings Inventory, General Electric, Center for Energy Systems, Volume I, DOE Contract EC-77-C-01-2161, June 1978.

- Survey of Available Energy Use Data, General Electric, Center for Energy Systems, Volume II, DOE Contract EC-77-C-01-2161, April 1978.

- Analysis of Energy Consumption in Office Buildings, General Electric, Center for Energy Systems, Volume III, DOE Contract EC-77-C-012161, April 1978. 
The current project, Energy Use in Office Buildings, builds on the information developed in the initial project. The work is focused on office buildings, which are a major and complex energy consuming subsector of the Commercial Sector. As currently defined the contract has three major outputs.

- The verification and tabular presentation of data from the 1977 commercial office building data base of the Building Owners and Managers Ássociation (BOMA).

- A tabular and regression analysis of a new and significantly expanded 20 city of fice building data base recently developed by BOMA.

- A review and analysis of a 400 building supplementary survey conducted by BOMA.

This volume presents the results of the analysis of the 1977 BOMA data base. When the project has been completed it is expected that the information on office building energy use and the factors which drive energy use will have been substantially improved. 


\section{TABLE OF CONTENTS}

Section

$\underline{\text { Page }}$

PREFACE

iii

SUMMARY

ix

INTRODUCTION

1

I THE BOMA ORGANIZATION AND DATA BASE

The 1977 Data Base: Conclusions

II ANALYSIS OF THE 1977 DATA BASE: COMMERCIAL

OFFICE BUILDINGS

II-1 Characteristics of the Data Base $\quad 8$

II-2. Summary of Energy Consumption 14

II-3 Energy Consumption Comparison: Downtown/Suburban Use 27

II-4 Energy Consumption by Height 41

II-5 Energy Consumption by Building 44

II-6 Electric Billing Policy of Management 47

II-7 Energy Consumption and Computer Space . 50

II-8 Energy Consumption and Building Air Conditioning . 55

II-9 Conclusions on Commercial Office Building Energy Consumption 60

III ANALYSIS OF THE 1977 DATA BASE: GOVERNMENT OFFICE BUILDINGS

III-1 Characteristics of the Data Base 62

III-2 Summary of Energy Consumption 66

III-3 Energy Use Comparison: Downtown/Suburban 79

III-4 Energy Consumption by Building Height $\quad 86$

III-5 Energy Consumption by Building Age 88

III-6 Energy Consumption and Computer Space 91

III-7 Energy Consumption and Building Air Conditioning Equipment 95

III-8 Conclusions on Energy Use in Government Office Buildings , 100

IV CONCLUDING COMMENTS 101

APPENDIX $1 \quad 103$

APPENDIX 2 


\section{TABLES BY SECTION OF REPORT}

II. Analysis of the 1977 Data Base: Commercial Office Buildings

Table Number

Title

Page

II-1 Percentage Distribution of Square Feet of Commercial Office Building Space by Census Division

II-2 Percentage Distribution of Square Feet of Commercial Office Building Space by Age

II-3 The 1977 BOMA Office Building Sample by Type of Heating Fuel and Region, Commercial Office Buildings

II-4 Heating Fuel by Age of Building in the 1977 BOMA Office Building Sample, Commercial Office Buildings

II-5 Energy Consumption in the 1975 and 1977 Commercial Office Building Samples by Type of Heating Fuel

II-6 Confidence Intervals for the 1977 Mean for Commercial Office Buildings

II-7

Summary of 1977 Commercial Office Building Energy Consumption

II-8 Energy Consumption in the 1977 BOMA Office Building Sample, Commercial Office Buildings

II-9 Energy Consumption in the 1977 BOMA Office Building Sample; Electrically Heated Buildings, Commercial Office Buildings

II-10 Energy Consumption in the 1977 BOMA Office Building Sample, Gas Heated Buildings, Commercial Office Buildings

II-11 Energy Consumption in the 1977 BOMA Office Building Sample, Oil Heated Buildings, Commercial Office Buildings

II-12

Energy Consumption in the 1977 BOMA Office Building Sample, Steam Heated Buildings, Commercial Office Buildings

II-13 Comparison of Energy Consumption: Downtown and Suburban Commercial Office Buildings

II-14 Downtown and Suburban Buildings in the 1977 BOMA Office Building Sample, Commercial Office Buildings

II-15 Energy Consumption on a Weighted Basis by Building Height, 1975 and 1977, Commercial Office Buildings 
II. Analysis of the 1977 Data Base: Commercial Office Buildings

Table Number

Title

Page.

II-16 Energy Consumption in the 1977 BOMA Office Building Sample by Building Height, Commercial Office Buildings

II-17 Energy Consumption on a Weighted Basis by Building Age, Commercial Office Buildings

II-18 Energy Consumption in the 1977 BOMA Office Building Sample by Building Age, Commercial Office Buildings

II-19 Electric Bill Policy of Building Management In 1977 BOMA Office Building Sample, Commercial Office Buildings

II-20 Energy Consumption in the 1977 BOMA Office Building Sample, Buildings with Computer Space, Commercial Office Buildings

II-21 Energy Consumption in the 1977 BOMA Office Building Sample, Buildings without Computer Space, Commercial Office Buildings

II-22 Energy Consumption in the 1977 BOMA Office Building Sample, Buildings with Electric Air Conditioning, Commercial Office Buildings

II-23 Energy Consumption in the 1977 BOMA Office Building Sample, Buildings with Gas Air Conditioning, Commercial Office Buildings

II-24 Energy Consumption in the 1977 BOMA Office Building Sample, Buildings with Steam Absorption Air Conditioning, Commercial Office Buildings

II-25 Energy Consumption in the 1977 BOMA Office Building Sample, Buildings with Steam Turbine Air Conditioning, Commercial Office Buildings

III. Analysis of the 1977 Data Base: Government Office Buildings

III-1 Percentage Distribution of Square Feet of Government Office Building Space by Census Division

III-2 Percentage Distribution of Square Feet of Government Office Building Space by Age

III-3 The 1977 BOMA Office Building Sample by Type of Heating Fuel and Region, Government Office Buildings

III-4 Heating Fuel by Age of Building in the 1977 BOMA Office Building Sample, Government Office Buildings 
II. Analysis of the 1977 Data Base: Commercial Office Buildings

III-5 . Comparison of Energy Consumption Between the Commercial and Government Office Buildings by Type of Heating Fuel

III-6

Summary of 1977 Government Office Building Energy Use

III-7 Energy Consumption in the BOMA Office Building Sample, Government Office Buildings

III-8 Energy Consumption in the 1977 BOMA Office Building Sample, Electrically Heated Buildings, Government Office Buildings

III-9 Energy Consumption in the 1977 BOMA Office Building Sample, Gas Heated Buildings, Government Office Buildings

III-10 Energy Consumption in the 1977 BOMA Office Building Sample, Oil Heated Buildings, Government Office. Buildings

III-11 Energy Consumption in the 1977 BOMA Office Building Sample, Steam Heated Buildings, Government Office Buildings

III-12 Downtown and Suburban Buildings in the 1977 BOMA Office Building Sample, Government Office Buildings

III-13 Energy Consumption in the 1977 BOMA Office Building Sample by Building Height, Government Office Buildings

III-14 Comparison of Energy Use by Age in Commercial and Government Office Buildings

III-15 Energy Consumption in the 1977 BOMA Office Building Sample by Building Age, Downtown Buildings, Government Office Buildings

III-16 Energy Consumption in the 1977 BOMA Office Building Sample, Buildings with Computer Space, Government Office Buildings

III-17 $\quad \because \quad$ Energy Consumption in the 1977 BOMA Office $\because$ Building Sample, Buildingś: without Computer Space, Government Office Buildings

III-18 . Energy Consumption in the 1977 BOMA Office Building Sample, Buildings with Electric Air Conditioning Government Office Buildings 


\section{TABLES BY SECTION OF REPORT, CONTINUED}

II. Analysis of the 1977 Data Base: Commercial Office Buildings

III-19

Energy Consumption in the 1977 BOMA Office Building Sample, Buildings with Gas Air Conditioning, Government Office Buildings

III-20

Energy Consumption in the 1977 BOMA Office Building Sample, Buildings with Steam Absorption Air Conditioning, Government Office Buildings 
This report presents the results: of Task IA of the Energy Use in Office: Buildings Project: An analysis in tabular form of the 1977 office building energy use data base of the Building Owners and Managers Association (BOMA). ${ }^{1}$ ) BOMA's approximately 4000 members directly manage over 500 million square feet of commercial office space, which is approximately $16 \%$ of total commercial office building space. BOMA annually collects data on office building characteristics and operating performance for presentation in its Experience Exchange Report. Data are collected from BOMA member and non-member buildings electing to participate in the reporting process; and, in addition, a number of Federal, state, and local government buildings have been participating since 1977. Summaries of the data are published by BOMA on an aggregate basis; the summaries, which are developed on a city or regional basis, provide a benchmark for use by building managers in comparing the results of specific building operations with the industry's aggregate experience.

The BOMA Experience Exchange Report data base is believed to be the most comprehensive commercial office building data base available. It is, however, subject to some constraints. The buildings which participate in the survey are believed to be better maintained and more professionally managed than the average commercial office building; i.e., the data base is largely composed of prime office space. To the degree that non-prime space is under represented, there may be a bias in extrapolating the results to office buildings in general. There is no information available, however, about the amount of non-prime office space in the office building population; some information on this is being developed under a separate task of the Energy Use in, Office Buildings Project. Second, since the Experience Exchange Report is a voluntary report, the data are probably less accurate than those which would be obtained from a mandatory report.

1) The Energy Use in Office Buildings Project was conducted under a cost sharing contract with the Department of Energy, DOE DE-AC01-79CS 20189. 
Access to the 1977 BOMA data base was obtained under a subcontract with BOMA. Data for 1342 buildings -- 1059 commercial office buildings and 283 government office buildings in the United States and Canada -- were delivered. Of the 1059 commercial office buildings, 999 were located in the United States. A total of 233 Federal, state, and local government operated buildings located in the United States were also in the data base.

Prior to analysis the data were subjected to an intensive review. Questions were referred to the BOMA staff for explanation or correction. In some cases the BOMA staff were able to verify or correct a data item from existing records. In other cases it was necessary for the BOMA staff to contact individual building managers. In some cases it was impossible for a building manager to verify or correct a data item, and in other cases BOMA could not reach the appropriate person. A number of buildings were, therefore, eliminated from the energy analysis.

After the Canadian buildings and the buildings with questionable data had been eliminated, a sample consisting of 889 commercial and 216 government office buildings for which data had been verified was finally developed from the data furnished by BOMA. The sample of buildings will be referred to in this report as the "1977 data base", to distinguish it from the somewhat larger data base from which it was obtained, the 1977 Experience Exchange Report.

Energy use data were reported by BOMA in terms of kwh of electricity, cubic feet of gas, gallons of oil, and pounds of steam. The data were converted to British thermal units (Btu's), and all building energy measures were expressed in terms of Btu/Sq. Ft.

Section II of the report presents the analysis for commercial office buildings; and Section III presents the analysis for government of fice buildings.

On the basis of data previously developed from the F.W. Dodge "Dodge Construction Potentials" and presented in Commercial Buildings Inventory, it was found that the commercial office buildings in the data base were reasonably representative of the stock of commercial office buildings in terms of location and age. 1) Tables summarizing electric consumption and fuel consumption by fuel and census division, downtown/suburban location, height, age, electrical billing policyof management, computer space, and air conditioning were developed.

1) General Electric Company, Center for Energy Systems, Commercial Buildings Inventory, June 1978. 
As measured by square feet of space $15 \%$ of the commercial buildings were electrically heated, $35 \%$ were gas heated, $7 \%$ were oil heated, and $42 \%$ were steam heated. Electrically heated buildings used the least energy per square foot in 1977 in comparison to other types of buildings. Steam heated buildings were the second most energy efficient, followed by oil heated buildings. Gas heated buildings were the least energy efficient. (The data are summarized in Table II-5). Both electricity and fuel were found to vary by census division. Fuel consumption tended to be higher in cold regions, and electric consumption tended to be higher in warm regions (Tables II-8 through II-12).

There was found to be a difference in energy use between downtown and suburban office buildings: on an aggregate basis downtown buildings were found to use more energy per square foot than suburban buildings (Tables II-13 and II-14).

In examining energy use by height there was no clear energy use trend in the case of fuel use, but buildings in excess of 9 stories used more electricity per square foot than did lower buildings. (Tables II-15 and II-16). This may be due to relatively greater elevator and air handling needs in the taller buildings.

Energy use was found to be a function of building age (Tables II-17 and II-18). Energy use is relatively low in new buildings, probably as the result of the buildings having heating, ventilating, and air conditioning equipment which is in good operating condition. Energy use is higher for buildings in the 10-19 years old range, possibly due to older and less efficient equipment. Energy use then tapers off to a low level for buildings through the 30-39 years age range, as the quality of the space declines. Buildings in the 40+ years old range have generally been remodeled, and their energy use reflects that the space is again high quality, prime space. Relatively low energy use in the $50+$ years old buildings probably is due to the construction practices of the 1920's and to the use of steam for heating.

Charges for electricity are usually included in the building rent, and an escalator clause typically provides for the pass-through of increased energy charges for a building in proportion to the space rented by each tenant. Under these circumstances the tenant has little control over the charge to him for electricity. In some buildings, however, the tenant is metered by the building or by the electric utility for some of his electric use -- such as lighting, computer, and general office uses - and can lower his electric bill through decreased electric use. It was found that in cases in which tenants were metered for their electric use and could directly benefit financially from conservation, the buildings tended to use less electricity (Table II-19). 
The existence of computer or data processing facilitles in a bullding was found to have an effect on building energy use: buildings without computer space used more fuel and less electricity on average than did buildings with computer space (Tables II-20 and II-21). A possible explanation of thls would be that large amounts of electricity are used to power the computer equipment and that the waste heat subsequently filters through the rest of the building, reducing some of the need for heating fuel use. Given the zoning of many commercial buildings, however, it appears that general building usage patterns rather than waste heat. recovery may also be an important factor in explaining energy use in this case.

Data on energy use and air conditioning were also developed (Tables II-22 through II-25). Electric consumption for buildings with non-electric air conditioning (steam absorption, gas, etc.) was significantly less than for buildings with electric air conditioning.

Section III presents the office building energy use analysis for government office buildings, defined to be buildings owned and operated by a government agency. There were 216 government office buildings in the 1977 data base. The buildings were not, however, typical of government office buildings in general in terms of location or age. In terms of heating fuel, $5 \%$ of the space was electrically heated, $36 \%$ was gas heated, $8 \%$ was oil heated, and $51 \%$ was steam heated. In contrast to the commercial office buildings in the 1977 data base, the government buildings tended to fall in the middle age ranges: there were fewer old (50 years +) or younger ( $1-9$ years) buildings.

Government office buildings were found to use less energy than do commercial office buildings (Tables III-5 through III-11). Differences in building construction and building usage could account for the lower energy consumption. The government architectural style results in building design which is structurally. more massive than commercial space and probably has better thermal characteristics. In addition, building managers for government buildings have frequently received directions to operate the buildings at comfort levels for lighting, heating, and cooling which are below those maintained in the private sector. Commercial building managers in providing space conditioning have to maintain a level of tenant satisfaction and comfort that will enhance the marketability of the space. In contrast, government building managers in some instances operate the buildings pursuant to government conservation policies. 
An attempt was made to compare energy use between downtown and suburban government office buildings. (Table III-12). There is some evidence that suburban . government gas and oll heated bulldings use more energy than do downtown government buildings. However, since most government buildings are located downtown, the data are too limited to permit definite conclusions.

Table III- 13 presents energy use by building height; there did not appear to be any significant trend in the case of government buildings, in' contrast to the findings for commercial of fice buildings, as presented in Tables II-15 and II-16.

It was found that energy consumption varied by building age (Tables III-14 and III-15).: A pattern similar to that found in the case of commercial office buildings emerged.

As was the case with commercial office buildings, government office buildings with computer space tended to use more electricity and less "other fuels", (Tables III-16 and III-17).

Data on energy use in terms of type of air conditioning were developed (Tables III-18 through III-21). Electric air conditioning was present in 164 of the buildings in comparison to steam absorption in 17 buildings.

The data presented in this report should be a useful input to models for the forecasting of energy consumption. However, it should be recognized that the data in this report do not provide a cause and effect explanation of what factors drive energy consumption, nor do the data present energy consumption by end use. A 20 city data base which is being obtained under a different subtask of this project will be subjected to regression analysis under a subsequent task of the project in an effort to obtain insight on what factors cause energy use to vary. No data are gathered for energy use by end use, and it will not be possible, therefore, to develop information on energy use by end use in the regression analysis.

Building energy use simulation programs, such as the DOE II, etc., are typically used to simulate building energy use by end use -- heating, cooling, lighting, hot water, fans, pumps, etc. The programs model energy use as a function of the building shell, the building equipment, and building age. It is currently necessary to rely on these types of programs for end use data, for submetering by. function is still: not practiced to any significant degree in the commercial office building sector. 
The major objective of the Energy Use in Office Buildings subtask presented in this report was to develop the tabular data. As the data were developed two major additional interesting conclusions emerged: there was a decline in office buildings' energy use between 1975 and 1977, based on the comparison of the data in this report with the data from a previous report, and there is a difference in energy use pattern between commercial and government of fice buildings. 


\section{ENERGY USE IN OFFICE BUILDINGS: ANALYSIS OF THE 1977 BOMA DATA BASE}

\section{INTRÓDUCTION}

Energy analysis and projections are generally conducted in terms of four major energy consuming sectors: residential, commercial,' industrial, and transportation. Substantial amounts of detailed data are available for the industrial and transportation sectors; however, data for the residential and commercial sectors have historically been more limited, being available only on a combined basis. The commercial sector is an energy consuming sector which contributes value-added to the GNP. In contrast, the residential sector is an energy consuming sector which consumes the value-added of the GNP. The factors affecting energy consumption by these two sectors are different, for the sectors themselves are different. The work presented in this report is a part of General Electric's efforts over the last 6 years to obtain a better understanding of the factors driving energy consumption in the commercial sector, of which the office building subsector is an important component. This report presents the results of Task IA, Analysis of the 1977 BOMA Data Base, of the Energy Use in Office Buildings Project, work conducted under a cost sharing contract with DOE.

In a previous study, the Commercial Sector Energy Consumption Data Base Development Project, the commercial sector was defined in terms of the activity occurring in 15 major types of space: Store and Other Mercantile Buildings, Warehouses (Excluding Manufacturer Owned), Office and Bank Buildings, Commercial Garages and Auto Service Stations, Schools and College Classroom Buildings, Laboratories (Excluding Manufacturer Owned), Libraries and Museums, Hospitals and Other Health Treatment Buildings, Government Administration Buildings, Other Government Service Buildings, Houses of Worship, Other Religious Buildings, Amusement/Social/Recreational Buildings, Miscellaneous NonResidential Buildings, and Non-Housekeeping Residential Buildings. ${ }^{1)}$ Commercial

1) General Electric Company, Center for Energy Systems, Commercial Sector Energy Consumption Data Base Development Project, DOE Contract EC-77-C-012161. Three major reports resulted from this work: Commercial Buildings Inventory, June 1978; Survey of Available Energy Use Data, April 1978; and Analysis of Energy Consumption in Office Buildings, April 1978. 
sector energy use results from the conduct of economic transactions in a building. The amounts of space, the types of space, the characteristics of the space, and the usage of the space will determine the level of commercial sector energy use. The objectives of the previous contract were the estimation of the inventory of the square feet of 15 types of commercial sector space at the national and regional level, and the assembling of existing information on the energy consumption characteristics of the space. An inventory of commercial space by building type was derived from the F. W. Dodge "Dodge Construction Potentials" for the years 1925-76, and the distribution of the space among the 173 Business Economic Areas (BEA) and building types was derived for 1976. Data for energy consumption by building type were, however, found to be limited: very little information was available for commercial sector energy consumption.

After a broad survey of available information on commercial energy consumption had been conducted, the project focused on energy use in commercial office buildings, which are an important and energy intensive part of the commercial sector. The 1975 data base of the Building Owners and Managers Association International (BOMA) was analyzed. Tables, which present office building energy use as a function of such building characteristics as size, location, height, and age were developed, and a regression analysis presenting energy use as a function of building characteristics was conducted.

The current report presents an analysis of the 1977 BOMA data base. A total of 889 commercial office and 216 government office buildings were analyzed in comparison to the 371 buildings in the 1975 data base. A number of data items, such as information on computer space and air conditioning, which were previously unavailable, are examined. 


\section{THE BOMA ORGANIZATION AND DATA BASE}

The BOMA membership numbers approximately 3800 members in the United States and Canada, with approximately 100 additional members throughout the world. Member buildings account for over 500 million square feet of commercial office space, or about $16 \%$ of total office building space. As the trade association for the commercial office building industry BOMA has a Washington office and staff, and affiliated regional organizations with full or part time staffs are located in approximately 70 U.S. and Canadian cities. The association's work is conducted through a number of committees which deal with such subjects as accounting, energy, marketing, lease content, space measurement, insurance, rehabilitation, planning services, special use buildings, operating methods, urban affairs, etc. The Building Owners and Managers Institute (BOMI) is an affiliated educational organization offering courses in building management; a certification program for the title of Real Property Administrator (RPA) has been developed.

$A$ building manager is responsible on behalf of the owners for the day-to-day operations and maintenance of a building. A typical major commercial office building may be 20 stories tall with 10,000 square feet per floor and may require 75 employees to handle maintenance, renovation, and cleaning. Some managers are also involved in the leasing of the building. Market conditions vary from city to city, but in general newer office space currently rents for $\$ 12-\$ 18$ per square foot per year, and older space is in the $\$ 11-\$ 14$ range. 
BOMA annually collects data on office building characteristics and operating performance. Data are collected from BOMA member and non-member buildings electing to participate in the reporting process; and, in addition, a number of federal and state government buildings have been participating since 1976. Summaries of the data are published on an aggregate basis in the annual Experience Exchange Report; the summaries, which are developed on a city or regional basis provide a benchmark for use by building managers in comparing the results of specific building operations with the industry's aggregate experience. The Experience Exchange Report is a management tool which has been developed to assist building managers in evaluating the cost performance of specific buildings in comparison to overall industry average performance. A copy of the form used by BOMA for data collection is presented in Appendix 1.

The Experience Exchange Report is believed to be the most comprehensive commercial office building data base available; there are, however, some problems associated with it. BOMA undertakes an initial examination of the data for reasonableness, but no extensive verification of the data is performed. In some cases the data are incomplete or inaccurate. In addition, the buildings which participate in the Experience Exchange Report may not be representative of the inventory of all commercial office buildings; the BOMA member buildings are believed to be better maintained and more professionally managed than many commercial office buildings. BOMA has indicated some of the limitations of the data. 1)

"The BOMA Experience Exchange Report is a voluntary report. Consequently, the sample changes from year to year. Moreover, voluntary reporting tends to be less accurate and less complete than mandatory reports. Even though the sample size may be comparable from year to year for a region or city, the building composition of the sample may have changed. For example, the 1978 sample may contain more new buildings than 1977 sample, despite the same number of reports for both years. Consequently, it is important to rely on tables with large samples where possible. Where special circumstances apply, good judgment will be a prerequisite to fruitful use of the BOMA Experience Exchange Report".

1) Building Owners and Managers Association International, 1979 Downtown and Suburban Office Building Experience Exchange Report, Washington, D.C., 1979, p. 3 . 
Access to the 1977 BOMA data base was obtained under a subcontract with BOMA. Data for individual buildings excluded building names and street addresses but were otherwise complete. Under the terms of the agreement, General Electric is precluded from releasing any of the specific individual data items furnished by BOMA. General Electric may publish and/or deliver only aggregated data to the Department of Energy. On completion of such use the data base will be returned to BOMA. The characteristics of the BOMA data base, as reported in Acquisition of The 1977 BOMA Data Base, are presented in Appendix 2. ${ }^{1)}$

Data for 1342 buildings -- 1059 commercial office buildings and 283 government office buildings -- in the United States and Canada were delivered. Of the 1059 commercial office buildings, 999 were located in the United States, and the remainder were Canadian buildings. A total of 233 U.S. Federal, state, and local government operated buildings were also in the data base; 1977 was the first year for which these buildings had participated. Prior to performing the analysis, a major data verification and interpretation effort was conducted by $G E$ in conjunction with the BOMA staff in order to review all relevant data entries and to correct, modify, or delete data items as appropriate and necessary. This effort was necessary because previous work with the 1975 BOMA data base had uncovered many errors and inconsistencies in the energy use and building characteristics data.

For review purposes, electric and fuel use were converted to a per square foot basis, and these data were reviewed along with other building operating and physical characteristics data for reasonableness and accuracy on a building by building basis. ${ }^{2)}$ Questions were referred to the BOMA staff for explanation or correction. In many cases, the BOMA staff were able to verify or correct a data item from existing records. In a number of cases, it was necessary for the BOMA staff to contact individual building managers; in some cases it was impossible for a building manager to verify or correct a data item, and in other cases BOMA could not reach the appropriate person. A number of buildings were, therefore, eliminated from the energy analysis.

1) General Electric, Center for Energy Systems, Acquisition of The 1977 BOMA Data Base, work conducted under DOE purchase order EM-78-X-01-5724, Washington, D.C., December 20, 1978.

2) Electricity was converted at $3412 \mathrm{Btu} / \mathrm{kwh}$; gas at $1020 \mathrm{Btu} / \mathrm{Cu}$. ft.; 非 Oil at 138 , $690 \mathrm{Btu} /$ gallon; 非 6 Oil at 149, $690 \mathrm{Btu} / \mathrm{gallon}$; and Steam at $1000 \mathrm{Btu} / \mathrm{cu}$. $\mathrm{ft}$.

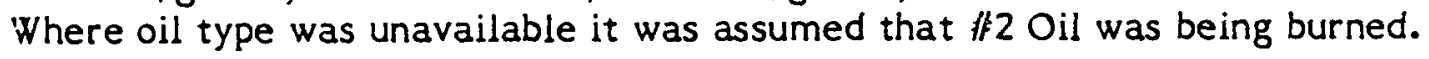


Energy use efficiency varles from bullding to building; however, reports of abnormally high or low energy use were often a result of lnaccurate or incomplete reporting. Where data could be obtained from BOMA the necessary corrections were made. Buildings with inadequate data were not used in the energy analysis. Various factors were found to account for the variability of reported building energy use:

- Special use space: Special use space, such as banking, medical, scientific, retail, restaurant, or corporate headquarters results in energy use that is higher than expected.

- Incomplete energy reporting: In some cases an electric utility directly metered the tenant, and the metered energy use in tenant areas was not reported by the building manager. In other cases, buildings supply. energy to neighboring buildings; this causes reported energy use to be higher than the actual building use for the supplying building. In the case of buildings which purchased chilled water from adjoining buildings, the data were not adequately reported in 1977. Buildings with chilled water usage were dropped from the analysis; for other buildings, the data were either verified or corrected, or the building was dropped.

- Equipment characteristics: Buildings with steam absorption or steam turbine air conditioning have high fuel use but relatively low electric use. Cases with steam absorption and steam turbine air conditioning were identified to the extent possible. The existence of computer space was identified by whether raised flooring and/or special air conditioning was used for the computer equipment. Cases with abnormal electric use were examined to determine whether equipment characteristics could be an explanatory factor for the abnormal energy use.

- Inaccurate reporting: A number of keypunching and data recording errors were found and corrected.

- Fuel use: Cases in which more than one type of fuel use was reported were examined for accuracy. 


\section{The 1977 Data Baser Conclusions}

After the Canadian bulldings and the bulldings with questionable data had been eliminated, a sample consisting of 889 commercial and 216 government office buildings for which data had been verified was finally developed from the data furnished by BOMA. The sample of buildings will be referred to in this report as the "1977 data base", to distinguish it from the somewhat larger data base from which it was obtained, the 1977 Experience Exchange Report. When the 1977 data base is presented in the computer output, it will be denoted as the "1977 BOMA Office Building Sample."

Two analyses were run on the data base: one for the commercial office buildings, and one for the government office buildings. The comments and conclusions for the commercial buildings will be presented in the next section, and the following section will present conclusions for the government buildings. 


\section{ANALYSIS OF THE 1977 DATA BASE: COMMERCIAL OFFICE BUILDINGS}

\section{II-1 Characteristics of the Data Base}

The 1977 data base contained data for 889 commercial office buildings. Before analyzing the data, it was desired to determine to what degree the data were representative of commercial of fice buildings in general. There is a belief that the commercial office buildings from which BOMA collects data are not representative of office buildings in general. Instead, it is believed that BOMA buildings are prime space which is better maintained and managed. However, the 1977 data base does in fact appear to be representative in terms of location and age, on the basis of information obtained from Commercial Buildings Inventery ${ }^{1)}$ Table II-1 compares the distribution of space by census division of the 1977 data base with the distribution of the population of U.S. commercial office buildings in 1975 (there being no 1977 regional data distribution available). ${ }^{2)}$ With the exception of the East South Central region and -- to a lesser degree New England -the 1977 data base is roughly proportionally distributed in comparison to the population of commercial office buildings. In comparison to the 1975 data base developed under a previous contract the 1977 regional distribution is greatly improved. 3 )

1) General Electric Company, Center for Energy Systems, Commercial Buildings Inventory, June 1978.

${ }^{2)}$ The allocation of space by Census Division was based on data in Commercial Buildings Inventory, General Electric, Center for Energy Systems, June.1978.

There are nine census divisions:

New England: Maine, New Hampshire, Vermont, Massachusetts, Rhode Island, Connecticut

Middle Atlantic: New York, New Jersey, Fennsylvania

East North Central: Ohio, Indiana, Illinois, Michigan, Wisconsin

West North Central:

Minnesota, Iowa, Missouri, North Dakota, South Dakota, Nebraska, Kansas

South Atlantic:

Delaware, Maryland, District of Columbia, Virginia, West

East South Central: Virginia, North Carolina, South Carolina, Georgia, Florida

West South Central: Kentucky, Tennessee, Alabama, Mississippi

Mountain:

Arkansas, Louisiana, Oklahoma, Texas

Montana, Idaho, Wyoming, Colorado, New Mexico, Arizona, Utah, Nevada

Pacific: Washington, Oregon, California, Alaska, Hawaii.

3)

Throughout this report reference will be made to the 1975 data base, which is presented in Analysis of Energy Consumption in Office Buildings, General Electric Company, Center for Energy Systems, April 1978. 
TABLE II-1.

PERCENTAGE DISTRIBUTION OF SQUARE FEET OF COMMERCIAL OFFICE BUILDING SPACE BY CENSUS DIVISION

Estimated Distribution

of U.S. Commercial Offige
Building Space for .1975

(Percent of Total Space

Census Division
Distribution of Square

Feet of Commercial Space in 1977 Sample

(Percent of Sample)
Distribution of Square

Feet of Commercial 3 ) Space in 1975 Sample .. (Percent of Sample)

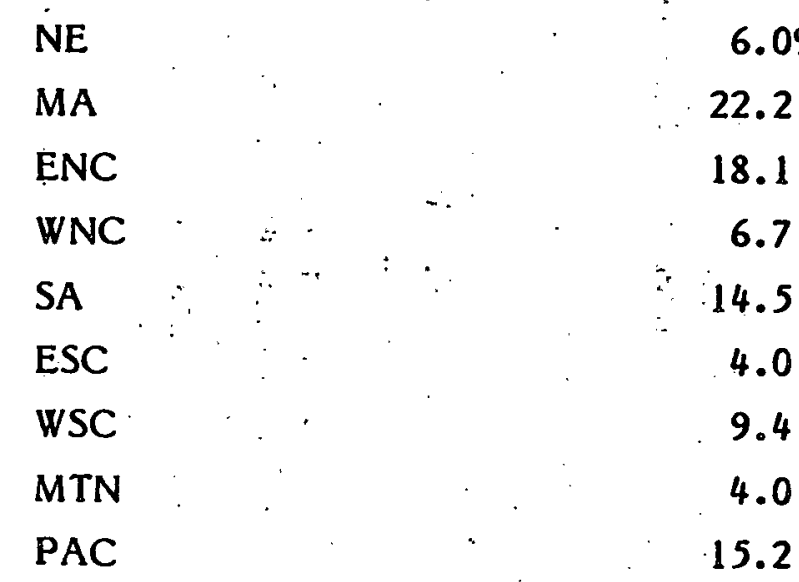

$2.8 \%$

21.6

15.6

8.8

13.4

1.1 .

14.4

4.8

17.6
$.7 \%$

33.7

19.0

9.6

5.5

.3

13.8

1.9

15.5

1) Based on Commercial Buildings Inventory of the Commercial Sector Energy Consumption Data Base Development Project, DOE Contract EC-77-01-2161, Center for Energy Systems, General Electric Company, June 1978.

2) Based on the data base developed for this project.

3) Based on the data base presented in Analysis of Energy Consumption in Office Buildings of the Commercial Sector Energy Consumption Data Base Development Project, DOE Con tract EC-77-C-01-2161, Center for Energy Systems, General Electric Company, April 1978. 
Table II-2 presents the distribution at the national level of commercial office building space by age as developed from data presented in Commercial Building Inventory and compares both the 1977 and the 1975 data bases. With the exception of buildings aged 30-39 years, the 1977 data base is an improvement over the 1975 data base and is not unrepresentative of the population of buildings in general.

The buildings in the 1977 data base can be defined in terms of the type of fuel used for heating: electric, gas, oil, and steam.

\begin{tabular}{lcccc}
$\begin{array}{c}\text { Building Fuel } \\
\text { Type }\end{array}$ & $\begin{array}{c}\text { Number of } \\
\text { Buildings }\end{array}$ & $\begin{array}{c}\text { \% of } \\
\text { Buildings }\end{array}$ & $\begin{array}{c}\text { Square Feet } \\
\text { of Spgce } \\
\left(10^{5}\right)\end{array}$ & $\begin{array}{c}\% \text { of } \\
\text { Space }\end{array}$ \\
\hline Electric & 172 & $19.4 \%$ & 34.2 & $15.3 \%$ \\
Gas & 359 & 40.4 & 78.9 & 35.4 \\
Oil & 71 & 8.0 & 16.0 & 7.2 \\
Steam & $\frac{787}{889}$ & 32.3 & 94.0 & 42.1
\end{tabular}

Table II-3 describes the buildings by fuel type and census division. Approximately $40 \%$ of the electrically heated buildings are centered in the South Atlantic division with an additional $26 \%$ in the Pacific. Gas heated buildings are relatively evenly distributed throughout the country. The major concentration of oil heated buildings, which are relatively few in number, is in the South Atlantic Division. There are four major concentrations of steam heated buildings: Middle Atlantic, East North Central, West North Central, and Pacific. Steam heated buildings are concentrated in major cities with steam plants.

Most of the buildings for which data are reported in the BOMA Experience Exchange Report are probably prime, well managed space; it is unlikely that data are reported for non-prime space. Since there is no information available at the national level for the mix of prime and non-prime space, however, it is not possible to estimate to what degree the 1977 data base is unrepresentative of buildings in general. 
TABLE II-2

" PERCENTAGE DISTRIBUTION OF SQUARE FEET OF COMMERCIAL OFFICE BUILDING SPACE BY AGE :

Estimated Distribution of Square Feet of Commercial Office

Building Space by Age in 1977

Age

(Percent of Total Space)
Distribution of Square

Feet of Commercial Office Building Space $)^{\text {in }} 1977$

(Percent of Sample)
Distribution of Square . Feet of Commercial Office Building Space in 1975 Sample
$1-9$

$10-19$

20-29

30-39

$\varpi$
$47.1 \%$
24.6
9.5
4.4
4.9
9.5

$\begin{array}{cc}45.7 \% & 42.0 \% \\ 21.8 & 22.5 \\ 7.8 & 4.9 \\ .1 & 4.0 \\ 9.6 & 12.0 \\ 14.0 & 14.6\end{array}$

1) Estimated on the basis of data in Commercial Buildings Inventory of the Commercial Sector Energy Consumption Data Base Development Project, DOE Coritract EC-77-C-01-2161, Center for Energy Systems, General Electric Company, June 1978. Based on yearly additions adjusted by removal rate.

2) Based on data developed in this project.

3)

Based on data presented in Analysis of Energy Consumption in Office Buildings of the Commercial Sector Energy Consumption Data Base Development Project, DOE Contract EC-77-C-01-2161. 
TABLE II-3

THE 1977 BOIMA OFFICE BUILDING SAMPLE

BY TYPE OF. HEATING. FUEL AND REGION

(SOUARE FEET IN MILLIONS)

COMMERCIAL OFFICE BUILDIHGS

ELECTRIC

NUMBER SO. SOFT BLDG

OF BLDGS
GAS

NUMBER SO. SOFT/BLDO OF BLDGS
OIL

NUMBER SO. SOFT/BLDG OF. BLDGS FT. (OOO)

0.0

NEW ENGLAND

MIDELE ATLANTIC

EAST NURTH CENTRAL

$0 \quad 0.0$

3

0.6

213.1

4.0

16

61

43

43. $7.1 \quad 166.2$

SOUTH ATLANTIC

EAST SOUTH CENTRAL

WEST SUUTH CENTRAL

MOUNTAIN

PACIFIC

TOTAL il. $2.3 \cdot 206.4$

$\begin{array}{lll}3 & 0.7 \quad 224.1\end{array}$

$\begin{array}{lll}22 & 4.9 & 222.9\end{array}$

$6 \quad 1.3 \quad 222.6$

$\begin{array}{lll}.45 & 8.4 & 185.8\end{array}$

$-$

172

34.2

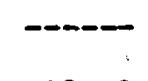

$\begin{array}{lll}68 & 11.4 & 167.5\end{array}$

$\begin{array}{llll}38 & 3.9 & 103.2\end{array}$

$5: 0.6 \quad 120.6$

$84 \quad 25.2 \quad 299.9$

4. $\quad \begin{array}{lll}0.0 & 1.47 .1\end{array}$

$\begin{array}{llll}68 & 17.0 \quad 250.0\end{array}$

$-$

.359

$78.9 \quad 219.9$

\begin{tabular}{ccc}
2 & 1.0 & 488.5 \\
6 & 0.9 & 143.1 \\
6 & 2.7 & 452.1 \\
4 & 0.6 & 157.3 \\
49 & 9.8 & 199.4 \\
1 & 0.7 & 737.2 \\
1 & 0.3 & 286.2 \\
0 & 0.0 & 0.0 \\
2 & 0.1 & 29.7 \\
- & -2.0 & $-.7-$ \\
\hline 71 & 16.0 & 225.8 \\
\hline
\end{tabular}

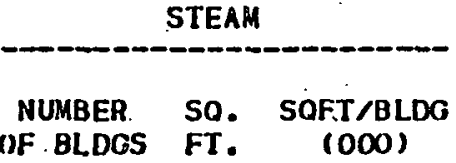

. $54.6: 923.2$

$84 \quad 44.8 \quad 532.8$

$\begin{array}{lll}52 & 10.8 \quad 207.4\end{array}$

$\begin{array}{lll}42 & 9.6 & 229.4\end{array}$

$14 \quad 4.9 \quad 346.5$

$\begin{array}{lll}4 & 0.5 & 126.0\end{array}$

$2 \quad 1.8 \quad 875.5$

i4 $\quad 3.3 \quad 238.7$

$70 \cdot 13.8 \quad 1.97 .1$

- - - - -

$\begin{array}{lll}287 & 94.0 \quad 327.7\end{array}$ 
TABLE II-4

HEATING FUEL BY AGE OF BUILDING IN THE 1977 BOMMA OFFICE BUILDING SAMPLE. (SOUARE FEET IN MILLIONS)

COAMERCIAL OFFICE BUILOINGS

HEATING FUEL

\begin{tabular}{|c|c|c|c|c|c|c|c|c|c|c|c|c|c|c|c|c|}
\hline & & \multicolumn{3}{|c|}{ ELECTRIC } & \multicolumn{3}{|c|}{ GAS } & \multicolumn{3}{|c|}{$01 \mathrm{~L}$} & \multicolumn{3}{|c|}{ STEAM } & \multicolumn{3}{|c|}{ TUTAL } \\
\hline & $\begin{array}{c}\text { AGE } \\
\text { (YEARS) }\end{array}$ & $\begin{array}{l}\text { No. (IF } \\
\text { BLDGS }\end{array}$ & $\begin{array}{l}\text { So. } \\
\text { FT. }\end{array}$ & $\begin{array}{l}\text { SO.FT./ } \\
\text { BLDG } \\
(000)\end{array}$ & $\begin{array}{l}\text { No. IIF } \\
\text { BLDGS }\end{array}$ & $\begin{array}{l}\text { So. } \\
\text { FT. }\end{array}$ & $\begin{array}{l}\text { SO.FT.' } \\
\text { BLDG } \\
(000)\end{array}$ & $\begin{array}{l}\text { No.of } \\
\text { BLDSS }\end{array}$ & $\begin{array}{l}\text { So. } \\
\text { FT. }\end{array}$ & $\begin{array}{c}\text { SO.FT.' } \\
\text { BLDG } \\
\text { (000) }\end{array}$ & $\begin{array}{l}\text { N(). (OF } \\
\text { BLDGS }\end{array}$ & $\begin{array}{l}\text { SO. } \\
\text { FT. }\end{array}$ & $\begin{array}{l}\text { SO.FT./ } \\
\text { BLDG } \\
(000)\end{array}$ & $\begin{array}{l}\text { No.()F } \\
\text { BLDGS }\end{array}$ & $\begin{array}{l}\text { So. } \\
\text { FT. }\end{array}$ & $\begin{array}{l}\text { SO.FT./ } \\
\text { BLDG } \\
\text { (000) }\end{array}$ \\
\hline & $1-9$ & 122 & 27.9 & 228.3 & 121 & 33.7 & 278.7 & 26 & 7.3 & 280.7 & 53 & 33.2 & 626.5 & 322 & 102.1 & 317.0 \\
\hline & $10-19$ & $2 A$ & 3.4 & 143.4 & 98 & 18.6 & 190.2 & 23 & 5.0 & 218.6 & 60 & 21.6 & 360.3 & 205 & 48.7 & 237.7 \\
\hline & $20-29$ & 5 & 1.0 & 190.1 & 43 & 9.2 & .215 .1 . & 8 & 1.5 & 190.7 & 19 & 5.7 & 300.6 & 75 & 17.4 & 232.5 \\
\hline . & $30-39$ & 1 & 0.0 & 9.7 & 8 & 2.2 & 26.9 .6 & 2 & 0.1 & 33.0 & 0 & 0.0 & 0.0 & 11 & 2.2 & 203.0 \\
\hline 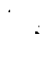 & $\begin{array}{c}40-4.9 \\
\therefore\end{array}$ & 4 & 0.7 & 187.0 & 16 & 3.6 & $226 . .1$ & 3 & 1.4 & 470.3 & 43 & 15.6 & 363.3 & 66 & 21.4 & 324.2 \\
\hline & $50+$ & 16 & 1.2 & 74.9 & 73 & 1.1 .5 & .158 .2 & 9 & 0.7 & 78.4. & 112 & $17.9^{\circ}$ & 159.7 & 2.10 & 31.3 & 149.2 \\
\hline & TOTAL & 172 & 34.2 & 198.9 & 359. & 78.9 & 219.9 & 7.1 & 16.0 & 22.5 .8 & 287 & 94.0 & 327.7 & 889 & 223.2 & 251.1 \\
\hline
\end{tabular}


Table II-4 presents data on fuel type and building age. $71 \%$ of the electrically heated buildings are less than 10 years old. In many areas natural gas has been unavailable or subject to uncertain supply for a number of years. Depending on climate, expected usage, and capital costs, it has been cheaper in some instances to rely on electricity than to install an oil/gas dual fuel capability. Installation of electric heating also eliminates problems of fuel availability. Natural gas, however, has continued to be available to some degree: $34 \%$ of the natural gas heated buildings are less than 10 years old. Most of the oil heated buildings are less than 20 years old. During the 1960's and early 1970's oil was cheaper than natural gas and captured a significant share of the market in some cities. The steam heated buildings tend to be older than buildings heated by other fuels. District steam plants have existed for many years in some of the large cities.

\section{II-2 Summary of Energy Consumption}

Energy consumption in terms of Btu/Sq. Ft. was prepared on a Census Division basis for all fuels together and for electric, gas, oil, and steam buildings separately. The tables - Tables II-5 through II-12 -- are presented at the end of this section. Btu/Sq. Ft. was calculated on a weighted and unweighted basis for electric use, fuel use, and total energy use. A computation with the Btu/Sq. Ft. for each building weighted by the area of the specific building relative to the total sample places a greater importance on the larger buildings, which are the important ones in terms of the total square feet of building space. In performing an unweighted computation, all buildings are assumed to be of equal importance regardless of size. It is believed that the weighted means and variances are the more important statistics, and the discussions throughout this report will focus on them. Unweighted means and variances will, however, in a number of instances also be presented.

In cases in which both weighted and unweighted means and variances have been prepared the weighted data will be presented in a table with the suffix $A$. The unweighted data will be presented immediately following in an identically numbered table with the suffix $B$. 
It will be observed that the means of "Electricity" and "Other" fuels in general do not exactly add to equal the "Total" energy use in many of the following tables. The differences are relatively minor and are due to the way in which the data were processed and computed.

1. In the case of electrically heated buildings, the number of buildings in the denominator for "Other" fuel use does not equal the total number of electrically heated buildings. "Other" fuel was computed only for those buildings which in fact had such use.

2. In the case of gas, oil, and steam heated buildings the "Other" fuels heading captures the use of the principal fuel -- gas, oil, or steam -- not total fuel use. However, total fuel use was included in the "Total" energy use column. For example, many oil and steam heated buildings have minor amounts of gas use for restaurant or other purposes, this use was included in the "Total" column but not in the fuel use column. Similarly, some gas heated buildings had significant amounts of oil use -- probably due to interruptible gas supplies. This oil use was included in the "Total" heading but not under the "Other" fuel heading.

3. The number of buildings in the denominator was not always equal in computing "Electricity", "Other" fuel use, and "Total" use. For example, for a few buildings either "Other" or "Electricity" were not reported in the data base, but "Total" energy use was available. In these cases, rather than eliminate a building entirely, the building was only eliminated from that part of the computation for which the data were unavailable. It is, therefore, necessary to note that Table II-3 does not present the exact number of buildings in each computation. Rather, Table II -3 is most useful in examining fuel penetration. The number of buildings in each computation is explicitly presented in Table II-7.

In presenting the results developed from the 1977 data base we will make some comparisons with the 1975 data base, developed under a previous contract. It should be recognized that the 1975 sample was not as large or as well verified as the 1977 sample. For example, in the case of the 1975 data outlying observation - i.e., energy use observations which appeared to $G . E_{0}$ to be questionable -- were more often eliminated without additional review or correction by BOMA than was the case with the 1977 data base. This probably did not bias the mean, but it 
almost certainly caused the computed sample variance to be less than the population variance. As a result, the 1975 data base had significantly fewer buildings, and the data tended to be more clustered about the means. In addition to differences in sample size and possible bias, the regional distribution of the 1975 sample was not as good as that of the 1977 sample.

Table Il-5 presents a summary of energy use by type of fuel at the national level during 1975 and 1977. In non-electrically heated buildings the fuel is in general used for building heating and hot water; however, a limited amount of gas and steam air conditioning also accounts for some of the fuel use. The electricity is used for the usual purposes of lighting, fans, etc.; and in most buildings the air conditioning is electric. It would be desirable to have a finer breakout by end use - lighting, heating, hot water, air conditioning, fans, pumps, special equipment, etc. However, most buildings do not meter energy use in this way: the data are, therefore, unavailable. To determine energy use by end use it is currently necessary to use a building simulation program, such as the AXCESS program.

Recognizing that there are limitations to the comparison of 1975 and 1977 data it is, nevertheless, useful to examine the differences between the two years. Total Btu/Sq. Ft. was 152.5 thousand in 1977 in comparison to 159.9 thousand in 1975. With the exception of steam heated buildings commercial office buildings used less energy in 1977 than in 1975.

It was desired to determine on a statistical basis whether energy use per square foot had changed between 1975 and 1977. Recognizing that the computed 1975 standard deviation was unreliable we formed $95 \%$ and $99 \%$ confidence intervals for the 1977 population mean based on the sample, as is shown in Table II6. On a total energy use basis the 1975 sample mean did not fall in the 1977 confidence interval for all buildings on a combined basis and for electric, gas, and oil fired buildings. Accordingly, the statement that conservation occurred between 1975 and 1977 is not disproved. 
Electrically heated buildings used the least energy per square foot in 1977 in comparison to other types of buildings. Building location may have been an important contributing factor to this difference, for most electric buildings are located in relatively warm climates; therefore, heating requirements would be less than would be the case for other building types. In addition, in the case of electric buildings, energy is measured at the user level, exclusive of generation and transmission losses. There was a substantial decline in Btu/Sq. Ft. use by the electrically heated buildings between 1975 and 1977.

Steam heated buildings were the second most energy efficient in 1977, but 1977 total Btu use was up by approximately nine thousand Btu/Sq. Ft. between 1975 and 1977. As can be seen in Table II-5, the increase was entirely caused by rising electric use, probably due to increased supplemental use of electricity in place of steam for some heating applications. As is the case with electricity, steam use is measured at the building line exclusive of generation and transmission losses: energy use data are measured in this report on the basis of building expenditures. The place of measurement has an effect on the apparent efficiency of utilization: in the case of electric and steam buildings, much of the energy loss occurs before the energy is measured in the tables; in the case of gas and oil use, the tables pick up much of the energy utilization loss.

Gas heated buildings were the least energy efficient, probably due in part to the relatively low cost of gas. Even here, however, there was a decline of twenty thousand Btu/Sq. Ft.

Table II-7 summarizes fuel use on a weighted basis by building type and census division. Tables II-8 through II-12 present the detailed computer output.

Both electricity and fuel use vary by region. For gas, oil, and steam heated buildings less electricity is in general used in colder than in warmer areas of the country, probably as a result of the decreased need for air conditioning. Conversely, more fuel is used in colder areas of the country, probably resulting from the increased need for heating. For electrically heated buildings electric use also appears to vary by climate area, but it is not possible to distinguish between fuel use and air conditioning use. The "other" energy use for electrically heated buildings is largely fuel used for restaurant, decorative, or other process use purposes. 
TABLE II-5

ENERGY CONSUMPTION IN THE 1975 AND 1977 COMMERCIAL OFFICE BUILDING SAMPLES BY TYPE OF HEATING FUEL

(Btu/Sq. Ft. in thousands; Square feet in millions) Weighted Means

\begin{tabular}{|c|c|c|c|c|}
\hline \multicolumn{4}{|c|}{ Heating Fuel } & All Buildings \\
\hline Electric & Gas & Oil & Steam & \\
\hline 1977 & 1975 & 1977 & 1975 & 1975 \\
\hline
\end{tabular}

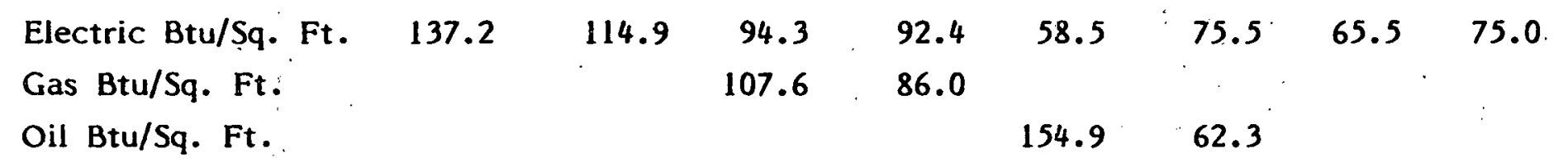

$\infty_{\infty} \quad \cdots \quad$ Steam Btu/Sq. Ft. Total Btu/Sq. Ft. 1)

Total Square Feet

Number of Buildings

$\begin{array}{rrrrrrrrrr}137.2 & 116.6 & 201.9 & 180.1 & 213.4 & 152.9 & 132.6 & 141.4 & 159.9 & 152.5 \\ 19.7 & 34.2 & 39.4 & 78.9 & 12.2 & 16.0 & 68 & 94 & 139.3 & 223.1 \\ 41 & 158 & 139 & 333 & 30 & 65 & 161 & 273 & 371 & 829\end{array}$

1) As is indicated in the text, electric use plus other fuel use do not exactly sum to equal total energy use. 


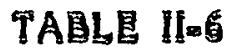

CONEIDENCE INTERVALS BOR PHE 1977 MEAN

POR COMMERCIAL OPIICE RUILDINGS

(Btu/SG. Pet. In thousands)

\section{Ruel Type}

Eleetric

939. cenfldence Interva! 99\% centidenee Interva!

Gas

5

959 cenfldence Interva! 99 centldence Interval

Oll

95\% confidence Interva! 99\% contldence Interva!

\section{Steam}

95\% confldenee Interva! $99 \%$ confldence Interval

All Gulldings

95\% cenfldence Interva! 99\% confldenee Interva!
1977 Mean

116.6

$111.0-122.2$

$109.3=123.9$

180.1

201.9

$167.7=192.5$

163.8 .196 .4

152.9

213.4

138.29167 .6

133.5172 .3

141.4

132.6

$135.6 \times 147.2$

$133.8=149.0$

1928

159.9

146.9 .158 .0 143.20159 .8 
TABLE II-7

SUMMARY OF 1977 COMMERCIAL OFFICE BUILDING ENERGY CONSUMPTION ${ }^{1)}$

( $10^{3}$ BTU/SQ. FT.)

WEIGHTED MEANS

- Census Division and.Type of Building Fuel for Heating

Ejectricity

Number of Buildings

Other

Number of

Buildings

Tota!

Number - of

New England

Electric

Gas

Oil

All

Middle Atlantic

Electric

- Gas

Steam

ast North Central

Electric

Gas
Oil

Steam

All.

West North Central Electric

Gas

Stẹam

Ali

- South Atlantic

Electric

Gas

Steam

$$
\text { Ail }
$$

East South Central Electric.

Gas. 2

Steam

Al!

West South Central Electric.

Gas
Oil

Steam

All

67.9
73.7
64.2
66.1

0
3
2
5
10

97.3
104.5
103.7

103.7
103.2

0
3
2
5
10

165.1

167.9
169.3

181.7

104.1

54.3
76.2
77.3

1

105.7

105.7
78.6

78.6
70.4

00181.7

$77.3 \quad . \quad .97$

117.5

65.6

60.5
77.7

76.8

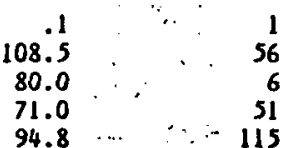

197.0
151.5

146.8

146.8
149.4

14
56
6
$5 i$
128

128.6
84.8
64.0

64.0
67.9

81.2

$$
\begin{array}{r}
11 \\
37 \\
3 \\
42 \\
97
\end{array}
$$

3.5
116.7

116.7
42.9
66.1

96.1

106.0
82.0

82.0
78.8

78.8
75.3
88.6

62

62
35

35
46
13

102.7

102.7
96.7

96.7
126.0

100.2

109.1

13
159

119.0

$85 . \overline{5}$

114.2

3
4
1
4
12
19
19
80
0
2
102

9.0
73.3

53.7

68.4
61.0

61.0

4.4
58.8
48.5

48.5
38.7
70.1

70.1

5
75
96

117.5

183.0

168.3

151.1
162.4

Buildings

1) See footnote on next page. 
TABLE II-7, Continued

SUMMARY OF 1977 COMMERCIAL OFFICE BUILDING ENERGY CONSUMPTION ${ }^{1)}$

$\left(10^{3}\right.$ BTU/SQ. FT. $)$

WEIGHTED MEANS

Census Division

and Type of Building

Fuel for Heating

Number of

Buildings

Mountain

Electric

Gas

Steam

All

Pacific

Electric

Gas

AII

National Average

Electric

Gas

Steam

$\begin{array}{rr}132.4 & 5 \\ 85.4 & 38 \\ 86.5 & 0 \\ 92.3 & 13 \\ & 58\end{array}$

117.7

37.6

2.4

43
64

66
179

114.9

92.4

75.5

75.0

. 158

158
331

331
65

65
273

All

Other

Number of

Total

Number of

Buildings

28.8

5.8
122.7
53.6
93.3

28.8
48.6
67.6
37.8

43.3

Buildings

842

9.1
86.0
62.3
66.2
74.9

9.1
86.0

74.9

1
38
0
13
53

135.9

208.8

141.0

179.8

5
38
0
13
58

121.8

134.5

105.3

109.5
123.1

43

62
2
65

138

16.6

- 20

116.6

180.1

152.9

141.4
152.5

64
269

700

. 158

333

65
273

273
844

1) As is indicated in the.text, "Electricity" plus "Other" may not exactly equal "Total" energy use. Similarly, the numbers of electric, gas; oil, and steam buildings do not exactly sum to equal the number of. "All" buildings, for some buildings were not included in some computations. 
TABLE [I - $8 A$

ENEROY CONSUMPTIUN IN THE 1977 B(I)MA (IFFICE BUILDINO SAMPLE (BTU/SQ.FT: 000 )

WEIGHTED MEANS AND STANDARD DEVIATIONS COMAERCIAL OFFICE BUILOINGS

ELECTH ICITY

MEAN STD.DEV.

NEW ENGLAND

MI DOLE ATLANTIC

EAST NURTH, CENTRAL

WEST. NISRTH CENTRAL

SOUTH ATLANTIC

EAST SUUTTH CENTRAL

WEST SOUTH CENTRAL.

MOUNTAIN

PACIFIC

NATIONAL AVERAGE

$$
66.1
$$$$
14.0
$$

77.3

20.0

76.8

32.3

81.2

34.1

88.6

36.3

109.1

.22 .3

11.4 .2

48.2

92.3 .

34.5

87.9

41.7

87.0

38.2
MEAN STD.DEV.

$103.2 \quad 14 . \dot{4}$

72.2

36.4

94.8

58.2

93.2

96.1

6.1 .0

59.1

70.1

39.4

$77.6: 1.25 .7$

$93.3 \quad 1.12 .4$

43.3

.43 .3

7.4 .9

75.2
TUTAL

MEAN STD.DEV:

$169.3 \quad 16.4$

$149.4: 41.7$

162.461 .1

164.1 .89 .8

$131.0 \quad 63.6$

$161.4 \quad 57.5$

$180.6 \quad 137.8$

$179.8 \quad 118.4$

.1 .23 .156 .4

$152.5 \quad 81.6$

TABLE II - 8B

ENERGY CINSUMPTION IN THE 1977 BUMA OFFICE BUILDING SAMPLE

(BTU/SO.FT. OOO) UNWEIGHTED MEANS AND STANDARD DEVIATIONS COMMERCIAL OFFICE BUILDINGS

ELECTRICITY

MEAN STD.DEV.

OTHER FUELS

TOTAL

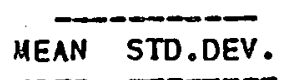

MEAN STD.DEV.

NEW ENGLAND

66.5

13.4

$103.9 \quad 16.0$

170.3

17.6

\section{MIDDLE ATLANIIS}

75.5

31.8

70.85. 35.9

145.3

41.3

EAST NORTH CENTRAL

$72.3 \quad 35.1$

94.8 .56 .8

157.4

65.8

WEST NORTH CENTRAL

$79.9 \quad 35.7$

$94.3 \quad 73.5$

163.1

75.7

SOUTH ATLANTIC

$.85 .9 \quad 34.7$

$64.2 \quad 59.9$

$1.28 .7 \quad 59.3$

EAST SUUTH CENTRAL.

$97.3 \quad 3.1 .4$

$51.3 \quad 39.5$

140.1

49.5

WEST SOUTH CENTRAL

$106.4 \quad 48.1$

76.0

114.6

168.3

122.7

MUUNTAIN

78.9

36.3

$90.4 \quad 97.3$

16.1.5. $\quad 104.2$

PACIFIC

76.9

45.2

47.9

45.1

113.9

60.9

NATIONAL AVERAGE

$82.0 \quad 39.9$

74.5

70.0

143.6

77.0 
TABLE II - 9 A

ENEROY CONSUMPTION IN THE 1977 BOMA OFFICE BUILDING SAMPLE

ELECTR ICALLY HEATED BUILDINOS

WEIGHTED MEANS AND. STANDARD DEVIATIUNS

COMMERCIAL. OFFICE BUILDINGS

ELECTRICITY

MEAN STD.DEV. MEAN STD.DEV.

MEAN STD.DEV.

NEW ENOLAND

0.0

0.0

$0: 0$

0.0

0.0

MIDOLE ATLANTIC

181.7 Undefined 1$)$

0.0

0.0

18..7 Undefined 1 )

EAST NORTH CENTRAL.

$117.5 \quad 28.3$

0.1 Undefined ${ }^{\text {l) }}$

$117.5 \quad 28.3$

WEST NORTH CENTRAL

$128.6 \quad 28.5$

3.5 Undefined

$129.4 \cdot 28.1$

SUUTH ATLANTIC

$106.0 \quad 35.4$

$9.0 \quad 10.5^{22}$

107.9

35.1

EAST SIUTH CENTRAL

102.7

9.3

4.4 Undefinedl)

103.4

9.4

WEST SOUTH CENTRAL

119.0

41.8

0.0

0.0

119.0

41.8

MOUNTAIN

132.4 .56 .3

5.8 Undefined 1 )

135.8

54.8

PACIFIC

$117.7 \quad 31.8$

$28.824 .3^{2)}$

121.8

31.7

NATIONAL AVERAGE

$11.4 .9 \quad 35.9$

9.1

14.9

116.6

35.7

1) One building.

2) Mean and standard deviation computed only for those bulldings having "other" use.

TABLE II - 98

ENERGY CONSUMPTION IN THE 1977 BOMA OFFICE BUILDING SAMPLE ELECTRICALLY HEATED BUILOINSS

(BTU/SO.FT, OOO)

UNWEIGHTED MEANS AND STANDARD DEV IAT IONS

COMHERCIAL OFFICE BUILDINGS

ELECTRICITY

MEAN STD.DEV.

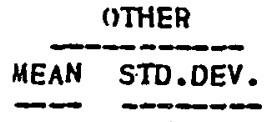

MEAN $\frac{\text { TOTAL }}{\text { STD.DEV }}$

NEW ENGLAND

0.0

0.0

0.0

0.0

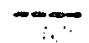

STD.DEV.

MI DDLE ATLANTIC

181.7

0.0

0.0

0.0

0.0

0.0

EAST NURTH CENTRAL

$111.1 \cdot 46.3$

0.1

0.0

181.7

0.0

WEST MORTH CENTRAL

$121.8 \quad 35.7$

3.5

$0.0^{11}$

111.1

.46 .3

SUUTH ATLANTIC

$102.9 \quad 32.7$

8.6

$10.5^{1)}$

122.1

35.6

EAST SOUTH CENTRAL

$102.5 \quad 10.2$

4.4

0.0

.104 .6

32.8

WEST SIUTH CENTRAL

120.0

48.7

0.0

0.0

104.0

10.5

MOUNTAIN

131.5

77.5

5.8

0.0

120.0

48.7

PACIFIC

102.3

40.8

19.6

$25.41)$

132.6

77.1

NATIONAL AVERAGE

108.2

40.6

9.7

$1.4 .0^{1)}$

.104 .1

41.6

109.5

40.7

1) Mean and standard deviation of "other" computed only for those buildings having "other" use. 
TABLE II $-10 A$

- ENERGY CONSUAPTION IN THE 1977 BUMA OFFICE BUILDING SAMPLE GAS HEATED BUILDINGS

(BTU/SO.FT. OOO)

WEIGHTED MEANS. AND STANDARD DEVIATIONS COMMERCIAL OFFICE BUILDINGS

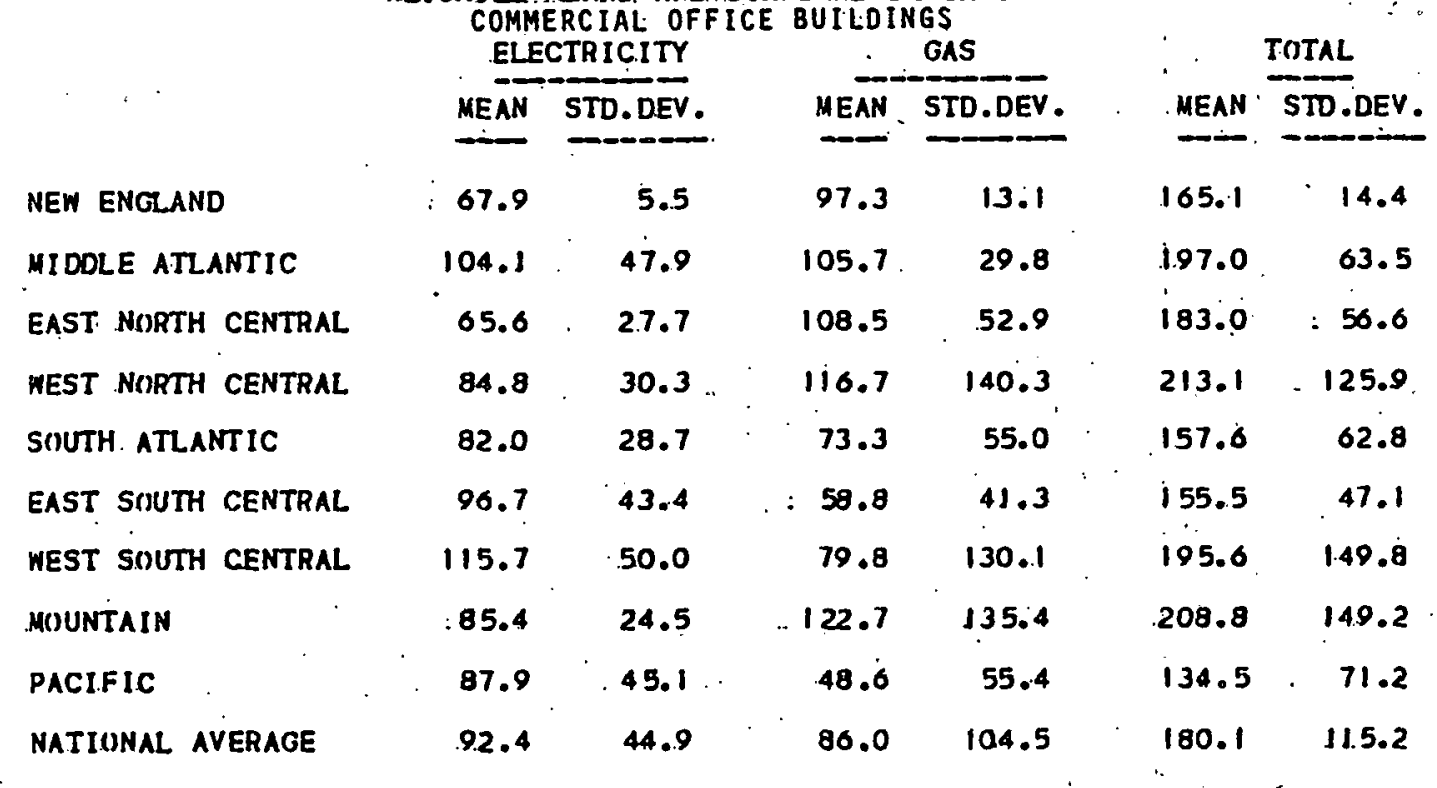

TABLE II - 10B

ENERGY CONSUMPTION IN THE 1977 BOMA OFFICE BUILDING SAMPLE. GAS HEATED BUILDINGS

(BTU/SO.FT, OOO) UNWEIOHTED MEANS AND STANDARD DEVIATIONS COMMERCIAL OFF ICE BUILDINGS

ELECIR I CITY

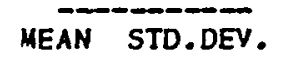

NEW ENGLAND

MIDOLE ATLANTIC

EAST NORTH CENTRAL

WEST NORTH CENTRAL

SOUTH ATLANTIC

EAST SOUTH CENTRAL

WEST SOUTH CENTRAL

MOUNTAIN

PACIFIC

NATIONAL AVERAGE

\begin{tabular}{lllll}
\multicolumn{1}{c}{ GAS } & & \multicolumn{2}{c}{ TOTAL } \\
\hline MEAN & STD.DEV & & MEAN & \multicolumn{1}{l}{ STD.DEV } \\
\hline 97.3 & 16.3 & 165.3 & 17.9 \\
98.8 & 39.9 & 169.5 & 03.4 \\
107.0 & 50.1 & 175.6 & 57.5 \\
.95 .8 & 97.1 & 187.9 & 97.9 \\
76.3 & 63.6 & 149.7 & 67.5 \\
60.4 & 48.7 & 150.3 & 56.8 \\
77.7 & 116.3 & 182.1 & 133.2 \\
100.4 & 109.0 & 173.4 & 119.9 \\
52.7 & 61.5 & 123.3 & 83.9 \\
83.4 & 87.5 & 164.9 & 99.9
\end{tabular}


TABLE II - IIA

ENERGY CONSUMPTION IN THE 1977 BOMA OFFICE BUILDING SAMPLE

(IIL HEATED BUILDINOS

(BTU/SQ.FT. OOO)

WEIGHTED MEANS AND STANDARD DEVIATIUNS

COMMERC.IAL OFFICE BUILDINGS

- ELECTRICITY

MEAN STD.DEV.

()IL.

TUTAL

\begin{tabular}{|c|c|c|c|c|c|c|}
\hline 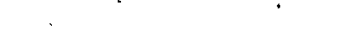 & MEAN & STD.DEV. & MEAN & STD.DEV. & MEAN & STD.DEV. \\
\hline NEW ENGLAND & 73.7 & 0.2 & 104.5 & 14.7 & 178.1 & $i 14.4$ \\
\hline MI DOLE ATLANTIC: & 54.3 & $19 . .9$ & 78.6 & 10.8 & 151.5 & 13.7 \\
\hline EAST NORTH CENTRAL & 60.5 & 16.4 & 80.0 & 47.5 & 168.3 & 7.4 .6 \\
\hline WEST NORTH CENTRAL & 64.0 & 18.9 & 42.9 & $14.8 \ldots$ & 143.8 & 30.3 \\
\hline SOUTH ATLANTIC & 78.8 & 37.0 & 53.7 & 47.0 & 140.9 & 58.9 \\
\hline EAST SOUTH CENTRAL & 1.26 .0 & Undefined ${ }^{1)}$ & .48 .5 & Undefinedl) & 231.2 & Undeftned ${ }^{1}$ \\
\hline WEST SOUTH CENTRAL & 0.0 & 0.0 & 0.0 & 0.0 & 0.0 & 0.0 \\
\hline MOUNTAIN & 0.0 & 0.0 & 0.0 & 0.0 & 0.0 & 0.0 \\
\hline PACIFIC & 37.6 & 2.0 & 67.6 & 12.6 & 105.3 & 14.6 \\
\hline NATIONAL AVERAGE & 75.5 & 33.7 & 62.3 & $45.2 \cdot$ & .152 .9 & 60.5 \\
\hline
\end{tabular}

TABLE II - $11 B$

ENERGY CONSUMPTION IN THE 1.977 BOMA IFFICE BUILDING SAMPLE OIL HEATED BUILDINGS

(BTU/SO.FT. OOO)

UNWEIGHTED MEANS AND STANDARD DEVIATIONS

COMMERCIAL. OFFICE BUILDINGS

ELECTR ICITY

MEAN STD.DEV.

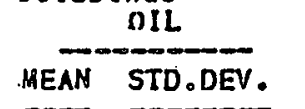

TOTAL

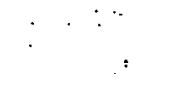

NEW ENGLAND

73.6

0.4

107.121 .1

$180.7 \quad 20.6$

MI DDLE..ATLANTIC

$62: 1$

17.8

$80.0 \cdot 12.5$

151.6

16.2

EAST NORTH CENTRAL

$68.0 \quad 22.8$

$66.2 \quad 39.6$

155.6

7.2 .0

WEST. NORTH CENTRAL

$59.4 \quad 34.5$

33.9

25.9

117.0

39.4

SOUTH ATLANTIC

75.436 .3

$53.9: 56.1$

139.0

62.6

EAST SOUTH CENTRAL

126.0

0.0

48.5

0.0

231.2

0.0

WEST SUUTH CENTRAL

0.0

0.0

0.0

0.0

0.0

0.0

MOUNTAIN

0.0

0.0

0.0

$: 0.0$

0.0

0.0

PACIFIC

39.1

3.5

77.0

22.2

116.1

.25 .7

NATIUNAL AVERAGE

$72.5 \quad 33.6$

58.5

50.4

1.42 .5 .59 .1 
TARBRE II - D2A

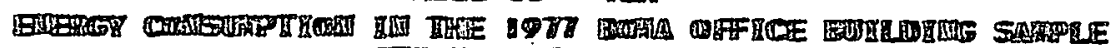

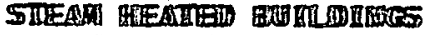

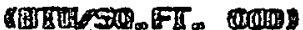

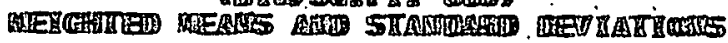

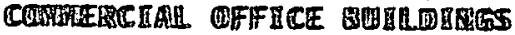

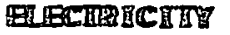

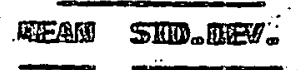

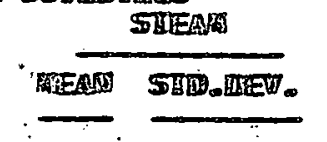

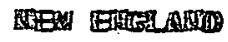

$$
602
$$

56.

1003.7

04.4

7010.4 IVI

THEAN SHDP

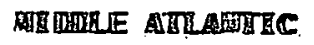

76.2 .2303

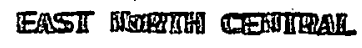

매. 7

28:- 7

78.10

48.5

(1)

35.7

167.9

06.4

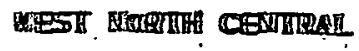

67.9

27.4

7503.26 .6

68.4

82.1

B 4)

3 .

FUUTH: AILARERC

1000.2

1.7 $D$

30.7

19.5

150

0.25

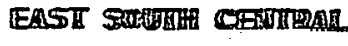

8505

1204

510

8.7

1. 35.3

43.3

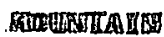

ถาก 5

27.2

537.6

3.64

720

12.

$377-$

$-750 \quad \ldots \quad 26.1$

16. 2

200

40.6

$4.47,3$

$\$ 7 \infty 6$

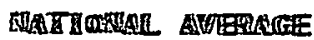

$$
\text { - }
$$

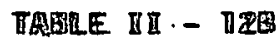

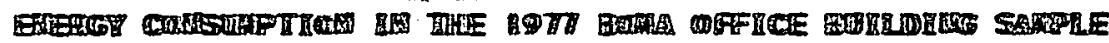

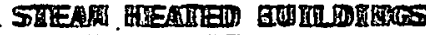

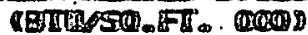

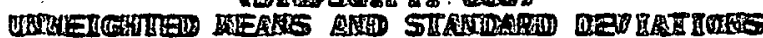

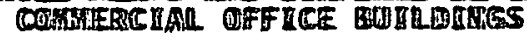
EELETIRICETY STIEAM

TOTRAL

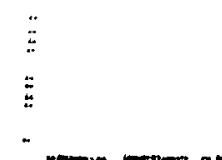

-."

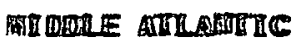
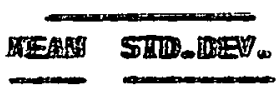

42. 7

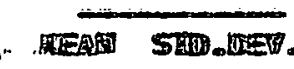

140 2

30.5

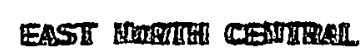

75.4

78.58 .5

48.3

84.2 $-24.7^{\circ}$

93. 7

89.5

T2.6

65

PACREFC

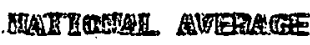

18.3

27.2

320

24.6

180.4

32.0
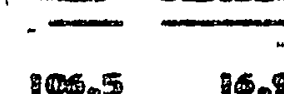

64.65

16.9

300.4

78.5 - " 5

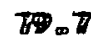

42

$-450$

377.

6อ.

20.(1).

.483

130

56003

42

2000

64

48

300
.
1316.5 5

307

948 0 32. 7

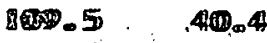

148.4

40 


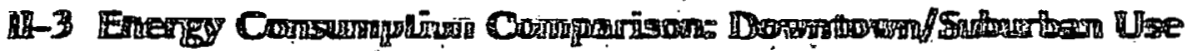

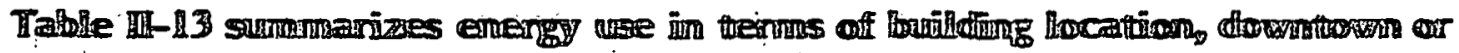

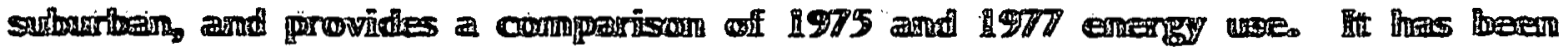

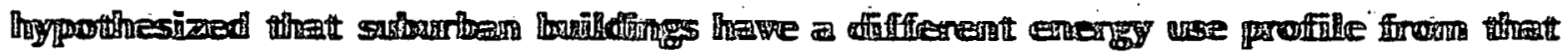

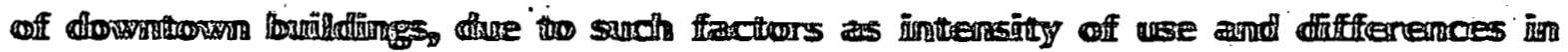

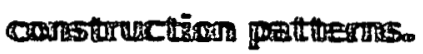

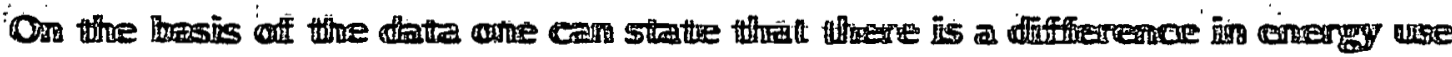

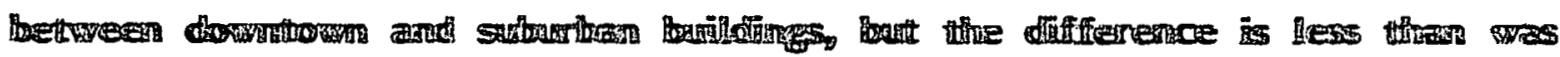

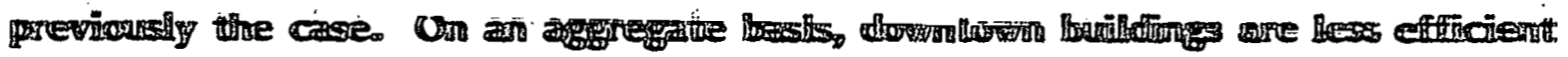

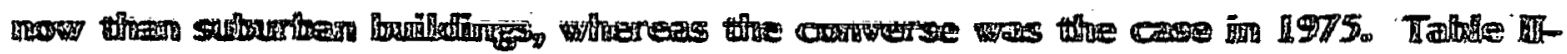

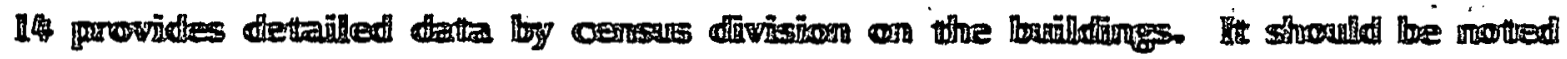

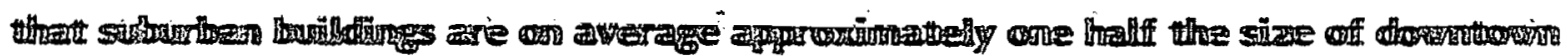

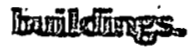

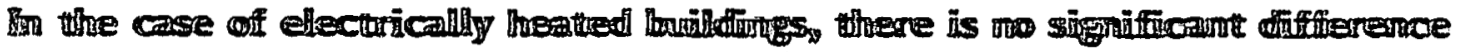

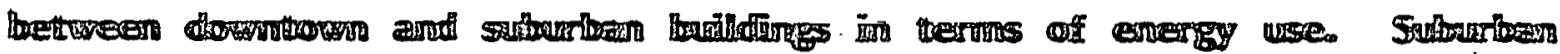

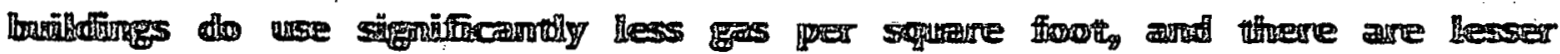
Af

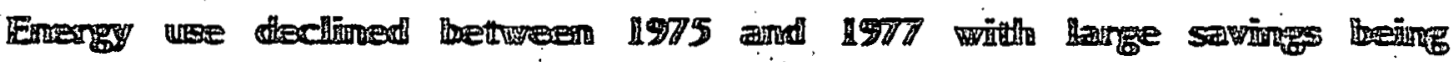

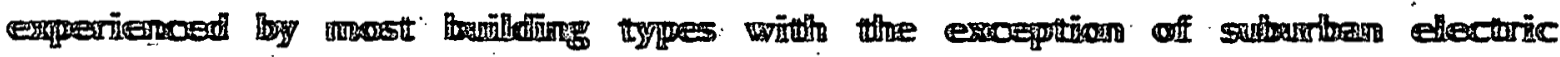

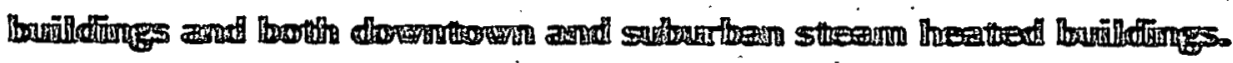


TABLE II-13

COMPARISON OF ENERGY CONSUMPTION:

DOWNTOWN AND SUBURBAN COMMERCIAL OFFICE BUILDINGS

Downtown Buildings

\begin{tabular}{|c|c|c|c|}
\hline \multicolumn{2}{|c|}{ Downtown Buldangs } & \multicolumn{2}{|c|}{ Dur Dan Bullaings } \\
\hline 非 B & $10^{3} \mathrm{Btu} / \mathrm{Sq} . \mathrm{Ft}$. & \#| Bui & $10^{3} \mathrm{Btu} / \mathrm{Sg} . \mathrm{Ft}$. \\
\hline 1975 & $75 \quad 1977$ & $\sqrt{975}$ & 1975 \\
\hline
\end{tabular}

Electric
Gas
Oil
Steam
Average Across
All Fuels

$\begin{array}{rrrrrrrr}24 & 87 & 145.5 & 106.4 & 17 & 85 & 104.7 & 103.3 \\ 93 & 235 & 191.9 & 172.0 & 46 & 124 & 248.8 & 157.3 \\ 26 & 53 & 217.8 & 148.1 & 4 & 18 & 181.5 & 144.0 \\ 158 & 282 & 132.6 & 134.8 & 3 & 5 & 128.9 & 128.3 \\ 301 & 657 & 156.7 & 144.5 & 70 & 232 & 191.6 & 135.5\end{array}$


TABLE II - $14 \mathrm{~A}$.

DOWNTOWN AND SUBURBAN BUILDINGS IN THE IO 77 BOMA
OFFICE BUILDING SAMPLE.

WEIOHTED MEANS AND STANDARD DEVIATIONS

COMMERCIAL OFFICE BUILDINGS

NATIONAL

TYPE OF HEATINO FUEL.

LUCATION OF

BUILDINGS

TYPE OF HEATINO FUEL

DOWNTOWN

TOTAL BTU/SO.FT. (000)

STANDARD DEVIATION 1000

SOUARE FEET $(000,000)$

. NUMBER :OF BUILDINGS

SO.FT.JBLDG. (000)

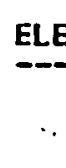

$\begin{array}{rrrrr}106.4 & 172.0 & 148.1 & 134.8 & 144.5 \\ 51.4 & 128.0 & 69.2 & \therefore 56.6 & 89.7 \\ 22.5 & 62.5 & 12.6 & 92.7 & 1.90 .3 \\ 87.0 & 235.0 & 53.0 & .282 .0 & .657 .0\end{array}$

SUBURBAN

TOTAL BTU/SO.FT. $(000)$.

259.0

266.0

238.2

328.6

289.7

STANDARD DEVIATIUN 1000

SOUARE FEET $(000,000)$

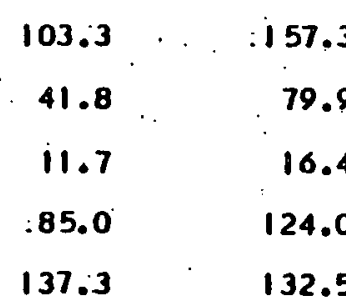

i 44.0

128.3

135.5

NUMBER IF BUILDINAS

SO.FT./BLDG. (000)

137.3

132.5

52.7 .

0.7

68.7

3.4

1.4

. 32.9

18.0

: 5.0

232.0

189.5

.273 .3

141.7 
IABLE \\. 14A, Continued

DOWNTOWN AND SUBURBAN BUILDINOS IN THE 1977 BOMA

OFFICE BUTLDINO SAMPLE

WEIOHTED MEANS AND STANDARD DEVIATIONS

COMMERCIAL OFFICE BUILDINGS

NEW ENCLAND

\section{LUCATIUN UF BUILDINOS}

DOWNTOMN

TOTAL BTU/SO;FT. $(000)$

STANDARD DEVIATION 1000

SOUARE FEET $(000.000)$

NUMBER OF BUILDINGS

SQ.FT. $/ B L D O .(000)$

SUBURBAN

TOTAL BTU/SO.FT. $(000)$

STANDARD DEVIATION 1000

SOUARE FEET $(000,000)$

NUMBER OF BUILDINTSS

SO.FT.ABLDG. $(000)$

TYPE OF HEATINO FUEL

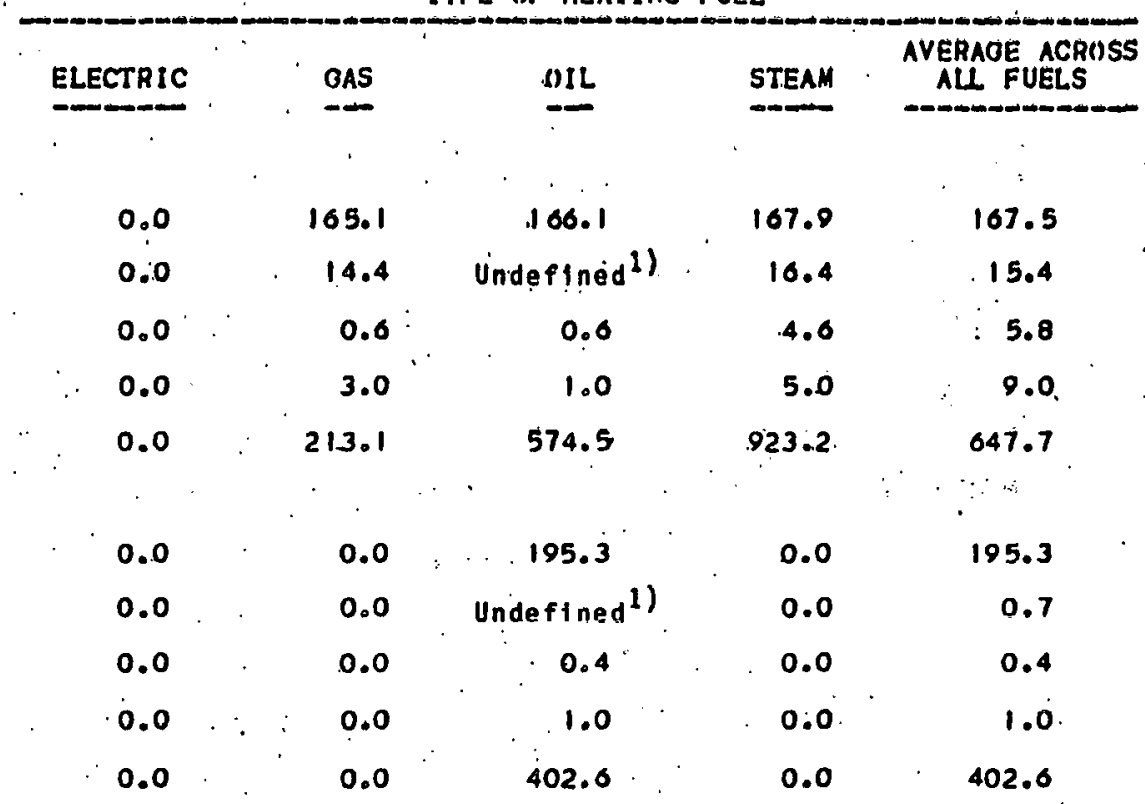

TABLE II - 14A, Continued

DOWNTOWN AND SUBURBAN BUILDINGS IN THE 1977 BOMA

OFFICE BUILDING SAMPLE

HEIGHTED MEANS AND STANDARD DEVIATIUNS

COMMERCIAL OFFICE BUILOINGS

MIDDLE. ATLANTIC

TYPE OF HEATINO FUEL

LOCATION OF

BUILDINGS

DOMNTOWN

TUTAL BTU/SO.FT. (000)

STANDARD DEVIATION 1000

SOUARE FEET $(000,000)$

NUABER OF BUILDINGS

SO.FT./BLDG. (000)

SUBURBAN

TUTAL BTU/SO.FT. (OOO)

STANDARD DEVIATION 1000

SOUARE FEET $(000,000)$

NUMBER OF BUILDINGS

SN.FT./BLDG. $(000)$

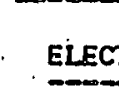

.

$0.0 \quad 179.7$

132.5

136.7

138.1

0.0

64.0

46.4

53.8

54.6

0.0

1.4

0.5

43.4

45.4

0.0

7.0

3.0

80.0

90.0

0.0

206.8

175.6

542.8

504.5

$\begin{array}{cr}181.7 & 196.4 \\ \text { Undefined } 1) & 90.9 \\ 0.0 & 0.9 \\ 1.0 & 9.0 \\ 4.0 & 104.5\end{array}$

.156 .2

128.9

156.9

16.5

5.6

63.3

0.3

1.3

2.6

3.0

4.0

17.0

331.8

.153 .1 
TABLE II - 14A, Contlnued

DCONTIUHN AND SUBURBAN BUILDINCS IN THE 1077 BOMA

OFFICE BUILDINO SAMPLE

WEIOHTED MEANS AND STANDARD DEVIATIONS

COMMERCIAL OFFICE BUTLOIMGS

.EASI NORTH CENTRAL

WOCATION OF
BUILDINGS

DINNTOHN

TOTAL BTU/SO.FT. $(000)$,

STANDARD DEVIATION 1000

SOUARE FEET $(000,000)$

NUMBER. OF BUILDINGS

SQ.FT.JBLDG. $(000)$

SUBURBAN

TUTAL BTU/SO.FT. (000)

STANDARD DEVIATION 1000

SOUARE FEET $(000,000)$

NUHBER .IF BUILDINGS

SO.FT.ABDO. $(000)$

TYPE OF HEATINO FUEL

\begin{tabular}{|c|c|c|c|c|c|c|}
\hline ELECTRIC & & GAS & . & :OIL & STEAM & $\begin{array}{l}\text { AVERAOE ACROSS } \\
\text { ALL FUELS }\end{array}$ \\
\hline & . & & $\therefore$ & & . & \\
\hline 103.5 & & .157 .2 & $:$ & 168.3 & 150.1 & 148.1 \\
\hline 46.8 & $\therefore$ & 78.9 & $\because$ & 74.6 & 63.4 & 7.2 .0 \\
\hline 4.4 & & 13.2 & & 2.7 & 10.8 & $31: 1$ \\
\hline .9 .0 & & 38.0 & . & 6.0 & .52 .0 & 105.0 \\
\hline 488.7 & . & 346.4 & & .452 .1 & .207 .4 & 295.8 \\
\hline & .. & & . & & & . \\
\hline 106.0 & & 189.6 & & 0.0 & 0.0 & 170.1 \\
\hline 41.8 & & 68.8 & & 0.0 & 0.0 & 72.7 \\
\hline 0.9 & & 2.9 & & 0.0 & 0.0 & 3.7 \\
\hline 7.0 & . & 23.0 & & 0.0 & 0.0 & 30.0 \\
\hline 124.3 & & 124.4 & & 0.0 & 0.0 & 124.4 \\
\hline
\end{tabular}

TABLE II - 14A, Continued

DOHNTINN AND SUBURBAN BUILDINCS IN THE 1977 BOMA

OFFICE BUILDING SAAPLE

HEIGHTED HEANS AND STANDARD DEVIATIONS

COMMERCIAL OFFICE BUILDINGS

WEST MORTH CENTRAL

LOCATION OF
BUILDINGS

DOWNTUWN

TOTAL BTU/SO.FT. (O00)

STANDARD DEVIATIUN 1000

SDUARE FEET $(000,000)$

NUMBER OF BUILDINGS

SO.FT./BLDG. (000)

SUBURBAN

TOTAL BTU/SO.FT. $(\infty 00)$

STANDARD DEVIATION 1000

SDUARE FEET $(000,000)$

NUABER UF BUILDINOS

SO.FT. ABLDG. $(000)$

\section{ELECTRIC}

-

134.8

25.1

1.1

4.0

281.2

1.24 .1

.29 .9

1.1

7.0

163.7

\section{TYPE OF HEATING FUEL}

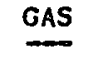

OIL

STEAM

AVERAGE ACROSS

ALL. FUELS

49.7

135.3

.158 .9

48.0

43.3

94.2

0.2

9.6

16.8

2.0

42.0

75.0

78.8

229.4

223.6

216.7

152. 1

0.0

109.2

24.8

0.0

75.1

0.5

0.0

2.9

2.0

0.0

25.0

235.8

0.0

110.5 
TABLE II - 14A, Continued

DOMNTOWN AND SUBURBAN BUILDINGS IN THE 1977 BOMA

OFFICE QUILDING SAMPLE

WEIGHTED MEANS AND STANOARD DEVIATIONS

COMMERCIAL OFFICE BUILOINGS

SOUTH ATLANTIC

LOCATION. OF

BUILDINGS :

DOWNTOWN

TOTAL BTU/SO.FT. $(000)$

STANDARD DEVIATION 1000

SOUARE FEET $(000,000)$

NUMBER OF BUILDINOS

SO.FT./BLDG. $(000)$

SUBURBAN

TOTAL BTU/SO.FT. $(000)$

STANDARD DEVIATION 1000

SOUARE FEET $(000,000)$

NUABER .OF BUILDINGS

SQ.FT./BLDG. $(000)$

TYPE OF HEATING FUEL

\begin{tabular}{|c|c|c|c|c|}
\hline ELECTRIC & GAS & $01 \mathrm{IL}$ & STEAM. & $\begin{array}{l}\text { AVERAGE ACROSS } \\
\text { ALL FUELS }\end{array}$ \\
\hline 96.5 & 162.3 & 140.5 & 142.4 & 130.0 \\
\hline$: 45.7$ & 6.3 .3 & -61.8 & 100.0 & 72.1 \\
\hline 7.0 & 3.0 & 7.6 & 4.8 & 22.4 \\
\hline 31.0. & 19.0 & 38.0 & 13.0 & 101.0 \\
\hline 226.7 & 156.5 & 199.5 & 370.1 & 221.7 \\
\hline 1.12 .0 & 121.4 & 131.1 & 106.4 & 118.7 \\
\hline 31.7 & 72.1 & 58.8 & Undetined 1 ) & .48 .1 \\
\hline 4.4 & 0.9 & 2.2 & 0.0 & 7.5 \\
\hline .37 .0 & 19.0 & 11.0 & 1.0 & 68.0 \\
\hline 117.9 & 49.9 & 199.4 & 39.4 & 110.9 \\
\hline
\end{tabular}

TABLE II - 14A, Continued DOMNTONN AND SUBURBAN BUILDINGS IN THE 1977 BOMA OFFICE BUILDING SAMPLE 'WETOHTED MEANS AND STANDARD DEVIATIONS COMMERCIAL OFFICE BUILOINGS

EAST SUUTH CENTRAL

TYPE OF HEATING FUEL

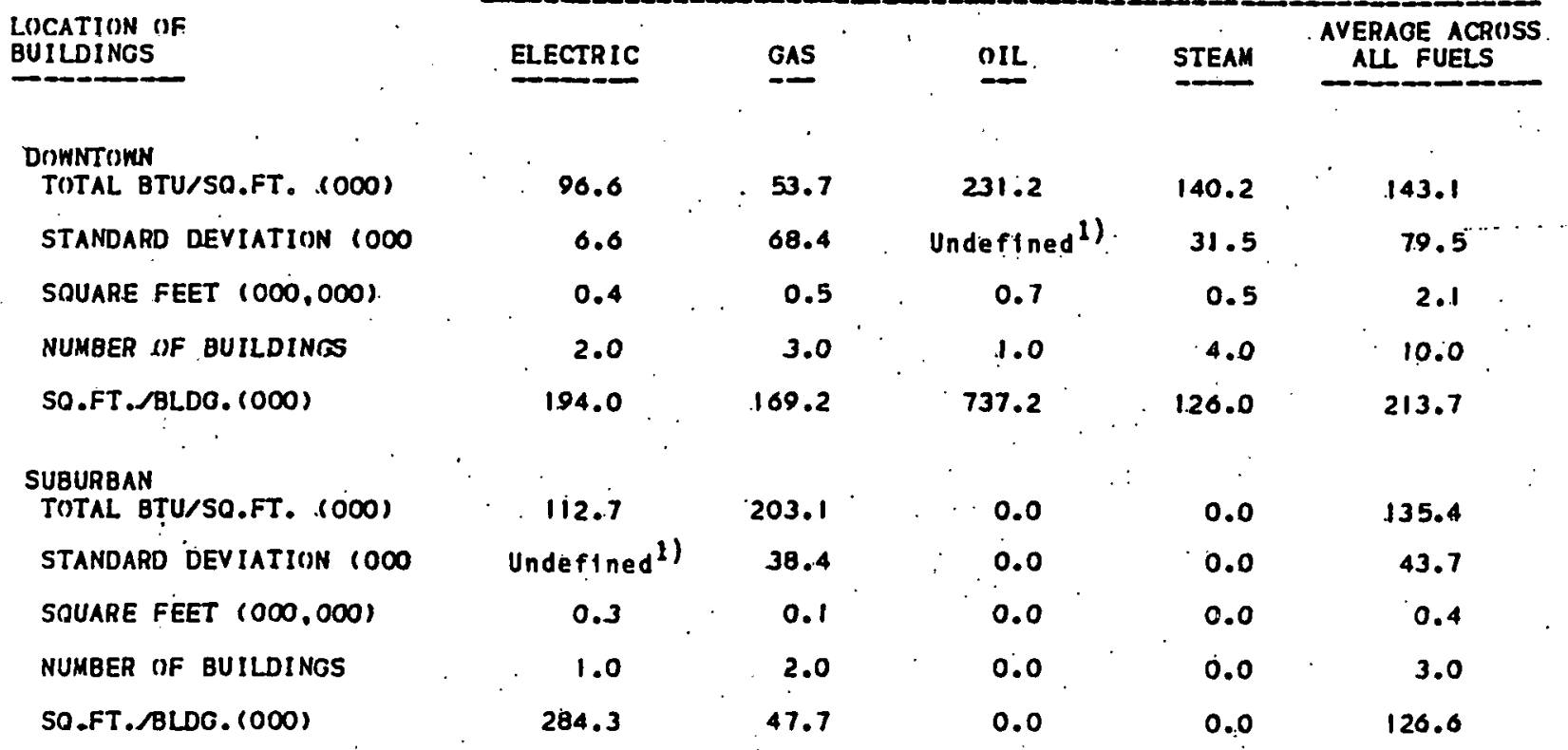

1) One butlding. 
TABLE II - 14A, Continued

DOWNTUMN AND SUBURBAN BUILDINGS IN THE 1977 BOMA

OFFICE BUILDING SAMPLE

WEIGHTED MEANS AND STANDARD DEVIATIONS

COMMERCIAL OFFICE BUILDINGS

WEST SUUTH CENTRAL.

TYPE OF HEATING FUEL

LOCATION IIF:

BUILDINGS

DOWNTOWN

TUTAL BTU/SQ.FT. $(000)$

STANDARD DEVIATION 1000

SOUARE FEET $(000,000)$

NUMBER OF BUILDINGS.

SO.FT. . RLDG..(000)

SUBURBAN

TUTAL BIU/SO.FT. $(000)$

STANDARD DEVIATION 1000

SOUARE FEET $(000,000)$

NUMBER IF BUILDIÑGS

SO.FT.ABLDG. (000)

\section{EL}

$\begin{array}{rrrrrr}140.5 & 200.4 & & 0.0 & 136.5 & 188.1 \\ 40.7 & 165.5 & 0.0 & 3.7 & .154 .2 \\ 1.9 & \because 19.3 & 0.0 & 1.8 & 23.2 \\ 7.0 & 055.0 & 0.0 & 2.0 & .65 .0 \\ 276.4 & 350.2 & 0.0 & 875.5 & 357.4\end{array}$

$\begin{array}{ccccc}86.5 & 153.7 & 0.0 & 0.0 & 131.3 \\ 48.8 & 82.0 & 0.0 & 0.0 & 79.3 \\ 3.0 & 5.9 & 0.0 & 0.0 & 8.9 \\ 15.0 & 29.0 & 0.0 & 0.0 & 44.0 \\ 198.0 & 204.5 & 0.0 & 0.0 & 202.2\end{array}$

TABLE II - I4A, Continued

DOWNTOWN AND SUBURBAN BUILDINGS IN THE 1977 BOMA OFFICE BUILDING SAMPLE WEIGHTED MEANS AND STANDARD DEVIATIONS

COMMERCIAL OFFICE BUILDINGS M(I)UNTAIN

\section{LOCATION OF BUILDINGS}

\section{DUMNTUMN}

IUTAL BTU/SO.FT。(000)

STANDARD DEVIATION $\mathrm{COOO}$

SOUARE FEET $(000,000)$

NUMBER OF BUILDINGS

SO.FT. .BLDG. $(000)$

SUBURBAN

TUTAL BTU/SO.FT. .(OOO)

STANDARD DEVIATION COOO

SOUARE FEET $(000,000)$

NUMBER OF BUILDINGS

SO.FT. ABLDG. (000)
TYPE OF HEATING FUEL

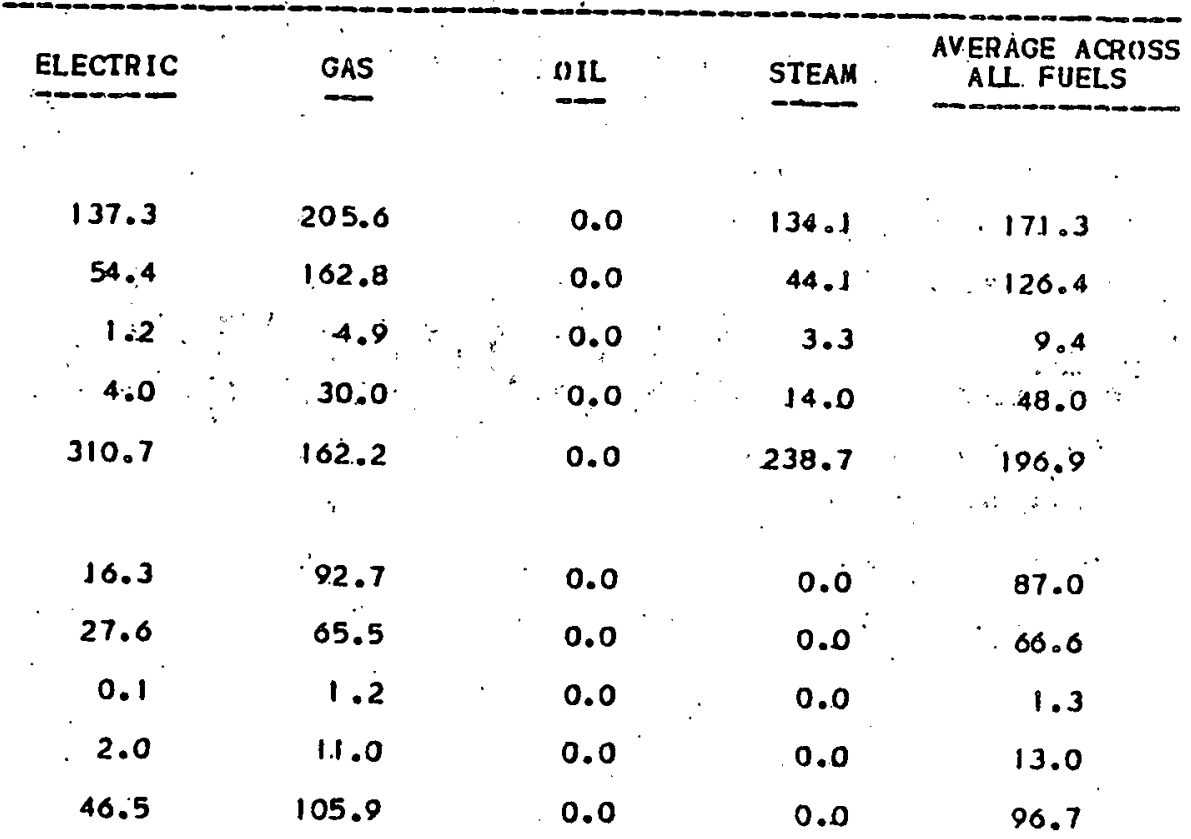


TABLE II = 1AA, Continuge

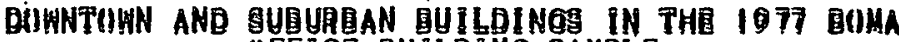

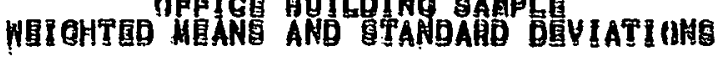

COAMEAGIAL OPFICE BUILBIHAS

PAEIPIC

TYPG M HATINO FUEL

\section{LUEATION () \\ UILEINOS}

\section{D()HNTOHN}

TITAL OTU/RO.FT. 1000 )

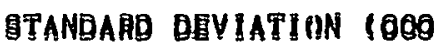

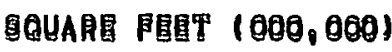

NUMAER MP OUILOINGS

G0.

SUIOARAN

TOTAL OTU/80, BT: $(060)$

OTANDARQ QRVIATIIN 1000

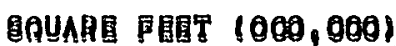

NUM国 of OUILOINOS

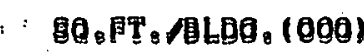

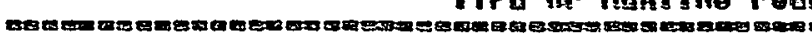

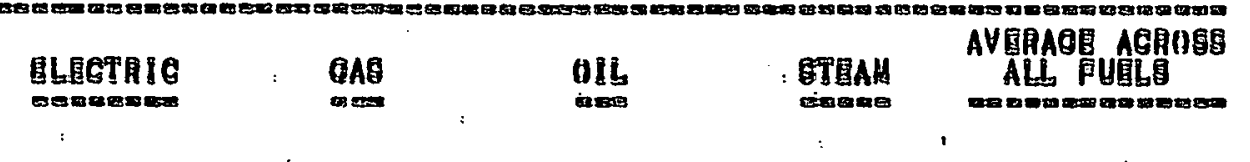

\begin{tabular}{|c|c|c|c|c|}
\hline 98.6 & 12683 & $9 \theta_{e} 0$ & 109.4 & 111.9 \\
\hline 98.7 & 80. 1 & Undef:|ned & 47.0 & 60.1 \\
\hline 6.4 & 13.6 & 0,0 & 19,8 & 34.1 \\
\hline 39.0 & 93.6 & $1: 0$ & 70.6 & $=15.0$ \\
\hline & $260_{0} 4$ & $4 \dot{7}, 5$ & 197.1 & 221.8 \\
\hline
\end{tabular}

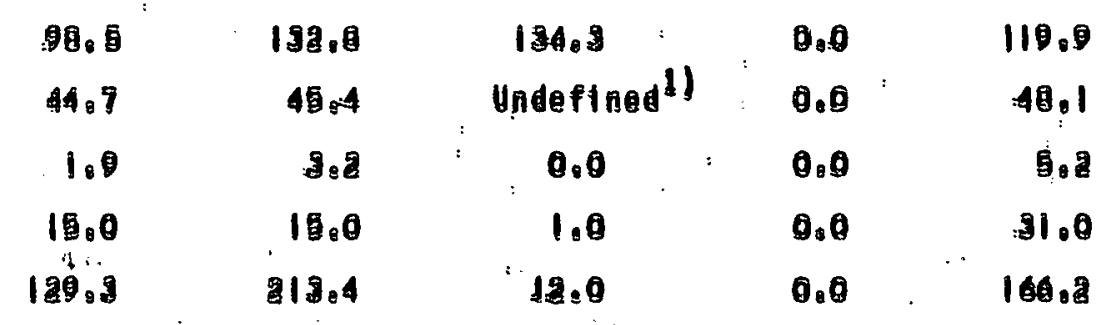

\footnotetext{
1) On日 gutiding.
} 
TABLE II: : 148

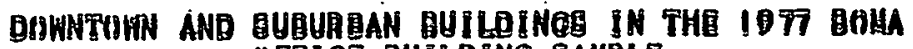

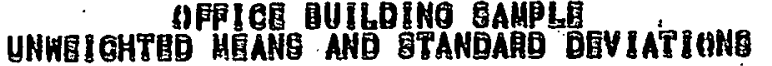

COHAERCIAL OPFIGE OULLOHAB

NATIUNAL

TYPG OP: HATINO FU'⿺

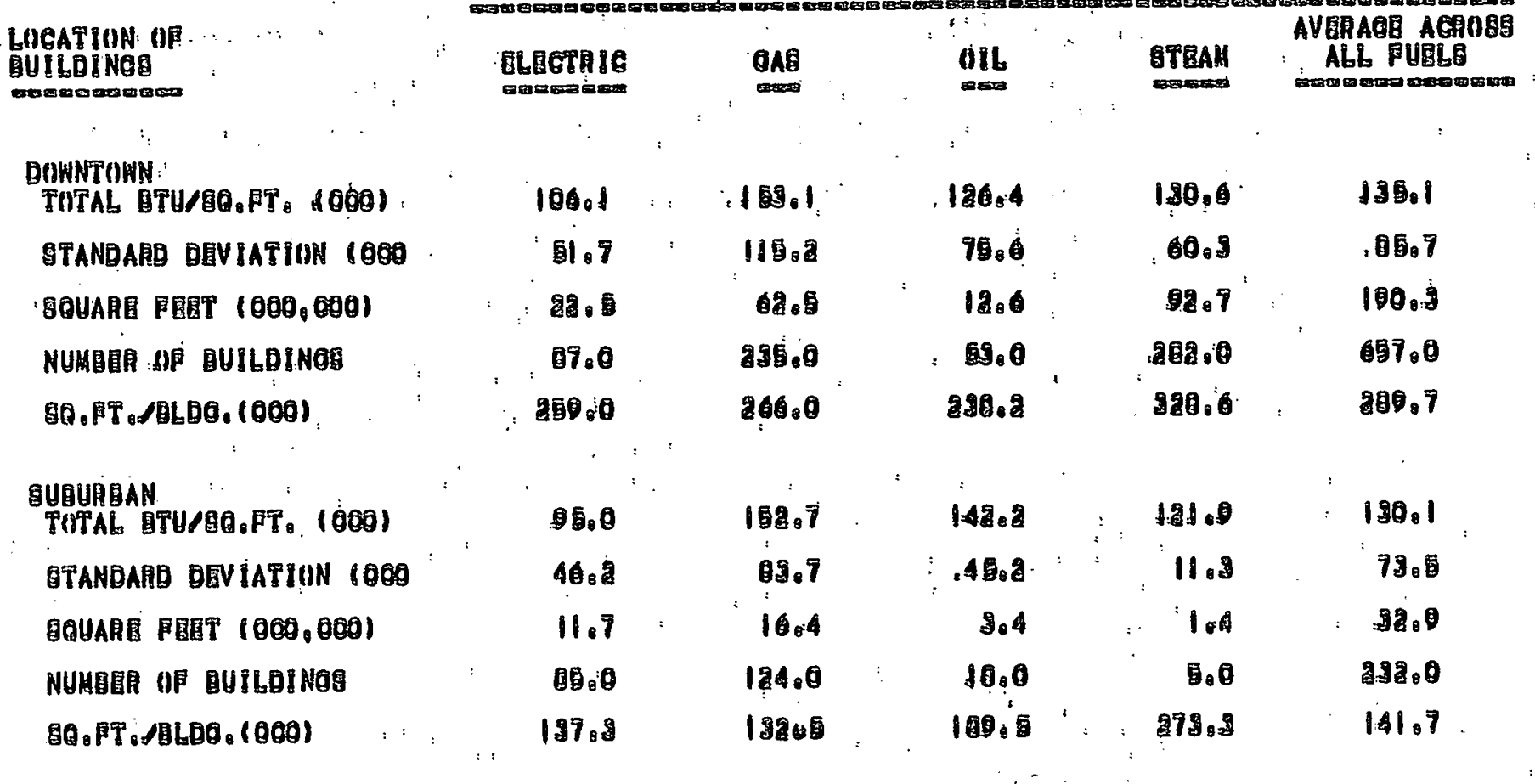


TABLE II - 14B, Continued

DOWNTOWN AND SUBURBAN BUILDINGS IN THE 1.977 BOMA

OFFICE BUILDING SAMPLE

UNWEIGHTED MEANS AND STANDARD. DEVIATIONS

COMMERCIAL OFFICE BUILDINGS

NEN ENGLAND

TYPE SF HEATING FUEL

LOCATION OF
BUILDINGS

\section{ELECTRIC}

GAS

165.3

17.9

0.0

0.0

0.6

0.0

3.0

0.0

213.1

0.0

0.0

0.0

0.0

0.0

$\begin{array}{ll}0.0 & 0.0 \\ 0.0 & 0.0 \\ 0.0 & 0.0 \\ 0.0 & 0.0 \\ 0.0 & 0.0\end{array}$

195.3

0.0

0.4

1.0

402.6

\section{STEAM}

AVERAGE ACROSS

AU FUELS

SO.FT . $/ B L D G .(000)$

\section{LOCATION. OF \\ BUILDINGS}

DOWNT:UNN

TUTAL BTU/SO.FT. $(000)$

STANDARD DEVIATION COOO

SQUARE FEET $(000 ; 000)$

NUMBER $\leadsto F$ BUILDINOS

SO. FT a $/ B L D G$. $(000)$

SUBURBAN

TUTAL BTU/SO.FT. (000)

STANDARD DEVIATIIIN 1000

SOUARE FEET $(000,000)$

NUXBER OF BUILDINGS

SO.FT./BLDG。(000)
TABLE II - 14B, Continued

DONNTUWN AND SUBURBAN BUILDINGS IN THE 1977 BIMA

OFFICE BUILDING SAMPLE UNWEIGHTED MEANS AND STANDARD DEV IATIONS

COMMERCIAL OFFICE BUILDINGS

MIDOLE ATLANTIC

TYPE OF HEATINO FUEL

ERETRIC

GAS

IIL

STEAM

0.0

0.0

195.3

0.0

0.0

0.4

0.0

1.0

0.0

.402 .6

GECTRIC . IIL STEAM AVERAGE ACRUSS

$\begin{array}{rrrrrr}0.0 & 132.7 & 102.0 & 128.5 & 128.0 \\ 0.0 & 102.9 & 89.0 & 53.3 & 58.7 \\ 0.0 & 1.4 & 0.5 & 43.4 & .45 .4 \\ 0.0 & 7.0 & 3.0 & 80.0 & 90.0 \\ 0.0 & 206.8 & 17.5 .6 & 542.8 & 504.5\end{array}$

$\begin{array}{rr}181.7 & 160.5 \\ 0.0 & 67.1 \\ 0.0 & 0.9 \\ 1.0 & 9.0\end{array}$

150.0

$125.8 \ldots: 151.7$

$4.0 \quad .104 .5$

19.5

8.4

50.8

0.3

1.3

.2 .6

3.0

4.0

17.0

110.6

331.8

153.1 
TABLE II - 14B, Continued

DOWNTOWN AND SUBURBAN BUILDINGS IN THE 1977 BOMA

OFFICE BUILDING SAMPLE

UNWEIGHTED MEANS AND STANDARD DEVIATIONS

COMMERCIAL OFFICE BUILDINGS

EAST NOHTH CENTRAL

LOCATION OF
BUILDINGS

DOWNTOWN

TUTAL BTU/SO.FT. (000)

STANDARD DEVIATION 1000

SOUARE FEET $(000,000)$

NUMBER UF BUILDINGS

SO.FT./BLDG. $(000)$

SUBURBAN

TUTAL BTU/SO.FT. (000)

STANDARD DEVIATION 1000

SOUARE FEET $(000,000)$

NUMBER OF BUILDINGS

SO.FT._BLDG. (000)
TYPE UF HEATING FUEL
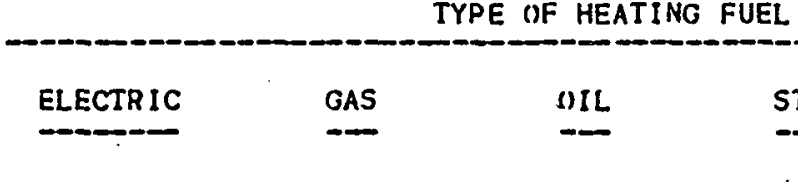

$-\infty$

SIIL
$: 157.6$

78.0

13.2

38.0

488.7

346.4

.103 .0

167.3

23.0

124.3

124.4
0.0

0.0

0.0

0.0

155.6
72.0
2.7
6.0
452.1
0.0
0.0
0.0
0.0
0.0

0.0
STEAM

1.46 .8

7.4 .6

10.8

52.0

207.4

0.0

152.3

0.0

0.0

68.1

3.7

0.0

30.0

0.0

124.4

TABLE II - 148, Continued

DOWNTOWN AND SUBURBAN BUILDINGS IN THE 1977 BOMA OFFICE BUILDING SAMPLE UNWEIGTED MEANS AND STANDARD DEVIATIONS COMMERCIAL OFFICE BUILDINGS

WEST NORTH CENTRAL

TYPE OF HEATING FUEL

\section{LOCATION OF BUILDINGS}

DOMNTOWN

TOTAL BTU/SO.FT. $(000)$

STANDARD DEVIATION 1000

SOUARE FEET $(000,000)$

NUMBER OF BUILDINGS

SO.FT./BLDG . (000)

SUBURBAN

TOTAL BTU/SO.FT. $(000)$

STANDARD DEVIATION 1000

SOUARE FEET $(000,000)$

NUMBER OF BUILDINGS

SO.FT./BLDG.(000)

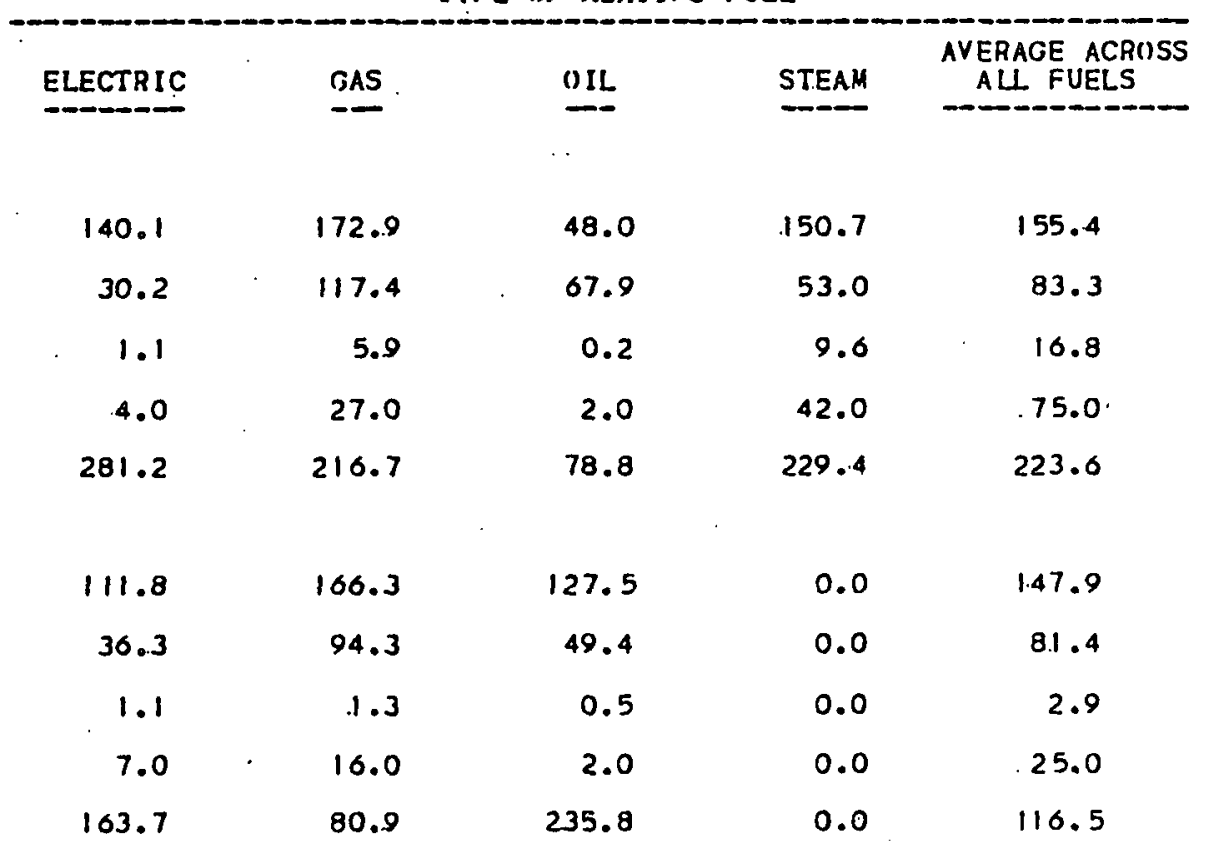


TABLE II - 14B, Continued

DUWNTOWN AND SUBURBAN BUILDINOS IN THE 1.977 BISMA

OFFICE BUILDINO SAMPLE

UNHEIGHTED MEANS AND STANDARO DEVIATIONS

COMMERCIAL OFFICE BUILDINGS

SOUTH ATLANTIC

\begin{abstract}
LUCATIUN OF BUILDINGS
\end{abstract}

DONNTOWN

TOTAL BTU/SO.FI. 10008

STANDARD DEVIATION 1000

SOUARE FEET $(000,000)$

NUMBER OF BUILDINGS

$50 . F T . A B L D,(000)$

SUBURBAN

TUTAL BTU/SO.FT. $(000)$

STANDARD DEVIATION 1000

SOUARE FEET $(000,000)$

NUMBER OF BUILDINGS

3Q.FT./BLDG . (000)

TYPE OF HEATINO FUEL

\begin{tabular}{llll} 
ELECTRIC OVIL & AVERAGE ACROSS \\
\hline
\end{tabular}

$\begin{array}{rrrrr}93.6 & 136.2 & 128.1 & .156 .7 & 122.7 \\ 47.2 & 63.8 & .73 .8 & 89.9 & 69.6 \\ 7.0 & 3.0 & 7.6 & 4.8 & 22.4 \\ 3.1 .0 & 19.0 & 38.0 & 13.0 & 101.0 \\ 226.7 & 156.5 & 199.5 & 370.1 & 221.7\end{array}$

$\begin{array}{rrrrr}96.9 & 1.39 .6 & 138.6 & 106.4 & 115.7 \\ 40.2 & 89.3 & .52 \% 9 & 0.0 & 62.3 \\ 4.4 & 0.9 & 2.2 & 0.0 & 7.5 \\ 37.0 & 19.0 & 11.0 & 1.0 & 68.0 \\ 117.9 & 49.9 & 199.4 & 39.4 & 110.9\end{array}$

TABLE II - 14B, Continued DOWNTOWN AND SUBURBAN BUILDINGS IN THE 1977 BOMA OFFICE BUILDING SAMPLE UNWEIGHTED MEANS AND STANDARD DEVIATIONS COMMERCIAL OFFICE BUILDINGS

EAST SUUTH CENTRAL

TYPE OF HEATING FUEL

LOCATION OF BUILDINGS

DONNTOWN

TOTAL BTU/SQ.FT. $(000)$

STANDARD. DEVIATION 1000

SQUARE FEET $(000,000)$

NUMBER OF BUILDINGS

SO.FT./BLDG. (000)

SUBURBAN

TUTAL BTU/SO_FT. (000)

STANDARD DEVIATION .000

SOUARE FEET $(000,000)$

NUMBER . OF BUILDINGS

SO.FT . /BLDG. $(000)$
GAS

(IL

ELECTRIC

99.6

96.0

10.3

0.4

89.6

2.0

0.5

3.0

169.2

1.12 .7

0.0

$: 156.5$

0.0

85.4

0.0

0.1

0.0

2.0

1.0

47.7

$$
\begin{array}{r}
231.2 \\
0.0 \\
0.7 \\
1.0
\end{array}
$$

737.2

0.0

0.0

284.3
STEAM

AVERAGE ACROSS ALL FUELS
125.5

63.2

2.1

10.0

213.7

126.0

141.9

$0.0 \quad .65 .5$

0.0

0.4

3.0

120.6 
TABLE II - 14B, continued

DOIWNTOHN AND SUBURBAN BUILDINOS IN THE 1977 BOMAA

OFFICE BUILDINO SAMPLE

UNWEI GHTED MEANS AND STANDARD DEVIATIONS

COMMERCIAL OFFICE BUILDINGS

WEST SOUTH CENTAAL.

TYPE OFF HEATINO FUEL

LOCATION OF
BUILDINOS

\section{--_-}

DONATEWN

TUTAL BTU/SO.FT. $(000)$

STANDARD DEVIATION 1000

SQUARE FEET $(000,000)$

NUMBER OF BUILDINCS

SO.FT..ALDG. (000)

SUBURBAN

TUTAL BTU/SO.FT. (ODO)

STANDARD DEVIATION 1000

SOUARE FEET $(000,000)$

NU.MBER OF BUILDINGS

SO.FT.ALDG. . (000)

ELECTRIC GAS III

AVERAGE ACROOSS ALL FUELS

TABLE II - 14B, Continued

DOWNTONN AND SUBURBÄN BUILDINGS IN THE 1977 BOMA OFFICE BUILDING SAMPLE UNWEIGHTED MEANS AND STANDARD DEV IATIONS

COMMERCIAL OFFICE BUILOINGS MUUNTAIN

\section{LOCATION OF BUILDINGS}

DOWNTOWN

TOTAL BTU/SO.FT. (000)

STANDARD DEVIATIUN 1000

SOUARE FEET $(000.000)$

NUABER OF BUILDINGS

SO.FT.JBLDG. (000)

SUBURBAN

TUTAL BTU/SQ.FंT. $(.000)$

STANDARD DEVIATION 1000

SQUARE FEET $(000,000)$

NUMBER SF BUILDINGS

SQ.FT. ABLDO. (000)

$\begin{array}{rr}131.7 & 181.1 \\ 60.6 & 147.4 \\ 1.9 & 1.9 .3 \\ 7.0 & 55.0 \\ 276.4 & 350.2 \\ . & \\ 90.6 & .158 .9 \\ 59.7 & 111.0 \\ 3.0 & .5 .9 \\ 15.0 & 29.0 \\ 198.0 & 204.5\end{array}$

0.0 .

137.6

171.6

0.0

5.5

139.3

0.0

1.8

23.2

2.0

.65 .0

0.0

875.5

357.4

0.0

0.0

135.6

0.0

0.0

101.3

0.0

0.0

8.9

0.0

0.0

44.0

0.0

0.0

202.2

TYPE OF. HEATINO FUEL

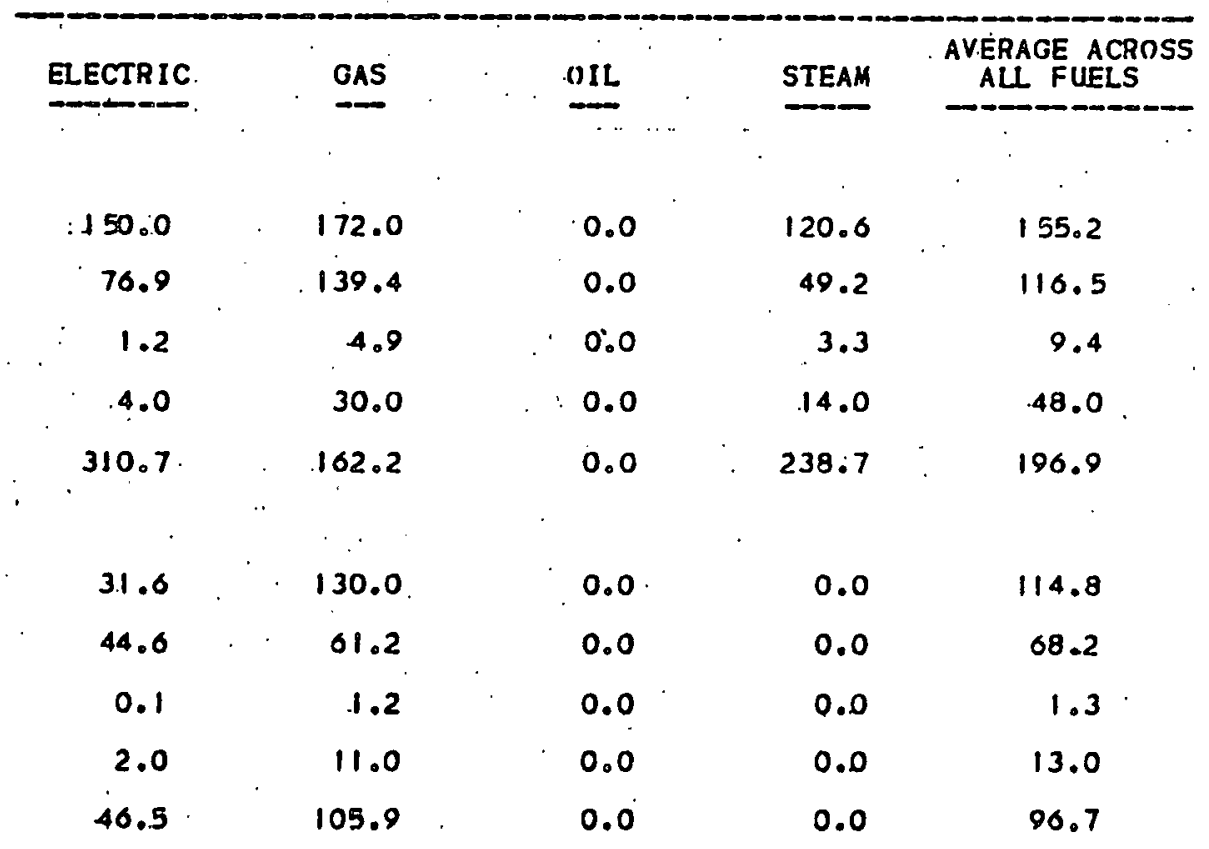


TABLE 11. - 14B, Continued

DOWNTOWN AND SUBURBAN BUILDINGS IN THE 1977 BOHA

OFFICE BUILDING SAMPLE UNWEI GHTED MEANS AND STANDARD DEVIATIONS

COMMERCIAL OFFICE BUILDINGS

PACIFIC

TYPE OF HEATINO FUEL

\section{LOCATION OF \\ BUILDINOS}

\section{DOWNTOWN}

TOTAL BTU/SO.FT. (OOO)

STANDARD DEVIATION 1000

SOUARE FEET $(000,000)$

NUMBER OF-BUILDINGS

SO.FT./BLDG..(000)

SUBURBAN

TOTAL BTU/SO.FT. $(000)$

STANDARD DEVIATION 1000

SOUARE FEET $(000,000)$

NUEBER OF BUILDINOS

SQ.FT.ABLDG. $(000)$

\begin{tabular}{rr} 
ELECTRIC & OAS \\
\hline 106.9 & 111.6 \\
46.4 & 94.0 \\
6.4 & 13.8 \\
30.0 & 53.0 \\
214.0 & 260.4 \\
84.0 & 131.9 \\
43.1 & 51.0 \\
1.9 & 3.2 \\
15.0 & 15.0 \\
129.3 & 213.4
\end{tabular}

OIL

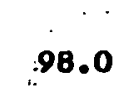

0.0

0.0

1.0

47.5

134.3

0.0

0.0 .

i.o

12.0
STEAM

AVERAGE ACRIISS

AU FUELS

$\begin{array}{rr}102.7 & 106.6 \\ 48.4 & 67.0 \\ 1.3 .8 & 34.1 \\ 70.0 & .154 .0 \\ 197.1 & 221.2\end{array}$

$0.0 \quad 109.1$

$0.0 \quad 51.6$

$0.0 \quad 5.2$

$0.0 \quad 31.0$

$0.0^{\circ} \quad 160.2$ 


\section{II-4 Energy Consumption by Height}

Tables II-15 and II-16 present information on electric, fuel, and total energy use by building height. In general, buildings in excess of 9 stories use more electricity per square foot than do shorter buildings. This may be due to the need for proportionally more elevators and air handling equipment than is the case for shorter buildings. In addition, the taller buildings are located in major cities and may have high energy use activities located within them or may be subject to greater utilization.

There is no clear energy use trend in the case of fuel use, but buildings in the 30-49 story height range tend to use more fuel than do other buildings. Whether these energy patterns are related to the buildings' structural characteristics, to thë buildings' usage, or to the choice of heating fuel has not been determined. The relatively low energy consumption figure for very tall buildings is probably accounted for by the existence of steam heated buildings. 
TABLE II-15

BUILDING ENERGY CONSUMPTION AND HEIGHT OF BUILDING

COMMERCIAL OPTICE BUILDINGS

WEIGHTIZD MEANS

Energy Coneumptlon

$\left(10^{3}\right.$ Btu)

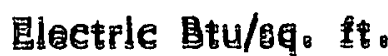

1977
1975

Other Fuel Btu/sg. ft. 1977

1975

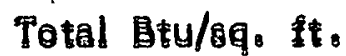

1977

1975

"Number of Bldggs.

1977

1995

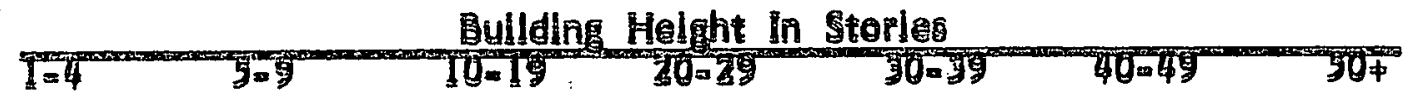

79.

79.2

849

84.7
87.0

92.8
82.2

89.7

79.0

82.7

82.8

93.3

79.

56.4

70.9

$28^{2}$

72.4

82.1

103.6

102.0

28

95.0

50.1

144

$\begin{array}{ll}144.0 & 126.5 \\ 163.3 & 165.1\end{array}$

145.1

158.1

164.4

$180_{0} 0$

181.0

173.8

177.8

i38.

126.1

$\begin{array}{rrrrrrr}120 & 200 & 319 & 115 & 50 & 26 & 18 \\ 34 & 66 & 193 & 72 & 27 & 19 & 20\end{array}$


TABRLE III-IGA

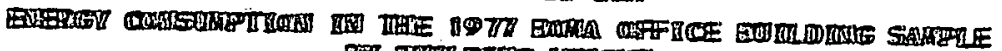

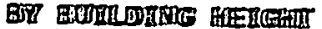

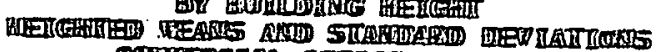

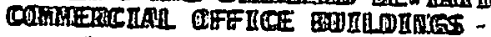

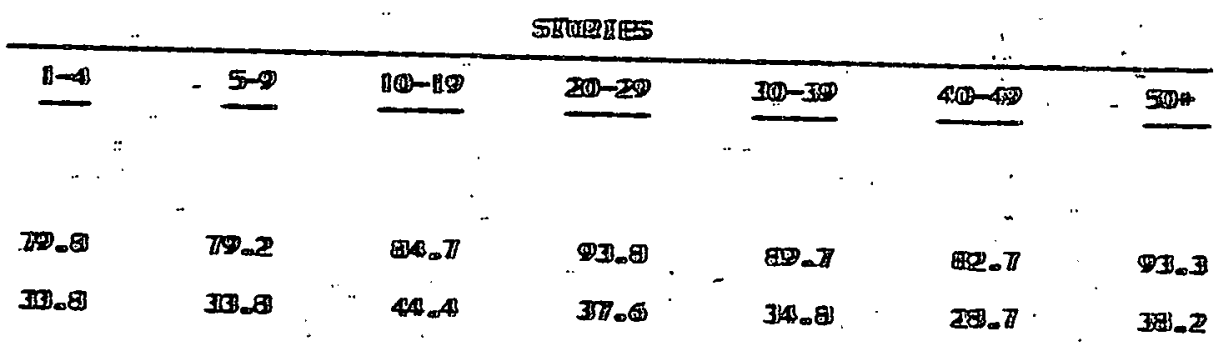

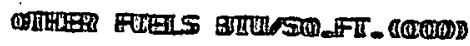

MEAT

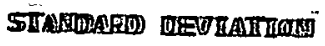

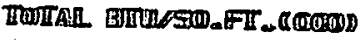

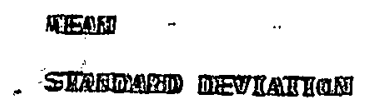

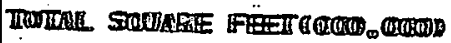

\begin{tabular}{|c|c|c|c|c|c|}
\hline 6 & - 560.4 & 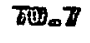 & 72.4 & $n @ x_{-} 6$ & SOS \\
\hline & $4 \times(10)$ & 53 & 800.6 & 17. & 755 \\
\hline
\end{tabular}

CLURB

\begin{tabular}{|c|c|c|c|c|c|c|c|}
\hline 140.0 & & 12465 & .245011 & - & 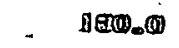 & 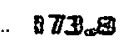 & 0308 \\
\hline $776 \pi_{-\infty} 3$ & & 50.0 & $\therefore \quad-\quad-5$ & बबहा, & 97.5 & - & 42.0 \\
\hline$\$ .5$ & .- & $277_{-}^{\circ}$ & - $590_{\infty}$ & 400. & $30_{\infty} \not 77^{\circ}$ & 228.3 & 24.4 \\
\hline$\Delta 2000$ & & ZாOவ & 31330 & anso" & 5010 & 200 & 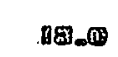 \\
\hline
\end{tabular}

TABBQLE III-IETB

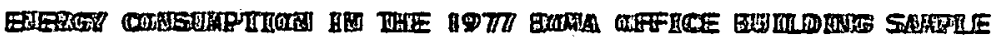
UH

\begin{tabular}{|c|c|c|c|c|c|c|c|}
\hline$B=0$ & -58 & - & $n(1)-129)$ & 2010 & $30-30$ & $400-459$ & - Sidr \\
\hline
\end{tabular}

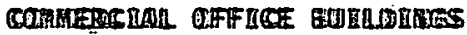

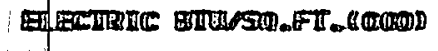

$\therefore$ REAUI

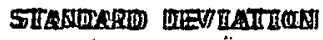

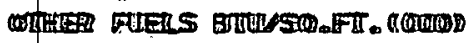

HENOT

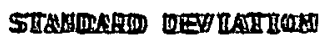

.

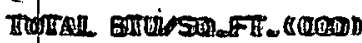

AEENA

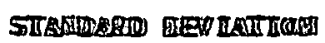

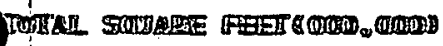

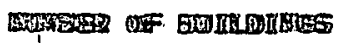

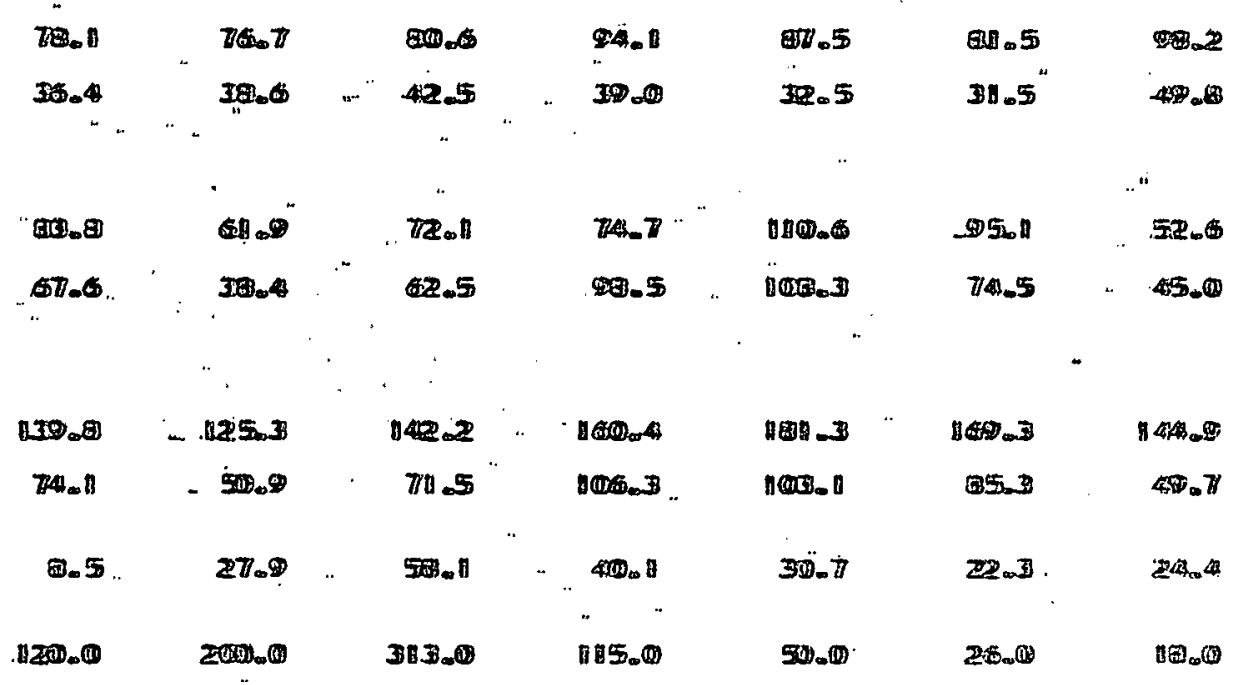




\section{Il-5 Energy Consumption by Building Age}

Table II-17 presents data for building energy use by age for the downtown buildings in the 1977 data base and provides data for comparison with 1975. ${ }^{1}$ ) Additional information for 1977 is available in Table II-18. Energy use is relatively low in new buildings. Low energy use probably is the result of the buildings having heating, ventilating, and air conditioning equipment which is in good operating condition, for it is unlikely that the buildings were designed with conservation as an objective: most of the design work would have occurred before the oil embargo. Energy use is higher for buildings in the 10-19 years old range, possibly due to a high level of use coupled with older, less efficient equipment. Energy use then tapers off to a low level for buildings through the 30-39 age range.. As buildings approach 40 years old the quality of the space significantly deteriorates. The building generally is in need of rennovation, and the space is perceived as being much less desirable than more modern space. Energy use declines due to less intensive use and inoperative equipment. Buildings in the $40+$ range have generally been renovated, and energy use rises, reflecting that the space is again high quality, prime space. Relatively low energy use in the $50+$ buildings probably is due to design practices of the 1920's, which resulted in buildings with good heat retention, and to the use of steam for heating.

1) Energy use was analyzed for downtown buildings to achieve compatibility with the available 1975 data. 
TABLE II-17

ENERGY CONSUMPTION ON A WEIGHTED BASIS BY BUILDING AGE, 1975 AND 1977 COMMERCIAL OFFICE BUILDINGS, DOWNTOWN BUILDINGS

Energy Use
$\left(10^{3}\right.$ Btu $)$

$$
\left(10^{3}\right. \text { Btu) }
$$

\section{Electric Use}

Btu/sq. ft., 1975

Btu/sq. ft., 1977

Other Fuels

Btu/sq. ft., 1975

Btu/sq: ft., 1977

Total Energy Use

Btu/sq. ft., 1975

No. of bldgs.

Btu/sq. ft., 1977

No. of bldgs.

\section{$1-9$}

99.2

97.1

51.8

64.2 .

$151: 0$
74

146.1

302
Building Age

$10-19$

20-29

30-39

40-49

$50+$

62.0

60.9

86.1

32.4

71.9

76.6

53.0

86.1

110.1

92.4

74.1

58.3

81.9

73.0

173.2

161.7

85.4

158.0

172.1

80

174.1

197

158.6

145.7

12

158.1

66

131.8

193 
TABLE II- $18 \mathrm{~A}$

ENEROY CONSUMPTION IN THE 1977 BOMA OFFICE

BUILOINO SAMPLE BY BUILDING AGE

DOWNTOWN BUILDINGS

WEIGHTED MEANS AND STANDARD DEVIATIONS

COMMERCIAL OFFICE BUILDINGS

$\begin{array}{cccccc}1-9 & 10-19 & 20-29 & 30-39 & 40-49 & 50+ \\ \text { YEARS } & \text { YEARS } & \text { YEARS } & \text { YEARS } & \text { YEAKS } & \text { YEARS }\end{array}$

ELECTHIC BTU/SO,FT. $(000)$

MEAN

STANDARD DEVIATION

UTHER FUELS BTU/SO.FT. 10008

MEAN

SIANDARD DEVIATIUN

IOTAL BTU/SO.FT. $(000)$

MEAN
STANDARD DEVIATION

TOTAL SQUARE FEET $(000,000)$

NUMBER OF BUILDINGS

97.1

87.3

86.1

87.7

3.1. 3

28.6

76.6

60.9

39.8

37.4

$64.2 \quad 92.4$

74.1

58.3

81.9

73.0

73.1

102.2

63.8

36.1

.45 .6

46.8

$\begin{array}{rrrrrr}146.1 & 174.1 & .158 .6 & 145.7 & 158.1 & 131.8 \\ 71.4 & 114.8 & 82.9 & 45.7 & 55.4 & 53.9 \\ 96.1 & 46.5 & 16.6 & 2.5 & 21.4 & 28.7 \\ 302.0 & 1.97 .0 & 72.0 & 12.0 & 66.0 & 193.0\end{array}$

TABLE II-18B

ENERGY CONSUMPTION IN THE 1977 BOMA OFFICE BUILDING SAMPLE BY BUILDING ASE DOWNTUWN BUILDINGS

UNWEIGHTED MEANS AND STANDARD DEVIATIONS COMMERCIAL OFFICE BUILDINGS

$\begin{array}{llll}1-9 & 10-19 & 20-29 & 30-39\end{array}$

YEARS YEARS YEARS YEARS

ELECTRIC BTU/SO.FT. (000)

MEAN
STANDARD DEVIATIUN

$$
98.3
$$

86.4

77.8

69.4

73.2

57.2

.40 .3

36.2

30.2

29.6

39.8

32.8

()THER FUELS BTU/SO.FT. (000)

MEAN
STANDARD DEVIATIUN

68.2

89.2

7.4 .9

60.1

77.9

66.6

73.8

94.5

60.9

37.9

50.4

40.1

TOTAL BTU/SO.FT. $(000)$

MEAN
STANDARD DEVIATION

TOTAL SOUARE FEET $(000,000)$

NUMBER OF BUILDINGS

$\begin{array}{rrrrrr}142.7 & 166.1 & 150.5 & 124.4 & 148.8 & 118.6 \\ 72.0 & 101.3 & 70.8 & 54.0 & 66.1 & 52.1 \\ 96.1 & 46.5 & 16.6 & 2.5 & 21.4 & 28.7 \\ 302.0 & 197.0 & 72.0 & 12.0 & 66.0 & 193.0\end{array}$




\section{II-6 Electric Billing Policy of Management}

Charges for electricity are usually included in the building rent, and an escalator clause typically provides for the pass-through of increased energy charges for a building in proportion to the space rented by each tenant. As Table II-19 indicates, electricity was included in the rent for 532 buildings in the 1977 data base. Since electricity is centrally metered with the charges distributed to the tenants on the basis of occupied space, the tenant typically has little control over his electric bill and little incentive to conserve energy (except in cases where the tenant occupies the entire building).

In 101 cases electricity was sold on a metered basis by the building to the tenant. Typically, the metering would be restricted to lighting and to those parts, if any, of the heating, ventilating, and air conditioning system on a separate circuit. On a metered basis the tenant should benefit from any conservation efforts he makes. The building electricity use reported for the 101 cases presented in Table 19 includes, according to BOMA, both electricity use billed to the tenant directly on the basis of metering and building electricity use for such general purposes as hallway lighting, space conditioning (if not billed to the tenant), and other uses not billed on the basis of metering.

In 24 cases electricity was sold by the building management to the tenant on an estimated basis; i.e., the building management and the tenant negotiated a mutually acceptable payment which, however, was not metered. This type of situation would typically occur when the tenant had installed equipment expected to use large amounts of electricity; the building management attempts to recover part of the cost of the abnormally high electric use through an estimated, projected additional charge. In this case a tenant's control over his electric bill is largely limited to the decision of whether to install the equipment. Usage of the equipment once it is installed will not have an appreciable effect on the bill. 
In 39 cases electric use was directly metered by the utility: rather than paying the landlord directly for electricity the tenant pays the utility. According to BOMA, all electric use for the building -- whether metered to the tenant or to the landlord - is included in the computed Btu/Sq. Ft. for electricity. The tenant can directly affect his electric bill and can benefit from conservation efforts in cases of direct metering.

In cases in which tenants were metered for their electric use and could benefit from conservation, they tended to conserve. 
TABLE II-19A

ELECTRIC BILL POLICY OF BUILDING MANAGEMENT IN

1977 BOMA OJFFICE BUILDING SAMPLE

WEIGHTED MEANS AND STANDARD DEVIATIONS

COMMERCIAL OFFICE BUILDINGS

ELECTRIC BTU/SO.FT.

MEAN (000)

STANDARD DEVIATION $(O \infty)$

SOUARE FEET $(000,000)$

NUMBER OF BUILDINGS

\section{SOLD \\ METERED}

SULD

RENT

88.3

83.3

101.3

64.8

88.7

36.9

38.5

21.6

31.6

44.1

133.0

27.1

6.9

10.2

34.6

.. 522.101.

24.

3.9 。

156.
TABLE II $-19 B$

ELECTRIC BILL POLICY OF BUILDING MANAGEMENT IN 1977 BOMA OFFICE BUILDING SAMPLE

UNWEIGHTED MEANS AND STANDARD DEV IATIUNS COMHERCIAL OFFICE BUILDINGS

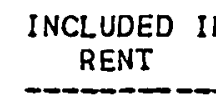

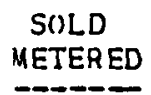

SOLD

ESTIMATED
METERED

BY UTILITY
No)

RESPONSE

ELECTRIC BTU/SO.FT.

MEAN (0OO)

STANDARD DEVIATION (OOO)

SOUARE FEET $(000,000)$

NUMBER OF BUILDINGS

$\begin{array}{lllll}84.3 & 70.8 & 77.0 & 73.3 & 84.3 \\ 40.5 & 34.4 & 39.2 & 38.1 & 40.8\end{array}$

133.0

27.1

0.9

10.2

34.6

522.

101.

24.

39.

156. 


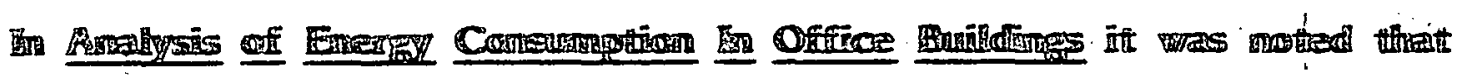

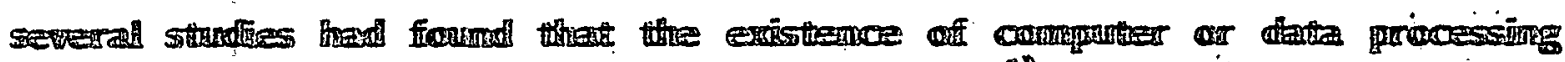

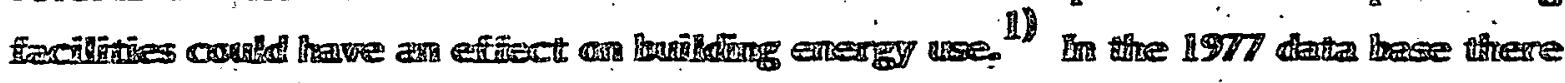

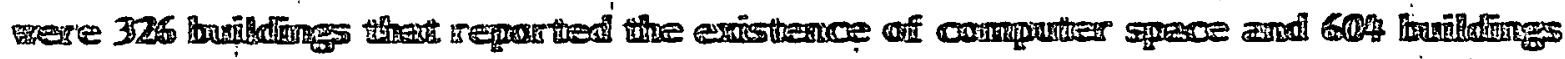

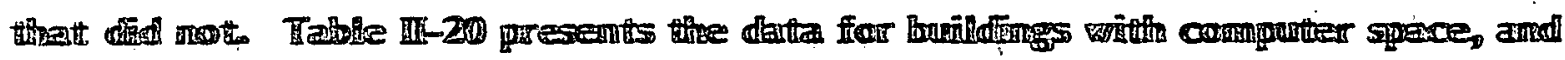

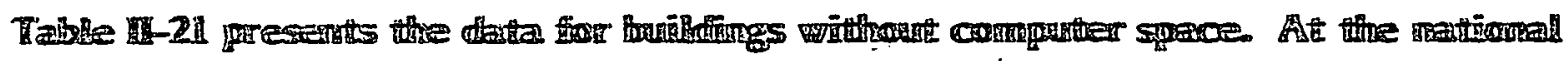

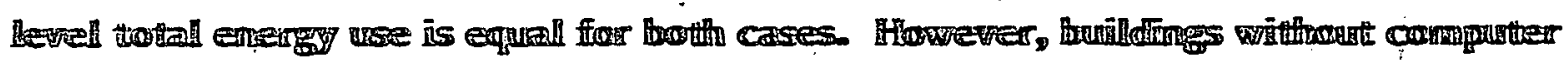

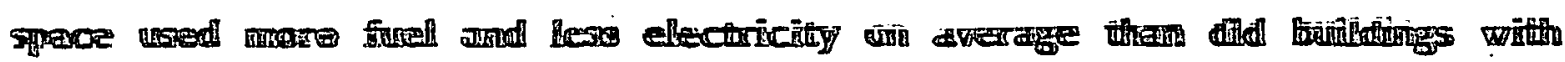
compros space.

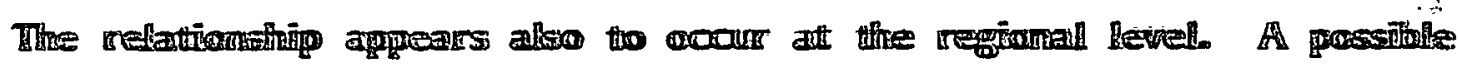

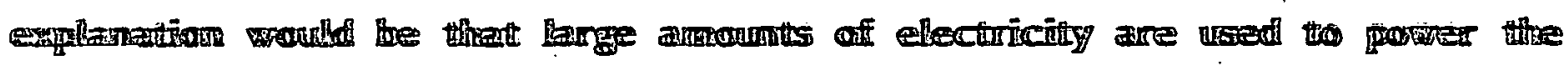

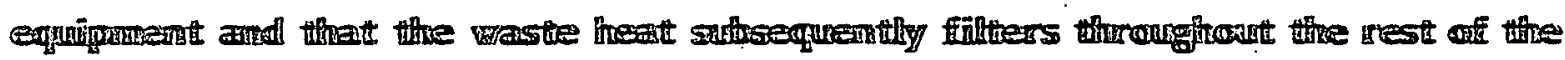

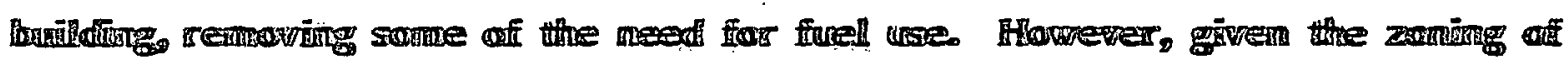

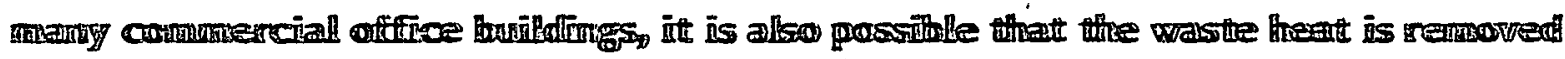

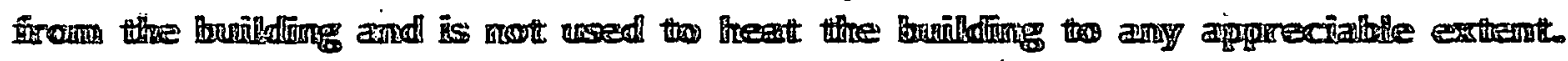

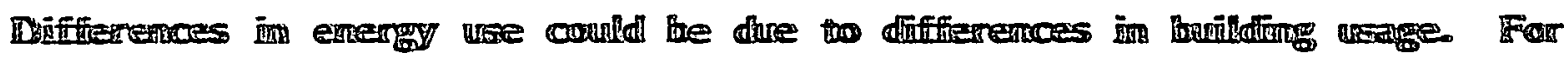

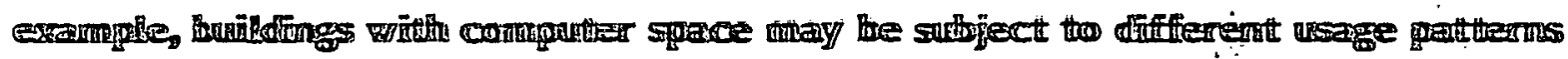

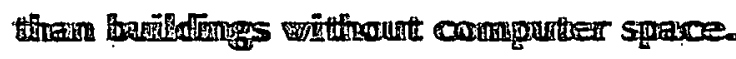

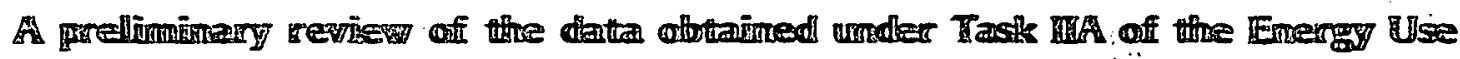

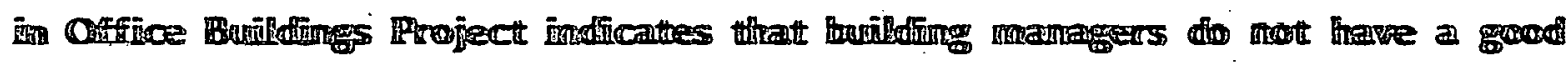

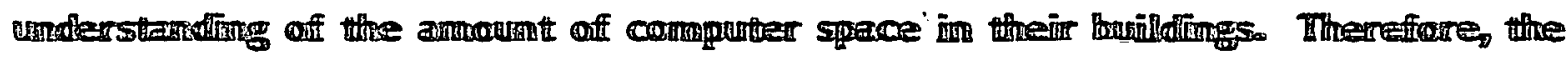

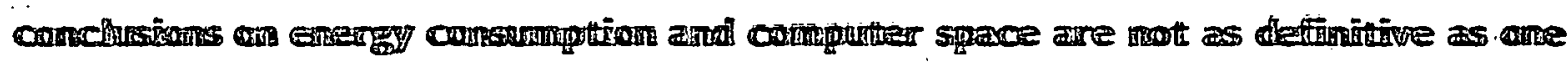

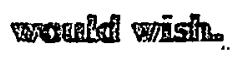

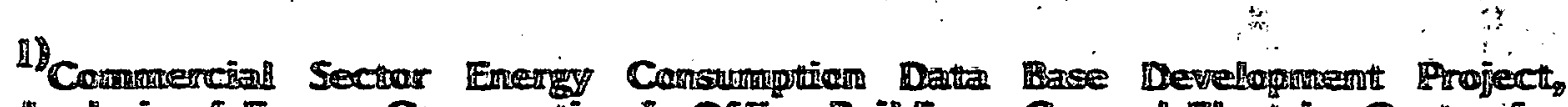

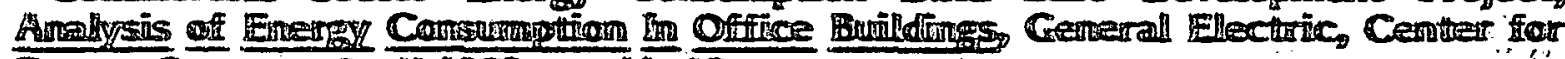

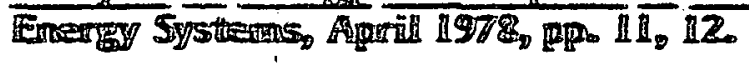




\section{TABLE II-20A}

ENERGY CONSUMPTION IN THE 1977 BOMA OFFICE BUILDING SAMPLE BUILDINGS WITH COMPUTER SPACE

(BTU/SO.FT. ${ }^{\infty}$ WEI
WEHTED UEANS AND STANDARD DEVIATIONS COMMERCIAL OFFICE BUILDINGS

COMPUTER

No.OFF FT: BLDG SO.FT/BLDG : ELECTRICITY
BLDGS (HILL $(000)$ (OOO)

MEAN STD.DEV.

TOTAL

NEW ENGLAND

MIDDLE ATLANTIC

EAST NORTH CENTRAL

32

781.7

13.6

$62.4 \cdot 14.9$

96.3

9.0

MEAN STD.DEV.

$\begin{array}{llll}49 & 25.1 & 512.8 & 11.3\end{array}$

$82.5 \quad 30.9$

66.2

37.1

158.7

11.1

$\begin{array}{llll}58 & 17.6 & 303.9 & 4.8\end{array}$

$74.2 \quad 31.3$

$87.3 \quad 49.8$

$148.6 \quad 38.0$

WEST NORTH CENTRAL

$82.4 \quad 30.7$

$99.7 \quad 123.4$

155.7 .59 .6

SOUTH ATLANTIC

EAST SOUTH CENTRAL

WEST SOUUTH CENTRAL

$\begin{array}{llll}52 & 11.5 & 220.9 & 0.2\end{array}$

$98.0 \quad 36.3$

$\begin{array}{llll}7 & 2.1 & 300.5 & 6.4\end{array}$

$116.0 \quad 18.2$

$86.7 \cdot 09.4$

$170.4 \quad 107.6$

MIIUNTAIN

$43 \quad 15.8 \quad 366.8 \quad 7.1$

123.249 .1

86.628 .2

$156.2 \cdot 73.8$

$\begin{array}{llll}15 & 3.5 & 231.5 & 24.9\end{array}$

104.151 .0

$53.5 \quad 00.2$

$173.5 \quad 60.2$

$\begin{array}{llll}53 & 1.9 .1 & 361.0 & 5.2\end{array}$

$53.4 \quad 68.0$

$164.9 \quad 80.0$

NATIUNAL AVERAGE

$\begin{array}{llll}326 & 107.9 & 330.9 & 7.3\end{array}$

$92.8 \quad 31.7$

46.1

56.6

149.7

72.5

$91.4 \quad 38.8$

71.0

06.6

131.5

62.9 


\section{TABLE II-20B}

ENERGY CONSUMPTION IN THE 1977 BOMA OFFICE BUILDING SAMPLE BUILDINGS WITH COMPUTER SPACE

UNWEIGHTED MEANS AND STANDARD DEVIATIONS COMMERCIAL OFFICE BUILDINGS

COMPUTER

\begin{tabular}{|c|c|c|c|c|c|c|c|c|c|}
\hline No.or & Sn. & $\begin{array}{l}\text { SOFT/ } \\
\text { BLDG }\end{array}$ & $\begin{array}{c}\text { SPACE } \\
\text { SO.FT/BLDG }\end{array}$ & ELECI & CRICITY & OTHER & A FUELS & & OTAL \\
\hline 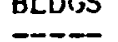 & (MILL) & -1000 & -1000 & MEAN & SIU.1DEV. & MEAN & SID.DEV. & MEAN & SIU.DEV. \\
\hline 3 & 2.3 & 781.7 & 13.6 & 65.4 & 16.4 & 100.6 & 13.2 & 166.1 & 18.5 \\
\hline 49 & 25.1 & 5.12 .8 & 11.3 & 81.5 & 34.1 & 60.6 & 31.1 & .142 .1 & 34.0 \\
\hline 58 & 17.6 & 303.9 & 4.8 & 76.6 & 34.7 & 93.9 & 03.4 & 160.0 & 74.8 \\
\hline 46 & $10: 8$ & 234.8 & 4.2 & 84.3 & 32.6 & .98 .4 & 97.2 & 172.0 & 89.6 \\
\hline $52^{\circ}$ & 11.5 & 220.9 & 6.2 & 94.0 & 38.8 & $\because 72.3$ & 57.1 & 140.6 & 63.4 \\
\hline 7 & $2 . i$. & 300.5 & 0.4 & 116.1 & 27.6 & 63.5 & 32.6 & 158.4 & 60.5 \\
\hline 43. & 15.8 & 366.8 & 7.1 & 121.6 & 53.8 & 53.5 & 47.6 & 162.1 & 78.4 \\
\hline 15 & $3.5^{\circ}$ & $231: 5$ & $24.9^{\circ}$ & 103.3 & 57.6 & 86.0 & $106: 2$ & 175.0 & 104.0 \\
\hline 53. & $19: 1$ & 361.0 & 5.2 & 92.4 & 38.6 & 56.1 & 74.5 & -135.0 & 78.8 \\
\hline 326 & 107.9 & 330.9 & 7.3 & 91.3 & $4 ! \cdot 5$ & .74 .8 & 08.5 & 152.8 & 73.4 \\
\hline
\end{tabular}

\section{NEW ENGLAND}

MIDOLE ATLANTIC

EAST NURTTH - CENTHAL

WEST NORTTH CENTHAL

SOUTH ATLANTIC

EAST ' SOUFH CENTRAL'

WEST. SOUTH CENTRAL

MOUNTAIN

PACIFIC.

NATIUNAL AVERAGE

$\begin{array}{llll}326 & 107.9 & 330.9 & 7.3\end{array}$

$91.3 \quad 4 ! \cdot 5$

.74 .8 
TABLE II - 2IA

ENERGY CONSUMPTION IN THE 1977 BOMA OFFICE BUILOING SAMPLE

HUILDINGS WITHOUT COMPUTER SPACE

WEIGHTED MEANS AND STANDARD DEVIATIONS

COMMERCIAL OFFICE BUILDINGS

\begin{tabular}{|c|c|c|c|c|c|c|c|c|c|}
\hline \multirow{2}{*}{ - } & \multirow{2}{*}{$\begin{array}{l}\text { No.OF } \\
\text { BLDGS }\end{array}$} & \multirow{2}{*}{$\begin{array}{c}\text { SH. } \\
\text { (MILL) }\end{array}$} & \multirow{2}{*}{$\begin{array}{l}\text { BLDG } \\
(000) \\
\end{array}$} & \multicolumn{2}{|c|}{ ELECTRICITY } & \multicolumn{2}{|c|}{ OTHEH FUELS } & \multicolumn{2}{|c|}{ TOTAL } \\
\hline & & & & MEAN & & MEAN & SIU.UEV. & MEAN & 310.1 \\
\hline NEW ENGLAND & 7 & 3.9 & 555.3 & 68.3 & 12.9 & 107.3 & 15.5 & 175.6 & 15.7 \\
\hline MIDDLE ATLANTIC & 59 & 22.9 & 388.6 & 72.0 & 18.5 & 78.2 & 34.6 & 150.2 & 45.0 \\
\hline EAST NORTH CENTHAL & 79 & 18.0 & 227.2 & 79.2 & 33.0 & 102.2 & 64.5 & 168.4 & 61.9 \\
\hline WEST NORTH CENTHAL & 58 & 9.4 & 161.8 & 79.9 & 37.4 & 85.6 & 45.2 & 150.8 & 62.5 \\
\hline SOUTH ATLANTIC & 136 & 22.9 & 1.68 .5 & 83.2 & 35.2 & 47.0 & 47.1 & 110.6 & 51.7 \\
\hline EAST SOUTH CENTHAL & 7 & 0.9 & 121.6 & 87.9 & $20: 1$ & 36.6 & $37: 3$ & 124.6 & 22.7 \\
\hline WEST SOUTH CENTHAL & 69 & $16: 9$ & 245.0 & .106 .2 & 45.9 & 96.0 & 155.7 & 194.8 & 172.8 \\
\hline - MOUNTAIN & 49 & 7.6 & 155.2 & 87.7 & .23 .7 & 106.9 & 121.1 & 191.6 & 130.2 \\
\hline PACIFIC : & 140 & 21.5 & 153.2 & 83.2 & 48.7 & 40.5 & 23.7 & 115.3 & 48.3 \\
\hline NATIONAL AVERAGE & 604 & 123.9 & 205.1 & 83.2 & 37.3 & 78.3 & 81.8 & 152.0 & 91.1 \\
\hline
\end{tabular}


TABLE II-21B

ENERGY CONSUMPTION IN THE 1977 BOMA OFFICE BUILDING SAMPLE BUILDINGS WITHOUT COMPUTER SPACE

UNWEIGHTED MEANS AND STANDARD DEVIATIONS

COMMERCIAL OFFICE BUILDINGS

NO.OF FT. SOFT/ BLDG $\quad$ ELECTRICITY OTHER FUELS

BLDGS (MILL) (OOO) MEAN STU.DEV. MEAN STU.DEV. MEAN STU.DEV.

HEW ENGLAND

MIDDLE ATLANTIC

$7 \quad 3.9 \quad 555.3$

67.0
13.3

105.2

17.9

172.2

18.3

EAST NORTH CENTRAL

$\begin{array}{lll}59 & 22.9 \quad 388.6\end{array}$

$70.5 \quad 29.1$

$79.0 \cdot 37.8$

1.48 .0

46.7

$\begin{array}{lll}79 & 18.0 \quad 227.2\end{array}$

$69.1 \quad .35 .3$

95.452 .1

155.5

58.9

WEST NORTH CENTRAL

$\begin{array}{lll}58 & 9.4 & 161.8\end{array}$

$76.7 \quad 37.8$

$90.9 \quad 47.0$

63.0

SOUIH ATLANTIC

$136 \quad 22.9 \quad 168.5$

$82.8 \quad 32.6$

$61.2 \quad 61.1$

1.24 .1

57.2

EAST SOUTH CENTKAL

$\begin{array}{lll}7 & 0.9 & 121.6\end{array}$

$78.5 \quad 23.5$

43.2

44.4 .

. 121.8

30.6

WEST SOUTH CENTKAL

$69 \quad 16.9 \quad 245.0$

$97.842 .5^{\circ}$

$87.5 \quad 135.7$

171.8

142.3

MOUNTAIN

PACIFIC

NATIUNAL AVEHAGE

$49 \quad 7.6 \quad .155 .2$

72.6

25.7

91.4 .96 .5

158.0

105.1

$140 \quad 21.5 \quad .153 .2$

70.9

46.3

$44.8 \quad 26.5$

105.7

50.3

604123.9205 .1

77.0

38.2

74.3

70.9

138.6

78.5 


\section{II-8 Energy Consumption and Building Air Conditioning}

Energy use as a function of type of building air conditioning is presented in Tables II-22 through II-25. There were 610 buildings identified as having electric air conditioning, 55 buildings with steam absorption, 12 buildings with steam turbine, and 8 with gas air conditioning. Electricity consumption was significantly lower for buildings with non-electric air conditioning.

Electric air conditioning occurred throughout the country. Steam air conditioning occurred to some degree in areas with steam plants. Although such air conditioning is less efficient than electric and can be more costly to operate, a major advantage of district steam is the elimination of much of the space requirements for space conditioning equipment in the building. Since building space is valuable in large cities, building managements in the past have viewed such a tradeoff as desirable. The limited amount of gas air conditioning occurred in the West South Central region; natural gas was abundant and relatively cheap in that region.

Chilled water is purchased for air conditioning by a number of buildings in major cities. The water is furnished from a chilling plant at a specific temperature and is then piped through the building and returned to the chilling plant at a higher temperature. The data furnished by building managements for chilled water use appeared in many cases to be inaccurate or uncertain; and, in addition, a conversion factor to obtain Btu's was unavailable. The conversion to Btu's would depend on the entry and exit temperatures of the water, and these numbers were not reported by the building managers to BOMA. Accordingly, buildings utilizing chilled water for air conditioning were dropped from the data base. Approximately 40 buildings were eliminated. 
TABLE II-22A

ENERGY CONSUMPIIUN IN THE 1977 BOMA OFFICE BUILUING SAMPLE BUILDINOS WITH ELECTRIC AIR CONDITIONING

(BTU/SO.FT. ODO)

WEIGHTED REANS AND STANDARD DEVIATIONS

\begin{tabular}{|c|c|c|c|c|c|c|}
\hline El & RICIIY & & . OTH & ER · FUELS & & TAL \\
\hline $\begin{array}{l}\text { No. . (OF } \\
\text { BLDGS }\end{array}$ & MEAN & STD.DEV. & MEAN & STD.DEV. & MEAN & STD.DEV. \\
\hline 6. & 61.4 & 12.6 & 101.8 & 11.2 & 163.1 & 17.0 \\
\hline 58. & 82.3 & 28.9 & 53.5 & 28.4 & 135.8 & 29.5 \\
\hline 105. & 80.7 & 33.1 & 86.5 & 45.3 & 157.0 & 50.0 \\
\hline 75. & 85.8 & 32.9 & 74.7 & 43.7 & $152: 9$ & 55.8 \\
\hline 95. & 92.4 & 33.5 & 49.7 & 3.1 .0 & 122.0 & 42.4 \\
\hline 12. & 109.1 & 22.3 & 70.1 & 39.4 & 101.4 & 57.5 \\
\hline 85. & 11.3 .6 & 45.7: & 60.3 & 115.5 & 164.1 & 124.7 \\
\hline 41. & 94.1 & 37.8 & $48: 6$ & 43.0 & 139.1 & .53 .6 \\
\hline 133. & 92.8 & 39.4 & 40.1 & 31.9 & 124.8 & 47.1 \\
\hline 6. & .91 .5 & 38.0 & 60.8 & 60.0 & 14.3 .0 & 67.6 \\
\hline
\end{tabular}

TABLE II- 228

ENERGY CONSUMPTION IN THE 1977 BOMA OFFICE BUILOING SAMPLE BUILDINGS WITH ELECTRIC AIR CONDITIONING (BTU/SO.FT. OOO) UNWEIGHTED MEANS AND STANDARD DEVIATIONS

MIDDLE ATLANTIC

EAST NORTH CENTRAL

WEST NORTH CENTRAL

SOUTH ATLANTIC

EAST SUUTH CENTRAL

WEST SUUTH CENTRAL

MOUNTAIN

PACIFIC

NATIONAL AVERAGE
COMMERCIAL OFFICE BUILDINGS
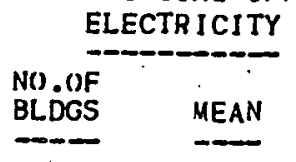

NEN ENGLAND

MIDDLE ATLANTIC

EAST NOPTH CENTRAL

WEST NORTH CENTRAL

ȘUUTH ATLANTIC

EAST SOUTH CENTRAL

WEST SOUTH CENTRAL

MUUNTAIN

PACIFIC

NATIOHAL AVERAGE

$\begin{array}{rrr}6 . & 60.4 & 10.9 \\ 58 . & 79.1 & 32.0 \\ 105 . & 72.3 & 35.4 \\ 75 . & 82.3 & 35.3 \\ 95 . & 89.7 & 32.7 \\ 12 . & .97 .3 & 31.4 \\ 85 . & 107.3 & 46.3 \\ 41 . & 82.5 & 39.4 \\ 133 . & 84.2 & 29.5 \\ 610 . & .85 .5 & 38.6\end{array}$

TOTAL

MEAN STD.DEV.

$167.7 \cdot 18.9$

$137.7 \quad 30.1$

$150.9 \quad 53.8$

$157.0 \quad 55.6$

$124.0 \quad 51.6$

$140.1 \quad 49.5$

$\begin{array}{llll}53.4 & 87.9 & .150 .0 & 100.1\end{array}$

$61.6 \quad 42.4,138.1,51.7$

$\begin{array}{llll}43.3 & 27.9 & 117.4 & 43.3\end{array}$

$\begin{array}{llll}64.6 & 50.8 & 137.9 & 60.0\end{array}$ 
TABLE II - 23 A

ENERGY CONSUMPTION IN THE 1977 BOIMA OFFICE BUILDING SAMPLE BUILDINGS WITH GAS AIR CONOITIONING

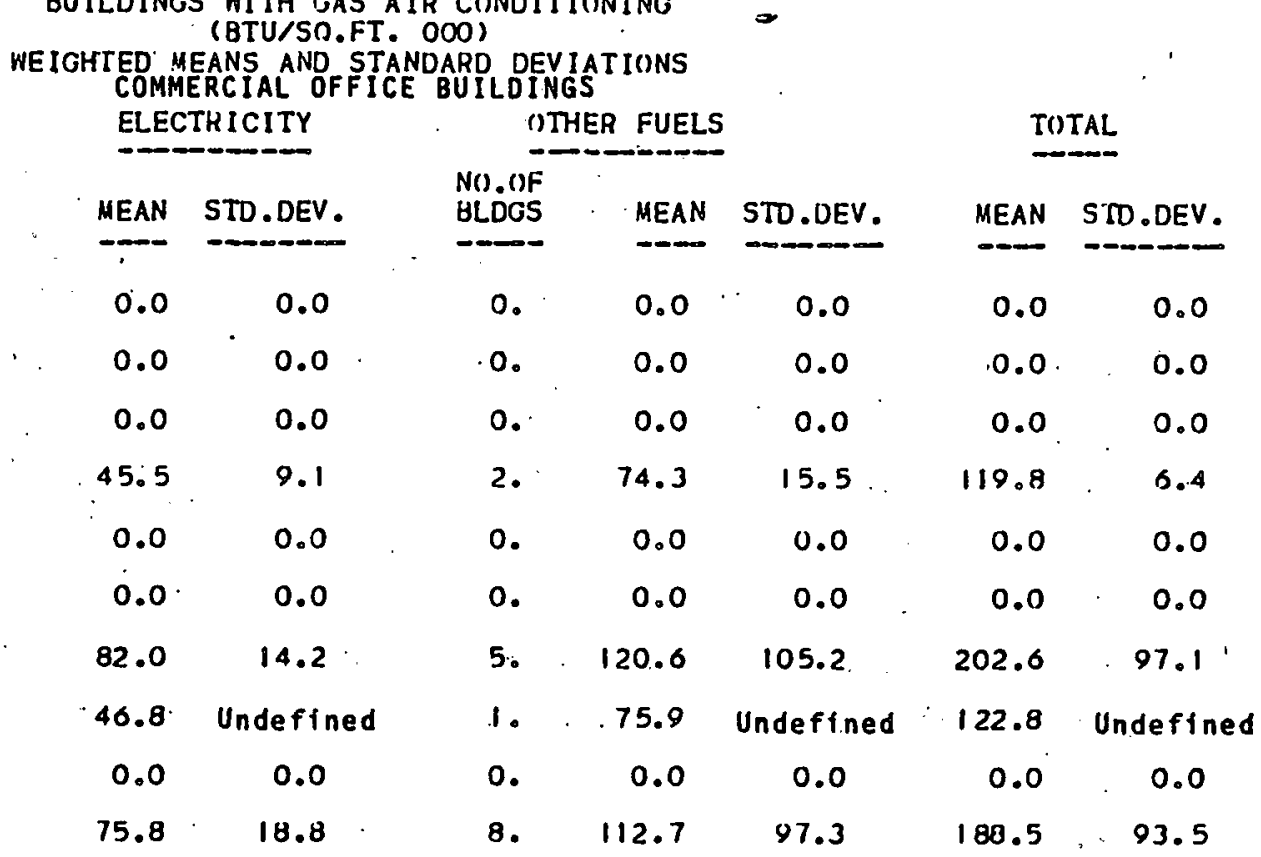

TABLE 11-23B

ENERGY CONSUMPTION IN THE 1977 BOMA UFFICE BUILDING SAMPLE BUILDINGS WITH GAS AIR CONDITIONING (BTU/SO.FT, OOO) UNWEIGHTED MEANS AND STANDARD DEVIATIONS COMMERCIAL OFFICE BUILDINGS

ELECTRICITY

MEAN STD.DEV.

'NEW ENGLAND

MIDDLE ATLANTIC

EAST NORTH CENTRAL

WEST NORTH CENTRAL

SOUTH ATLANTIC

EAST SOUTH CENTRAL

WEST SUUTH CENTRAL

KOUNTAIN

PACIFIC

NATIONAL AVERAGE

0.0

0.0

0.0

0.0

0.0

$47.8 \quad 13.3$

0.0 . 0.0

0.0

0.0

$77.4 \quad 14.2$

46.8

0.0

0.0

0.0

66.2

19.5

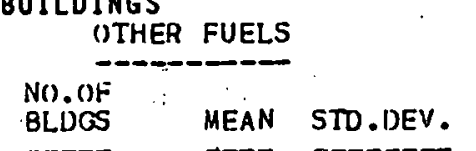

0.

0.0

0.0

0.

0.0

0.0

0 .

0.0

0.0

2. 70.4

22.6

0.

0.0

0.0

0.

0.0

0.0

5. $\quad 215.4 \cdot 192.9$

1. $\quad 75.9$

0.0

0.

0.0

0.0

B. $161.7 \quad 163.8$
TOTAL

MEAN STD.DEV 。

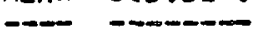

0.0

0.0

0.0

0.0

0.0

0.0

$118.2 \quad 9.3$

$0.0 \quad 0.0$

$0.0 \quad 0.0$

$292.8 \cdot 184.9$

$122.8 \cdot 0.0$

$0.0 \quad 0.0$

$227.9 \quad 166.1$ 
TABLE II-24A

ENERGY CONSURPTION IN THE 1977 BOMA OFFIICE BUILDING, SAMPLE BUILDINGS INITH STEAM ABSORPTION AIR CONDITIIONING WEIGHTED MEANS (BTU/SN.FT. ODO)

\begin{tabular}{|c|c|c|c|c|c|c|c|}
\hline$\because$ & $\begin{array}{l}\text { WEIGHTED M } \\
\text { COMM } \\
\quad \text { ELEC }\end{array}$ & $\begin{array}{l}\text { ERTU/SO. } \\
\text { EANS AND S S } \\
\text { ERIAL OFF } \\
\text { TRICITY. }\end{array}$ & $\begin{array}{l}\text { OT DEV } \\
\text { UILDIN }\end{array}$ & $\begin{array}{l}\text { TIONS } \\
\text { R FUTELS } \\
\end{array}$ & & & TAL \\
\hline & MEAN & STD,DEV. & $\begin{array}{l}\text { NO).OFF } \\
\text { BLDGS. }\end{array}$ & MEAN & STD.DEV. & MEAN & STD.DEV. \\
\hline NEI ENGLAND & 69.8 & 13.8 & 4. & 104.2 & 16.4 & $\because 174.0$ & 14.1 \\
\hline MIDULE ATLANTIC & 69.3 & 16.1 & 14. & 91.2 & 31.9 & $\because 160.4$ & 42.6 \\
\hline EAST NURTH CENTIRAL & 70.6 & 18.1 & 8. & 153.2 & 54.4 & $\because 223.8$ & 52.3 \\
\hline WEST NORTH CENTRAL & 56.0 & 21.5 & 11. & 153.7 & 174.8 & 203.7 & 161.5 \\
\hline SOUTH ATLANTIC & 74.8 & 23.1 & 8. & .157 .6 & 84.3 & 232.4 & 105.4 \\
\hline EAST SOUTH CENTRAL & 0.0 & 0.0 & 0. & 0.0 & 0.0 & 0.0 & 0.0 \\
\hline WEST SOUTH CENTRAL & 127.1 & 74.5 & 3. & 268.7 & 153.2 & 3.95 .8 & 224.7 \\
\hline MOUNTAIN & 96.2 & 19.0 & 3. & 290.3 & 83.0 & 386.5 & 101.5 \\
\hline PACIFIC & 62.3. & 24.4 & 5. & 139.7 & 127.3 & 20.1 .9 & 148.5 \\
\hline NATIONAL AVERAOE & 70.0 & 23.5 & 56. & 129.4 & 101.0 & 198.2 & 108.3 \\
\hline
\end{tabular}

TABLE II-2.4B

ENEROY CONSUMPTION IN THE 1977 BUIMA OFFICE BUILOINO SAMPLE BUILDINCS WITH STEAM ABSORPTION AIR CONDITIONINO (BTU/SO.FT. ODO) UNWEIGHTED MEANS AND STANOARD DEV IATIONS COMMERCIAL OFFICE BUILDINGS

NEW ENGLAND

MIDDLE ATLANTIC

EAST NURTH CENTRAL

WEST NORTH CENTHAL

SOUTH ATLANTIC

EAST SUUTH CENTRAL

WEST SIUUTH CENTRAL

MOUNTAIN

PACIFIC

NATIONAL AVERAG:E

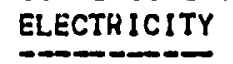

MEAN STD.DEV.

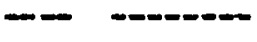

$60.7 \quad 18.4$.

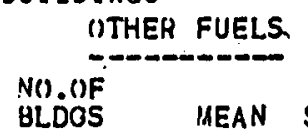

$68.4 \therefore 23.4$

$67.8 \quad 22.0$

60.427 .1$.

$67.4 \quad 20.0$

$0.0 \quad 0.0$

$112.0 \quad 86.0$

$88.5 \quad 22.5$

$69.3 \quad 37.8$,

$70.1 \quad 30.4$

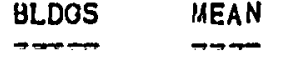

4.

107.6

95.5

8. 159.8

11. 178.5

8. 135.3

0.0

3. 248.1

3. $\quad 280.0$

182.8

114.9

5. 174.5

56. .152 .6

159.5

111.9

40.1

48.7

164.5

77.0
TUTAL

MEAN STD.DEV,

174.3

17.2

163.9

57.4

227.5

50.1

233.4

155.2

202.

94.4

0.0

0.0

$360.0 \quad 263.9$

$368.6 \quad 135.8$

$243.8 \quad 194.0$

221.5

126.2 
TABLE I]-25A

ENERGY CONSUMPTION IN THE 1977 BOMA OFFICE BUILDING SAMPLE BUILDINGS WITH STEAM TURBINE AIR CONDITIONING (BTU/SO.FT. OOO) WEIGHTED MEANS AND STANDARD DEVIATIONS
COMMERCIAL OFFICE BUILDINGS

ELECTRICITY

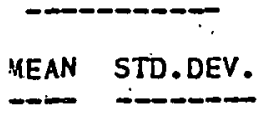

$0.0 \quad 0.0$

$73.6 \quad 25.5$

MIIDOLE ATLANTIC

EAST NORTH CENTRAL

WEST NOHTH CENTRAL

SIUTH ATLANTIC

EAST SOUTH CENTRAL

WEST SOUTH CENTRAL

M()UNTAIN

PACIFIC

NATIONAL AVERAGE

$50.6 \quad 15.0$

0.0

0.0

0.0

0.0

0.0

0.0

60.6 Undefined

$0.0 \quad 0.0$

0.0

0.0

70.8

.25 .4
(ITHER FUELS

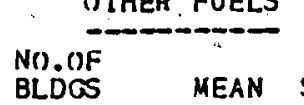

STU . DEV .

0.0

0.0

9. $\quad 103.6$

23.7

2.

92.2

129.9

0.0

0.0

0.0

0.0

0.0

0.0

0.0

0.

258.8

Undefined

319.4

0.0

Undefinec

o.

0.0

0.0

0.0

0.0

O.

0.0

0.0

12. 104.0

52.0

174.8
0.0

67.2

TABLE II -25B

ENERGY CUNSUMPTION IN THE 1977 BOMA IFFICE BUILDING SAMPLE BUILDINGS WITH STEAM TURBINE AIR CONDITIONING (BTU/SO.FT. ODO) UNWEIGHTED MEANS AND STANDARD DEVIATIINS COMMERCIAL OFFICE BUILOINGS

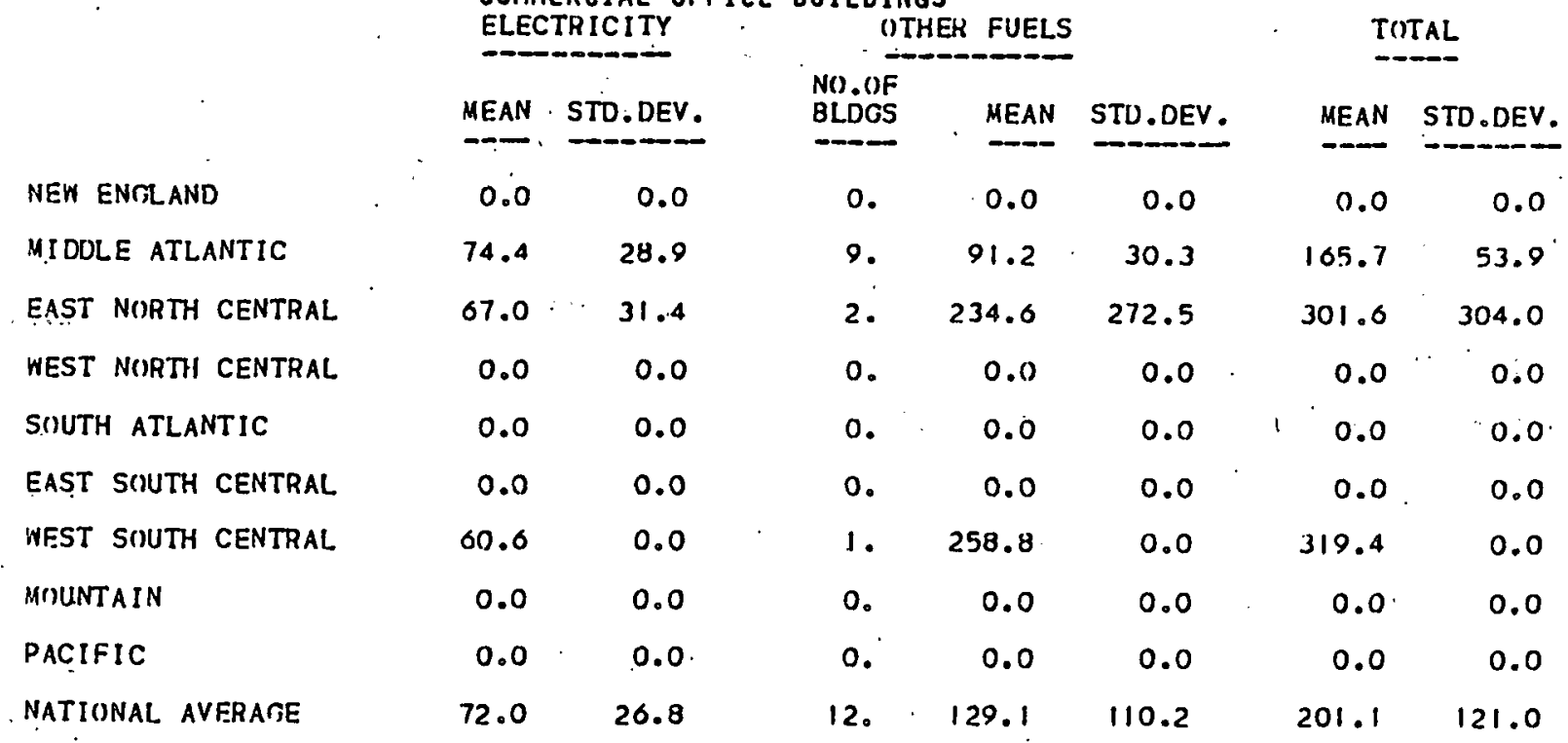


The 1977 data base developed from the 1977 BOMA Experience Exchange Report was generally representative of office buildings in terms of location and age. No data are available to classify buildings in terms of prime and non-prime space; it is, therefore; impossible to determine to what degree the 1977 data base was representative in terms of quality of space. It is believed, however, that the commercial office buildings represented in the Experience Exchange Report are better managed than are office buildings in general. Therefore, it is likely that the 1977 data base is unrepresentative of office buildings in general in terms of the prime/non-prime classification.

The tables developed summarize energy use but do not provide explanations of the causes of the variation in energy use by fuel type, building usage, or location. In presenting the major conclusions on building energy use we will offer some comments on variations in "energy use; these explanations 'were' developed on the basis of discussions with the BOMA staff. A regression analysis will be developed under Task IC of this project in an attempt to explain some of the energy consumption variations in terms of building characteristics and use.

- Electrically and steam heated buildings use less energy than do buildings heated with gas and oil.

- In the cases of gas, oil, and steam heated buildings, electric use per square foot is greater for gas than for oil and steam buildings. It is not known why there is this variation. One possibility is that the variation is related to the heating, ventilating, air conditioning system configuration and to the use of energy for reheat.

o. Both electric and fuel use are related to climate.

- Downtown buildings are less energy efficient than are suburban buildings in the case of oil and gas heated buildings. There is however, no difference for electric and steam heated buildings. 
- Buildings 10 stories or higher tend to use more electricity than do lower buildings. This is probably due to increased elevator and air handling requirements. There is no clear trend in terms of fuel use by building height.

- Energy use is a function of building age. Middle aged buildings -- most likely to be approaching the non-prime space category -- use less energy than do younger buildings or older buildings, which have in general been renovated.

- Building electric use is lower in cases in which the tenant is metered for his use.

- The presence of computer space has an impact on building energy consumption. Buildings with computer space use more electricity but less fuel than do buildings without computer space; the overall level of energy is, however, the same between the two types of buildings.

Most buildings air condition with electricity. In cities with district steam there is some steam air conditioning. Although the operating cost of steam air conditioning is more expensive than that of electric air conditioning, buildings which heat and cool with steam are able to utilize space for commercial purposes which would otherwise be devoted to boiler rooms. In some cities such a space/cost tradeoff has been advantageous. In addition, the choice of steam where available relieves the building manager of fuel availability and handling problems. 
The 1977 BOMA Experience Exchange Report contained data for 233 Federal, state, and local government office buildings." Government office buildings were " defined to be office buildings owned and operated by a government agency. Buildings privately owned and leased to the government were classified elsewhere as commercial buildings. After the data had been subjected to a verification and correction process identical to that for the commercial office buildings, there were 216 government office buildings which remained in the 1977 data base.

The government buildings in the 1977 data base were not typical of government buildings in general either in terms of location or in terms of age. The regional distribution of government administration buildings in 1975 was computed on the basis of data in Commercial Buildings Inventory. 1) As is indicated in Table III-1 the square feet of government office space in the 1977 data base are not distributed in a pattern similar to the overall distribution of all government administration space in 1975 (data for the 1977 census division distribution of space have not been developed). Table III-2 presents the age distribution of government office space in 1977; in comparison, the buildings in the 1977 data base are under represented in the 1-9, 20-29, and 50+ age categories, and are over represented in the 10-19 and 40-49 categories. The age distribution of the government buildings in the 1977 data base in comparison to the age distribution of government administration buildings in general is not as bad as the geographic distribution.

The government buildings in the 1977 data base can be classified in terms of the type of fuel used for heating: electric, gas, oil, and steam.

$\begin{array}{lcccc}\begin{array}{c}\text { Building Fuel } \\ \text { Type }\end{array} & \begin{array}{c}\text { Number of } \\ \text { Buildings }\end{array} & \begin{array}{c}\text { \% of } \\ \text { Buildings } \\ \text { Electric }\end{array} & \begin{array}{c}\text { Square Feet of } \\ \text { Space }\left(10^{6}\right)\end{array} & \begin{array}{c}\% \text { of } \\ \text { Space }\end{array} \\ \text { Gas } & 8 & 3.7 \% & 2.4 & 4.6 \% \\ \text { Oil } & 124 & 57.4 & 19.0 & 36.3 \\ \text { Steam } & 26 & 12.0 & 4.1 & 7.8 \\ & 58 & 26.9 & 26.8 & 51.2\end{array}$

1) General Electric Company, Center for Energy Systems, Commercial Buildings Inventory, June.1978. 
Table III-3 describes the government buildings in the 1977 data base by fuel type and census division. There are relatively few electric buildings, and the buildings are significantly larger than the gas or oil heated buildings. There are 124 gas heated buildings, and most census divisions have a sample of at least 10 buildings. The 26 oil heated buildings are relatively evenly distributed among census divisions, and the steam heated buildings are predominantly located in the East North Central and West North Central divisions. In comparison to the commercial office buildings (Table II-3) the gas and oil heated government office buildings are smaller, and the steam heated buildings are larger.

Table IIl-4 tabulates the building inventory by age. In contrast to commercial office buildings in the 1977 data base, government buildings tend to fall in the middle age ranges; there are fewer old $(50+)$ or young (1-9) buildings.

$\begin{array}{ccc}\ldots & \begin{array}{c}\text { Percent of Buildings in Age Category } \\ \text { (By Square Feet) }\end{array} \\ \therefore & \text { Commercial } & \\ \text { Age : } & & \text { Government } \\ 1-9 & 45.7 \% & 26.0 \\ 10-19 & 21.8 & 37.7 \\ 20-29 & 7.8 & 6.5 \\ 30-39 & 1.0 & 7.3 \\ 40-49 & 9.6 & 13.0 \\ 50+ & 14.0 & 9.6\end{array}$




\section{TABLE III-1}

Percentage Distribution of Square Feet of Government Office Building Space by Census Division

\section{Estimated Distribution of \\ Government Office Byjlding Space for 1975 \\ (Percent of Total Space by Region)}

Census Division
Distribution of Square Feet of Government Spage in 1977 Data Base

(Percent of Sample)

3.2

12.1

3.2

23.7

4.2

8.9

14.2

20.5

10.0

1) Based on information in Commercial Buildings Inventory, General Electric, Center for Energy Systems, June 1978; includes federal, state, and local space.

2) Based on information obtained from BOMA; includes federal, state, and local space. 
Estimated Distribution of Square Feet of Government Office Building Space by Age in 1977

Age

$1-9$

$10-19$

20-29

$30-39$

$40-49$

$50+$
$32.8 \%$

28.0

10.3

7.4

9.1

12.4
Distribution of Square Feet of Government Building Space in 1977 Data Base ${ }^{2 \text { ) }}$ (Percent of Sample)

$26.0 \%$

37.7

6.5

7.3

13.0

9.6

1) Based on information developed in Commercial Building's Inventory, General Electric, Center for Energy Systems, June 1978.

2) Does not țotal $100 \%$ due to rounding. 
Table III-5 compares energy use between commercial and government of fice buildings. With the exception of steam heated buildings, government office buildings use less energy than do commercial office buildings; discussions with BOMA suggested that differences in building construction and building usage could account for this difference in energy consumption. The government architectural style results in building design which is structurally more massive than commercial space and probably has better thermal characteristics. In addition, building managers for government buildings have frequently received directions to operate the buildings at comfort levels for lighting, heating; and cooling which are below those frequently maintained in the private sector. Commercial building managers in providing space conditioning have to maintain a level of tenant satisfaction and comfort that will enhance the marketability of the space. In contrast, government building managers operate the buildings pursuant to the instructions resulting from government conservation policies. The immediate satisfaction of the building tenants is of less importance than is the case in the commercial sector.

Table III-6 summarizes government office building energy use on a regional basis, and Tables III-7 through III-11 provide detailed statistical information by census division. Table III-7 provides the data for all buildings combined by fuel type. Table III- 8 provides data for electrically heated buildings; since the number of buildings is very limited it is probably not appropriate to use these data at other than the national level. Table III-9 presents data for gas heated buildings. In comparison to commercial of fice buidings (Table II-10), fuel use is generally less in all census divisions. In some divisions (Middle Atlantic, sample size 6; South Atlantic, sample size 8; West South Central, sample size 15; Mountain, sample size 30) it is significantly lower and, in view of the adequate sample sizes, the deviation is probably due to factors other than random statistical error. For gas heated buildings the electric use is also lower than is the case for commercial buildings, with particularly dramatic reductions in the case of the South Atlantic and Pacific divisions. 
Table III-10 presents data for oil heated buildings. In comparison to commercial office buildings (Table II-I1) government office buildings used more electricity but less oil. It is not known why this is the case. A possible explanation might, however, be that the buildings are major regional government installations with data processing and/or other high usage applications.

Table III-11 presents the data for steam heated buildings. In comparison to commercial office buildings (Table II-12) government office buildings use slightly less electricity but more steam. Higher steam use is particularly noticeable in the cases of West North Central, South Atlantic, and Pacific. Again, the causes of the higher use are unknown. 
TABLE III - 3

THE 1977 BOMA OFFICE BUILDING SAMPLE

BY TYPE OF IIEATING FUEL AND REGION

(SOUARE EEET NN MILLIONS)
GOVERMENT OFFICE BUILOINGS

ELECTHIC

GAS
$\begin{array}{ccc}\text { NUMBEH } & \text { SO. } & \text { SOFT/BLDG } \\ \text { OF BLDGS } & \text { FT. } & (000)\end{array}$

NUMBER
SOF BLDGS
FT.

OIL

$\begin{array}{ccc}\text { NUMBEH } & \text { SN. SOFT/8LDG } \\ \text { UF BLDGS } & \text { FT. } & (000)\end{array}$

STEAM

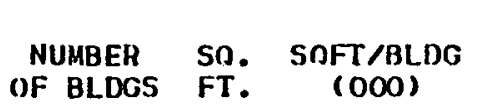

\section{NEW ENGLAHD}

MIDDLE ATLANTIC

EAST NIIRTH CENTRAL

WEST NOHTH CENTHAL. SIUTH ATLANTIC

FAST SIUTH CENTHAL

WEST SIUUTH CENTRAL

NOUNTAIN

PACIFIC

TOTAL

$\begin{array}{rrrr}676.2 & 4 & 0.6 & 152.3 \\ 290.5 & 6 & 2.3 & 380.0 \\ 411.6 & 11 & 0.6 & 53.3 \\ 148.1 & 23 & 4.5 & 197.2 \\ 0.0 & 8 & 0.8 & 101.6 \\ 0.0 & 10 & 1.17 & 173.9 \\ 0.0 & 18 & 2.7 & 149.9 \\ 232.4 & 30 & 3.9 & 129.1 \\ 116.7 & 14 & 1.9 & 136.2 \\ -1 .-1 & -1 & --1 & --1.2\end{array}$

301.6

$.124 \quad 19.0$

153.6

2

0.129 .5

3

0.9

287.2

1. 0.3 .290 .5

30.6 .194 .9

$4 \quad 0.1 \quad 35.0$

$5 \quad 1.2 \quad 244.0$

31.4

483.0

$0 \quad 0.0$

51.3

262.3

7

2.

305.4

$10 \quad 4.4 \quad 436.1$

$29 \quad 16.2 \quad .560 .0$

$10.3 \quad 347.6$

$0 \quad 0.0$

0.0

$00.0 \quad 0.0$

$\begin{array}{lll}0 & 0.0 & 0.0\end{array}$

$20.1 \quad 59.0$

1

0.6010 .0

$4 \quad 0.3 \quad 72.6$

51.

223.1

$-$

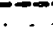

.156 .6 
TABLE 111 - 4

HEATINS FUEL BY AGE OF BUILDING IN THE 1977 BOMA OFFICE BUILDING SAMPLE

(SOUARE FEET IN MILIONS)

GOVERNMENT OFFICE BUIL.DINGS

HEATING FUEL

\begin{tabular}{|c|c|c|c|c|c|c|c|c|c|c|c|c|c|c|c|}
\hline \multirow[b]{2}{*}{$\begin{array}{l}\text { AGE } \\
\text { (YEARS }\end{array}$} & \multicolumn{3}{|c|}{ ELECTHIC } & \multicolumn{3}{|c|}{ GAS } & \multicolumn{3}{|c|}{ OIL } & \multicolumn{3}{|c|}{ STEAM } & \multicolumn{3}{|c|}{ TOTAL } \\
\hline & $\begin{array}{l}\text { NO.OF } \\
\text { BLDGSS }\end{array}$ & $\begin{array}{l}\text { SO. } \\
\text { FT. }\end{array}$ & $\begin{array}{c}\text { So.FT./ } \\
\text { BLDG } \\
(000)\end{array}$ & $\begin{array}{l}\text { NO).OFF } \\
\text { BLDGSS }\end{array}$ & $\begin{array}{l}\text { so. } \\
\text { FT. }\end{array}$ & $\begin{array}{c}\text { SO.FT./ } \\
\text { BLDG } \\
(000)\end{array}$ & $\begin{array}{l}\text { NO.OFF } \\
\text { BLDGS }\end{array}$ & $\begin{array}{l}\text { so. } \\
\text { FT. }\end{array}$ & $\begin{array}{l}\text { So.FT./ } \\
\text { BLDG } \\
(000)\end{array}$ & $\begin{array}{l}\text { NO.OF } \\
\text { BLDSSS }\end{array}$ & $\begin{array}{l}\text { So. } \\
\text { FT. }\end{array}$ & $\begin{array}{c}\text { SO.FT./ } \\
\text { BLDG } \\
(000)\end{array}$ & $\begin{array}{l}\text { NO..iF } \\
\text { BLDGS }\end{array}$ & $\begin{array}{l}\text { SO: } \\
\text { FT: }\end{array}$ & $\begin{array}{c}\text { So.FT.' } \\
\text { BLDG } \\
\text { (OOO) }\end{array}$ \\
\hline $1-9$ & 4 & 1.4 & 338.8 & 27 & 5.3 & 197.0 & 7 & 1.1 & 150.2 & 11 & 5.9 & 532.8 & 49 & 13.6 & 277.3 \\
\hline $10-19$ & 3 & 0.9 & 303.2 & 35 & 9.3 & 266.1 & 6 & 1.2 & 194.9 & 17 & 8.3 & 488.0 & 61 & 19.7 & 322.8 \\
\hline $20-29$ & 0 & 0.0 & 0.0 & $B$ & 1.8 & 230.6 & 2 & 0.2 & 116.8 & 1 & 1.3 & 1316.6 & 11 & 3.4 & 308.6 \\
\hline $30-39$ & 0 & 0.0 & 0.0 & 2 & 0.3 & 162.8 & 1 & 1.1 & 1062.0 & 6 & 2.4 & 400.0 & 9 & 3.8 & 420.9 \\
\hline $40-49$ & 1 & .0 .1 & 148.1 & 20 & 0.9 & 44.0 & 4 & $0 . \dot{2}$ & 57.8 & 13 & 5.6 & 427.4 & 38 & 0.8 & 179.4 \\
\hline $50+\cdots$ & 0 & 0.0 & 0.0 & 32 & 1.4 & 42.4 & 6 & 0.3 & 54.0 & 10 & 3.3 & 334.7 & 48 & 5.0 & 104.8 \\
\hline TOTAL & 8 & 2.4 & 301.6 & 124 & 19.0 & 153.6 & 26 & 4.1 & $: 156.6$ & 58 & 26.8 & .461 .6 & 216 & 52.3 & 242.1 \\
\hline
\end{tabular}


TABLE IH-5

Comparison of 1977 Energy Consumption Between the Commercial And Government Office Buildings by Type of Heating Fuel

(Btu/Sq. Ft. in thousands; Square feet in millions)

Weighted Means

1977

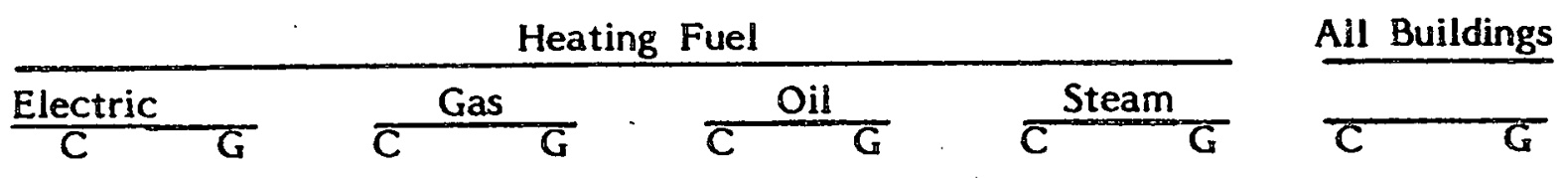

Electric Btu/Sq. Ft.

$$
114.978 .0
$$

92.4

54.3

75.591 .1

75.0

72.6

Gas Btu/Sq. Ft.

$86.0 \quad 48.7$

Oil Btu/Sq. Ft.

Steam Btu/Sq. Ft.

Total Btu/Sq.Ft.

Total Square Feet

Number of Buildings

$\begin{array}{rrrrrrrrrr}116.6 & 82.4 & 180.1 & 109.6 & 152.9 & 139.3 & 141.4 & 153.1 & 152.5 & 132.9 \\ 34.2 & 2.4 & 78.9 & 19 & 16.0 & 4.1 & 94 & 26.8 & 223.1 & 52.3 \\ 158 & 8 & 333 & 122 & 65 & 26 & 273 & 57 & 829 & 214\end{array}$

${ }^{1)}$ Commercial office building headings are denoted by " $\mathrm{C}$ ", and government office building headings are denoted by "G". 
TABLE III-6 ${ }^{1)}$

SUMMARY OF 1977 GOVERNMENT OFFICE BUILDING ENERGY USE (Btu/Sq. Ft. in thousands)

Census Division

and Type of Building. Fuel for Heating

Electricity $\quad \begin{gathered}\text { Number of } \\ \text { Buildings }\end{gathered}$

Other
Energy Use

Number of
Buildings

Buildings

Total
Energy Use

Number of Buildings

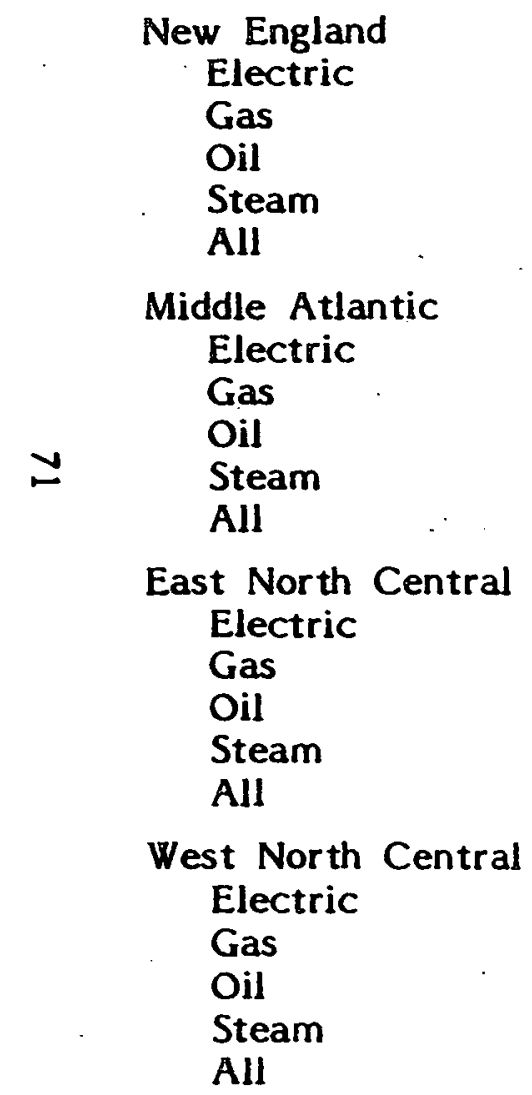

39.3

51.7

18.5

56.1

48.7

87.1

111.8

48.0

57.4

85.0

116.9

41.1

34.2

47.0

61.1

178.2

42.7

66.6

69.4

58.8
$78 . \overline{6}$

63.5

90.5

88.3

$26 . \overline{9}$

73.3

71.9

65.9

13

2
11

11
7
7

7
24

59.7

1
23

5

39
59.7

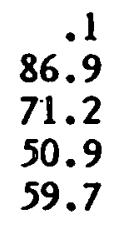

$60 . \overline{4}$

22.1

100.2

77.0
0
4
2
3
9

39.3

138.5

89.4

146.6

109.9

87.1

171.3

122.9

129.7

146.7

116.9

137.9

126.2

100.4

111.1

178.2

108.7

$99: 2$

170.2

134.7
1

4

3

10

1

3

13

2

11

7

24

23

23
5

10

39

1) See footnote at end of table. 
TABLE III-6, CONTINUED ${ }^{1)}$

SUMMARY OF 1977 GOVERNMENT OFFICE BUILDING ENERGY USE

(Btu/Sq. Ft. in thousands)

Census Division

and Type of Building Fuel for Heating

\section{Electricity}

Number of

Other
Energy Use

Number of Buildings

South Atlantic

Electric

Gas

Oil

Steam

All

164.7

East South Central Electric

N

Gas
Oil
Steam
All

West South Central

Electric

Gas

Oil

Steam

All

Mountain

Electric

Gas

Oil

Steam

All

1) See footnote at end of table.
31.8

80.2

84.1

0

8

29

42

$59 . \overline{8}$

49.2

$58 . \overline{1}$

$52 . \overline{0}$

-

52.0

18.0

48.4

60.3

68.8

49.8
$28 . \overline{1}$

27.5

80.5

75.4

$46 . \overline{7}$

37.5

$48 . \overline{8}$

39.6

.. -

40.3

48.1

68.2

11.2

44.0

\section{Total Energy Use}

$59 . \overline{9}$

203.3

161.0

159.5

110.7

87.5

$106 . \overline{8}$

11

0
18
0
0
18

$92 . \overline{3}$

-

92.3

18.0

97.0

128.5

80.0

91.7

$\begin{array}{rrr}30 & 97.0 \\ 2 & & 128.5 \\ 1 & & 80.0 \\ 34 & & 91.7\end{array}$

Number of Buildings 
TABLE III-6, CONTINUED ${ }^{1)}$

SUMMARY OF 1977 GOVERNMENT OFFICE BUILDING ENERGY USE (Btu/Sq. Ft. in thousands)

Census Division and Type of Building Fuel for Heating

Pacific

Electric

Gas:

Oil

Steam

All

National Average

Electric

W

Gas

Steam

All
37.2

36.5

52.8

56.1

43.5

78.0

54.3

91.1

72.6

67.6
Number of

Buildings

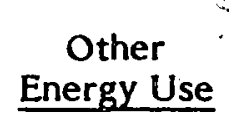

49.3

50.5

82.7

90.6

65.3

24.5

48.7

40.8

80.0

67.8

8
122
26
57
214

\section{Number of \\ Buildings}

25
57
208

Total

Energy Use

82.5

88.3

135.5

146.7

108.4

82.4

109.6

139.3

153.1

132.9
Number of

Buildings

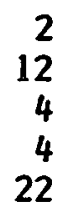

8

122

26

57

214

1) As is indicated in the text, "Electricity" plus "Other" may not exactly equal "Total". energy use. Similarly, the numbers of electric, gas, oil, and steam buildings do not exactly sum to equal the number of "All" buildings, for some buildings were not included in some computations. 
TABLE III - 7A

ENERGY CONSUMPTION IN THE 1977 BOMA OFFICE BUILDING SAMPLE (BTU/SO.FT. OOO)

HEIOHTED MEANS AND STANDARD DEVIATIONS

ELECTHICITY

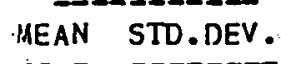

HEW ENGLAND

MI DULE ATLANTIC

EAST NORTH CENTHAL

WEST NIRTH CENTRAL

SIUTH ATLANTIC

EAST SIUTH CENTHAL

WEST SOUTH CENTRAL

MUUNTAIN

PACIFIC

NATIUNAL AVERAGE
48.7

11.4

85.0

38.9

61.1

3.7 .5

58.8

36.7

$84.1 \quad 47.9$

$58.1 \quad 26.8$

52.0

49.8

43.5

67.6

8.1

39.6

16.8

41.8

\section{OTHER FUELS \\ MEAN STD.DEV.}

88.3

$65.9 \quad 25.6$

$59.7 \quad 31.7$

$77.0 \quad 46.7$

$75.4 \quad 102.1$

$48.8 \quad 25.0$

$40.3 \quad 40.7$

44.0

48.7

65.3

58.6

67.8

71.6

$132.9 \quad 90.9$

TOIAL

MEAN STD.DEV.

$109.9 \quad 52.2$

$146.7 \quad 45.8$

$111.1 \quad 33.7$

$\begin{array}{ll}134.7 & 60.7\end{array}$

$\begin{array}{ll}159.5 & 126.9\end{array}$

$106.8 \quad 47.1$

$92.3 \quad 36.9$

$91.7 \quad 71.2$

108.456 .2

TABLE III - 78

ENERSY CONSUMPTION IN THE 1977 BOMA OFFICE BUILDING SAMPLE (BTU/SO.FT. ODO) UNWEIGHTED MEANS AND STANDARD DEVIATIONS

MEAN STD.DEV.

NEW ENGLAND

MIDOLE ATLANTIC

EAST NURTH CENTRAL

WEST NURTH CENTRAL

SOUTH ATLANTIC

EAST SIUUTH CENTRAL

WEST SOUTH CENTRAL

MOUNTAIN

PACIFIC

NATIUNAL AVERAGE
ELECTRICITY

UTHER FUELS

18.8

44.8

32.3

50.7

28.0

48.2

35.5

50.6

$65.8,45.4$

$47.5 \quad 26.0$

43.0

10.1

48.9

57.2

43.5

20.2

51.2

38.0

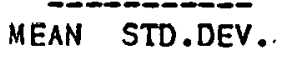

MEAN STD.DEV.

$86.7 \quad 38.7$

72.8

$41: 7$

76.8

43.4

79.4

51.7

59.9

69.7

39.9

20.5

64.1

49.2

69.8

46.5

54.6

51.9

67.6

52.3

\begin{tabular}{rr}
\multicolumn{2}{c}{ METAL } \\
\cline { 2 - 2 } 122.8 & 57.0 \\
\hline 117.9 & 48.0 \\
121.8 & 47.2 \\
128.0 & 58.6 \\
125.7 & 96.5 \\
87.5 & 41.8 \\
107.1 & 48.8 \\
116.8 & 72.5 \\
95.7 & 51.8 \\
117.0 & 66.6
\end{tabular}


TABLE III $-8 A$

ENERGY CONSUMPTION IN THE 1977 BOMA OFFICE BUILDING SAMPLE

ELECTRICALLY HEATED BUILDINGS

(BTU/SQ.FT. ODO)

WEI BHTED MEANS AND STANDARD DEVIATIUNS

GOVERNMENT OFFICE BUILDINGS

\begin{tabular}{|c|c|c|c|c|c|}
\hline \multicolumn{2}{|c|}{ ELECTRICITY } & \multicolumn{2}{|c|}{ OTHER } & \multicolumn{2}{|r|}{ TOTAL } \\
\hline MEAN & STD.DEV . & MEAN & STD.DEV . & MEAN & STD.DEV . \\
\hline$-\infty$ & $-\infty-\infty$ & -- & $-\infty-\infty$ & -- & - \\
\hline 39.3 & Unde f ined & 0.0 & 0.0 & 39.3 & Undef I ned \\
\hline 87.1 & Unde fined & 0.0 & 0.0 & 87.1 & Undefined \\
\hline 116.9 & 21.9 & 0.1 & Undefined & 116.9 & $1 \quad 21.9$ \\
\hline 178.2 & Undefined & 0.0 & 0.0 & 178.2 & Unde fined \\
\hline 0.0 & 0.0 & 0.0 & 0.0 & 0.0 & 0.0 \\
\hline 0.0 & 0.0 & 0.0 & .0 .0 & 0.0 & 0.0 \\
\hline 0.0 & 0.0 & 0.0 & 0.0 & 0.0 & 0.0 \\
\hline 18.0 & Undefined & 0.0 & 0.0 & 18.0 & Undefined \\
\hline 37.2 & 17.1 & 49.3 & Ündefined & 82.5 & 3.6 \\
\hline 78.0 & 47.6 & 24.5 & 24.6 & 82.4 & 45.3 \\
\hline
\end{tabular}

TABLE III - BB

ENERGY CONSUMPTION IN THE 1977 BUMA UFFICE HUILDING SAMPLF

ELECTRICALLY HEATED BUILDINGS

(BTU/SO.FT. ONO)

UNWEIGHTED MEANS AND STANDARD DEVIATIONS
GOVERNMENT OFFICE BUILOINGS

ELECTHICITY

MEAN STD.DEV.

$=-\infty$ -

NEW ENGLAND

MI DDLE ATLANTIC

39.3

0.0

87.1

0.0

.105 .2

35.1

178.2

0.0

0.0

0.0

0.0

0.0

0.0

0.0

18.0

0.0

63.2

44.0

82.4

53.6
OTHEH

\begin{tabular}{rr} 
VEAN & STD.DEV \\
\hline 0.0 & 0.0 \\
0.0 & 0.0 \\
0.1 & 0.0 \\
0.0 & 0.0 \\
0.0 & 0.0 \\
0.0 & 0.0 \\
0.0 & 0.0 \\
0.0 & 0.0 \\
49.3 & 0.0 \\
24.7 & 34.8
\end{tabular}

TOTAL

MEAN STO.DEV.

30.3

$87.1 \quad 0.0$

105.2 .35 .1

$178.2 \quad 0.0$

$0.0 . \quad 0.0$

$0.0 \quad 0.0$

$0.0 \quad 0.0$

$18.0 \quad 0.0$

$87.9 \quad 9.2$

$88.6 \quad 49.7$ 
TABLE III - 9 A

ENERGY CONSUMPTION IN THE 1977 BOMA OFFICE BUILDING SAMPLE GAS HEATED BUILDINGS

(BTU/SO.FT。0O0) WEIGHTED MEANS AND STANDARD DEVIATIUNS GOVERNMENT OFFICE BUILDINGS

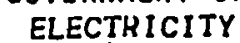

MEAN STD.DEV.

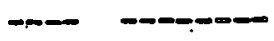

NEW ENGLAND

MIDULE ATLANTIC

EAST NOHTH CENTHAL

WEST NORTH CENTRAL

SOUTH ATLANTIC

EAST SOUTH CENTRAL

WEST SUUTH CENTRAL

MOUNTAIN

PACIFIC

NATIONAL AVERAGE

$51.7 \quad 11.4$

111.8

.41 .1

42.7

1.3 .7

$31.8 \quad 13.0$ !

$59.8 \quad 29.1$

52.0

48.4

36.5

54.3

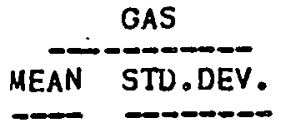

75.6

26.9

86.9

$60.4 \cdot 50.9$

28.126 .0

$46.7 \quad 26.0$

$39.6 \quad 39.4$

$48.1^{-} \quad 50.8$

50.5

48.7
28.8

19.8

37.9

39.2

43.8
TOTAL

MEAN STD.DEV.

$138.5 \quad 21.7$

$171.3 \quad 4.5 .9$

$137.9 \quad 42.0$

$108.7 \quad 52.5$

$59.9 \quad 27.9$

110.7 .50 .7

$92.3 \quad 36.9$

$97.0 \quad 76.9$

$88.3 \quad 40.3$

$109.6 \quad 59.6$

TABLE III - 9B.

ENERGY CONSUMPTIIN IN THE 1977 BOMA OJFFICE BUILDING SAMPLE GAS HEATED BUILDINGS. ;

(BTU/SO.FT. OOD) UNWEIGHTED MEANS AND STANDARD DEVIATIONS

GOVERNMENT OFFICE BUILDINGS

MI DDLE ATLANTIC

.EAST NORTH CENTRAL

WEST NORTH CENTRAL.

SOUTH ATLANTIC

EAST SOUTH CENTRAL

WEST SOUTH CENTRAL

MOUNTAIN

PACIFIC

NATIONAL AVERAGE
ELECTRICITY

MEAN STO.DEV.

56.1

45.5

17.5

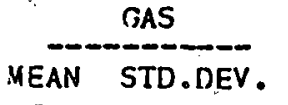

84.0

02.4

50.0

33.5

$44.7 \quad 20.5$

90.3

50.2

$44.1 \quad 26.6$

73.4

$: 57.3$

$37.7 \quad 18.9$

29.1

34.9

$47.3 \quad 27.4$

38.0

20.1

$43.0 \cdot 10.1$

62.6

47.8

72.9

47.4

38.8

32.8
14.2

36.0
63.2

48.2

\begin{tabular}{ll}
\multicolumn{1}{c}{ TOTAL } \\
MEAN & STD.DEV \\
\hline 158.4 & 51.6 \\
102.6 & 53.2 \\
140.1 & 55.4 \\
126.6 & 01.5 \\
66.8 & 34.6 \\
87.4 & 44.0 \\
107.1 & 48.8 \\
123.7 & 74.5 \\
35.9 & 33.3 \\
.112 .0 & 00.4
\end{tabular}


TABLE III - 10A

ENERGY CONSUMPTION IN THE 1977 BIIMA UFFICE BUILDINS SAMPLE OIL HEATED BUILDINGS

(BTU/SO.FT. OOO) WEIGHTED MEANS AND STANDARD DEVIATIUNS GOVERNMENT OFFICE BUILDINGS

ELECTRICITY

OIL

TOTAL

MEA'́N STD.DEV.

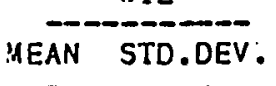

MEAN STD.DEV.

NE'N ENGLAND

18.5

3.8

63.5

38.7

89.4

4.9 .1

MIDDLE ATLANTIC

48.0

5.0

73.3

32.1

$122.9 \quad 26.9$

EAST NIRTH CENTRAL

$34.2 \quad 11.1$

71.2

$: 8.4$

126.2

32.9

WEST NORTH CENTRAL

$66.6 \quad 26.1$

22.1

13.9

99.2

21.8

SIUTH ATLANTIC

$164.7 \quad 59.2$

27.5

9.0

203.3

04.5 .

EAST SIUUTH CENTRAL

49.3 Undefined

37.5 Undefined

87.5 Undefined

WEST SUUTH CENTRAL

0.0

0.0

0.0

0.0

0.0

0.0

MOUNTAIN

60.3

8.7

68.2

8.0 .

128.5

16.7

PACIFIC

52.8

1.9 .0

82.7

32.6

135.5

51.1

NATIONAL AVERAGE

$91.1 \quad 63.5$

40.8

29.1

139.3

$63: 0$

TABLE III - 1OB

ENERGY CONSUMPTION. IN THE 1977 BOMA OFFICE BUILDING SAMPLE OIL HEATED BUILDINGS

(BTU/SO.FT. ODO) UNWEI GHTED MEANS AND STANDARD DEVIATIONS GOVERNMENT OFFICE BUILDINGS

ELECTRICITY

MEAN STD.DEV.

$-7$

18.7

5.3

43.2

9.1

39.6

15.6

48.8

32.3

71.2

68.8

49.3

0.0

0.0

0.0

51.4

17.5

44.3

19.4

48.3

34.8
OIL

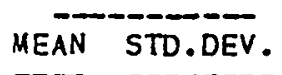

--D - -

65.6

54.8

97.3

66.9

8.3

24.3

18.3

0.0

37.5

0.0

0.0

60.2

16.0

62.8

39.3

$56.3 \quad 36.7$
TOTAL

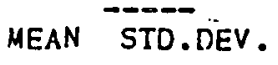

$91.9 \quad 69.5$

$.142 .8 \quad 5.6 .1$

$124.7 \quad 32.6$

$88.7 ; 19.7$

$103.8 \quad 75.1$

8.7. $5 \quad 0.0$

$0.0 \quad 0.0$

$111.6 \quad 33.5$

$107.6 \quad 57.2$

$108.3 \quad 47.8$ 
TABLE III - IIA

ENERGY CONSUMPTION IN THE 1977 BOMA OFFICE BUILDING SAMPLE STEAM HEATED BUILDINGS

(BTU/SO.FT. ODO) WEIGHTED MEANS AND STANDARD DEVIATIONS

GOVERNMENT OFFICE BUILDINGS

ELECTRICITY

STEAM

TOTAL

MEAN STD.DEV.

MEAN STD.DEV.

MEAN STD.DEV.

NEW ENGLAND

56.1

6.8

90.5

$21: 0$

146.6

.24 .6

MI DDLE ATLANTIC

57.4

1.7

.71 .9

30.6

$129.7 \quad 29.3$

EAST NORTH CENTRAL

47.0

25.0

50.9

13.4

$100.4 \quad 29.7$

WEST NORTH CENTRAL

69.4

39.7

100.2

32.6

170.2

57.2

SUUTH ATLANTIC

80.2

40.3

$80.5 \quad 106.9$

161.0

131.0

EAST SOUTH CENTRAL

0.0

0.0

0.0

0.0

0.0

0.0

WEST SIUTH CENTRAL

0.0

0.0

0.0

0.0

$0: 0$

0.0

MOUNTAIN

68.8 Unideftned

11.2 Undeftined

80.0

Undefined

PACIFIC

56.1

20.5

90.6

85.6

146.7

0.7 .5

NATIONAL AVERAGE

72.6

37.9

80.0

88.0

108.6

TABLE III - 11B

ENERGY CONSUMPTION IN THE 1977 BOMA OFFICE BUILDING SAMPLE STEAM HEATED BUILDINGS

(BTU/SO.FT。OOO)

UNWEIGHTEL MEANS AND STANRARQ DEV JATIONS

\section{ELECTRICITY}

MEAN STD.DEV.

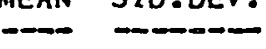

NEW ENGLAND

MIDDLE ATLANTIC

EAST NORTH CENTRAL

WEST NORTH CENTRAL

SOUTH ATLANTIC

EAST SOUTH CENTRAL

WEST SOUTH CENTRAL

M()UNTAIN

PACIFIC

NATIONAL AVERAGE
$.49 .1 \quad 11.7$

56.5

42.2

2.7

28.1

53.6

34.9

72.6

$44 \cdot 4$

0.0

0.0

0.0

0.0

68.8

0.0

54.2

22.2

62.1

$37: 9$
STEAM

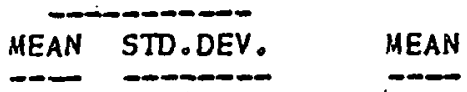

33.2

35.1

76.4

50.8

14.7

91.7

$41 \cdot 3$

72.8

.78 .2

0.0

0.0

0.0

0.0

11.3

0.0

92.9

95.7

74.0

64.4

123.

133.8

35.1

96.1

33.4

145.7

145.7

0.0

105.0

0.0

80.0

147.2

136.8

83.5 


\section{III-3 Energy Use Comparison: Downtown/Suburban}

Table III-12 compares energy use between downtown and suburban government office buildings on both a national and a regional basis. Most government buildings are located downtown, and the data are too limited to permit definitive conclusions. There is some evidence that suburban government gas and oil heated buildings use more energy than do downtown government buildings; this may be due to building construction practices. For example, suburban government building construction probably is less typically governmental in style and may not have the thermal retention properties of the governmental style. Only the weighted data are presented in Table III- 12 since the comparison appears to be of limited value. 
TABLE III - 12,1

DUMNTUWN ALD SUBURBAN BUILOINGS IN THE 1977 BOMA

OFFICE BUILDING SAMPLE

WEIGHTED MEANS AND STANUAKD DEVIATIONS

GOVERNMENT OFFICE BUILDINGS

NATIOHAL

TYPE OF HEATING FUEL

LICATION OF BUILDINGS

DUHHTHWN
TOTAL BTU/SO.FT. (000)

STANDARD DEVIATION 1000

SOUAKE FEET $(000,000)$

NURAER OF BUILDINGS

SO.FT./BLDG. (000)

SUBURBAN

TOTAL BTU/SO.FT. $(000)$

STANDARD DEVIATION 1000

SOUARE FEET $(000,000)$

NUMBER OF BUILDINGS

SO.FT./BLDG. $(000)$

$\begin{array}{rrrrr}73.5 & 107.0 & 95.5 & 153.5 & 133.1 \\ 39.9 & 55.6 & 25.4 & 110.1 & 94.8 \\ 1.6 & 13.8 & 2.1 & 26.1 & 43.5 \\ 6.0 & 111.0 & 20.0 & 57.0 & 194.0 \\ 262.5 & 124.1 & 103.7 & .457 .1 & 224.1\end{array}$

AVERASE ACROSS

$\begin{array}{llll}\text { ELECTRIC GAS WIL STEAM AL AL FUELS } & \end{array}$

$\begin{array}{rrrcr}98.9 & 113.5 & 184.9 & 89.2 & 126.3 \\ 50.1 & 70.1 & 57.8 & \text { Undefined } & 70.6 \\ 0.8 & 5.3 & 2.0 & 0.7 & 8.8 \\ 2.0 & 13.0 & 6.0 & 1.0 & 22.0 \\ 418.9 & 405.3 & 332.8 & 718.9 & 401.0\end{array}$


TABLE III - 12.2

DUWNTUWN AND SUBURBAN BUILDINGS IN THE 1977 BOMA

OFFICE BUILDING SAMPLE

WEIGHTED MEANS AND STANOARD DEVIATIONS

GOVERNMENT OFFICE BUILDINGS

NEW ENGLAND

TYPE OF HEATING FUEL.

LOCATION OF

DOWNTOWN

TUTAL BTU/SO.FT. (DOO)

STANDARD DEVIATION $10 D O$

SOUARE FEET $(000,000)$

NUMBER OF BUILDINGS

SO.FT。/BLDG。(000)

SUBURBAN

TUTAL BTU/SQ.FT. (OOO)

STANDARD DEVIATION 1000

SOUARE FEET $(000,000)$

NUMBER UF BUILDINGS

SO,FT。/BLDG。 $(000)$

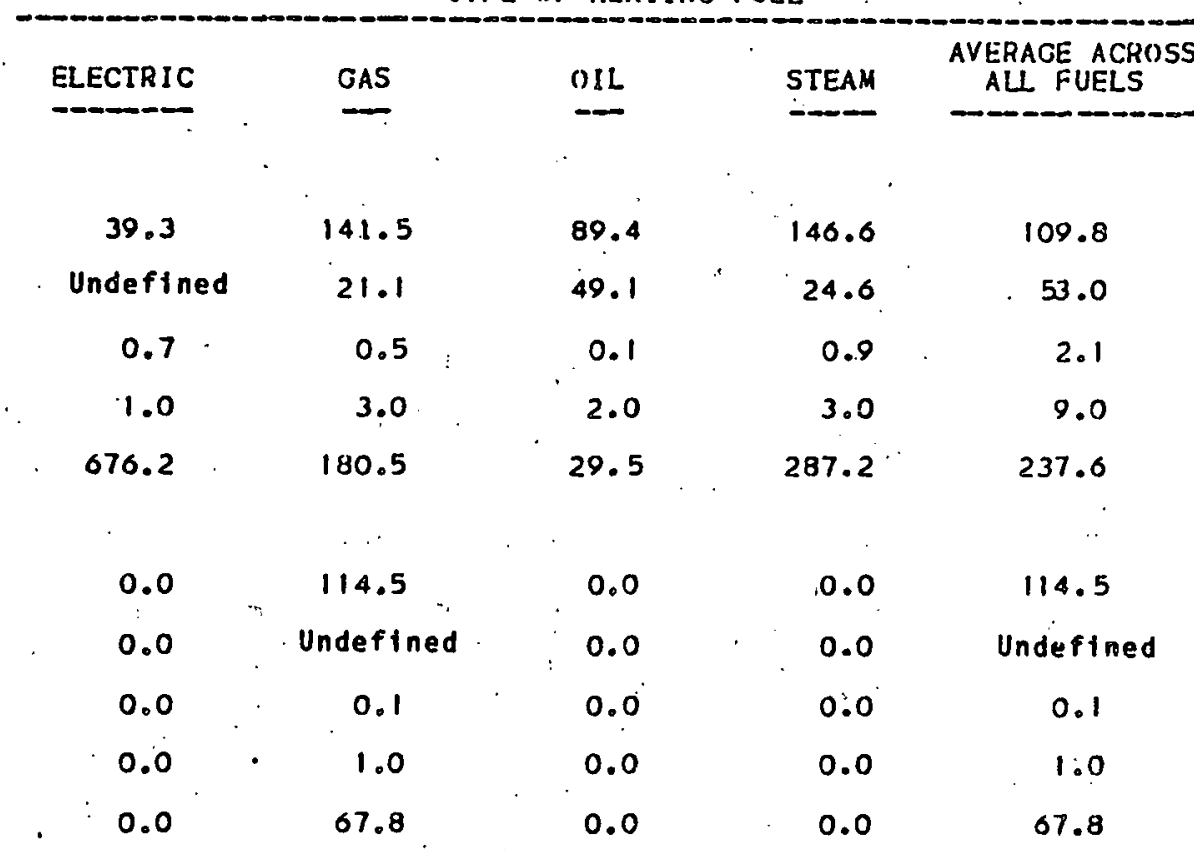

TABLE III- 12.3

DOWNTOWN AND SURURBAN BUIUUINTS IN THE 1977 BUMA OFFICE BUILDING SAMPLE WEIGHTED MEANS ANO STANDARD DEVIATIONS

GOVERNMENT OFFICE BUILIDINGS MIDDLE ATLANTIC

LOCATION OH BUILDINGS

DOWNTTOWN

TUTAL BTU/SO.FT. (000)

STANDARD DEVIATION 1000

SQUARE FEET $(000,000)$

NUMBER OF BUILDINGS

SO.FT./BLDG. $(000)$

SUBUKBAN

TOTAL BTU/SQ.FT. (000)

STANDARD DEVIATION $10 D O$

SOUARE FEET $(000,000)$

NUMBER OF BUILDINSS

SO.FI . /8LDG. . (000)

TYPE OF HEATING FUEL

\begin{tabular}{llll} 
ELECTRIC & GAS & SIL & STEAM \\
\hline
\end{tabular}

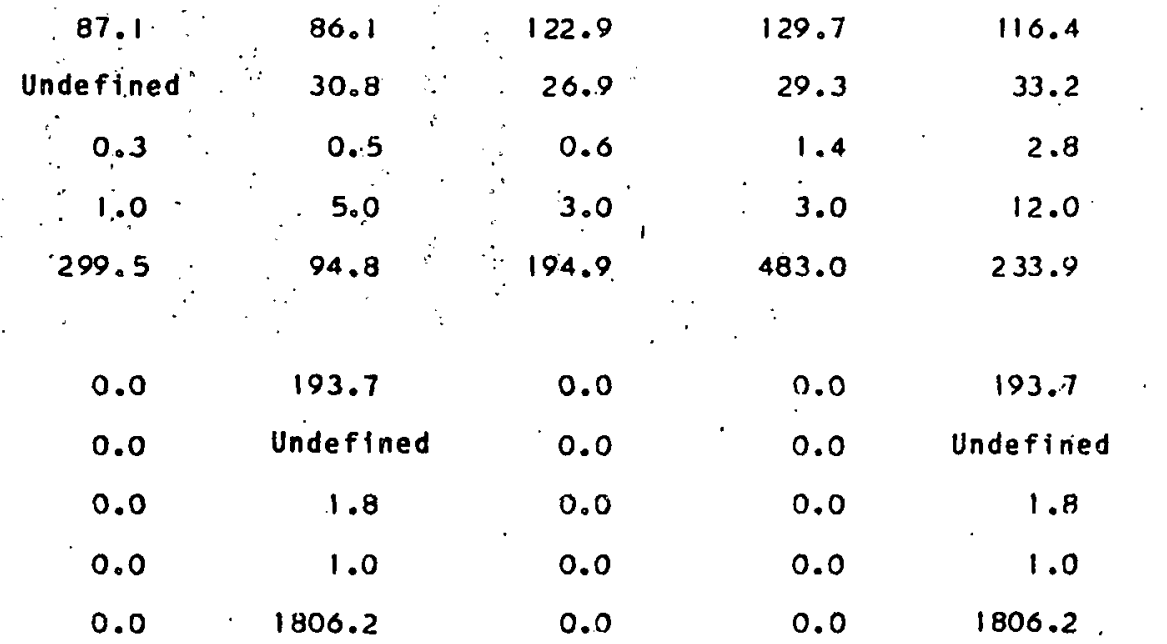


TABLE III - 12.4

DOWNTOWN AND SUBURBAN BUILDINGS IN THE 1977 BOMA OFFICE BUILDING SAMPLE

WEIGHTED MEANS AND STANDARD DEVIATIONS

GOVERNMENT OFFICE BUILOINGS

EAST NORTH CENTRAL

TYPE OF HEATING FUEL

LOCATION OF
BUILDINGS

\begin{abstract}
BUILDINGS
\end{abstract}
DOWNTOWN

TOTAL BTU/SO.FT. $(000)$

STANDARD DEVIATION $(000$

SOUARE FEET $(000,000)$

NUMBER OF BUILDINISS

SO.FT./BLDG. (000)

SUBURBAN

TUTAL BTU/SO.FT. (OOO)

STANDARD DEVIATION 1000

SQUAARE FEET $(000,000)$

NUMBER OF BUILDINTS

SO.FT ../BLDG. $(000)$

\begin{tabular}{|c|c|c|c|c|}
\hline 80.4 & 136.2 & 103.6 & .100 .4 & 105.9 \\
\hline Undefined & 40.5 & .18 .6 & 29.7 & 34.5 \\
\hline 0.2 & 0.6 . & 0.1 & 2.1 & 3.0 \\
\hline 1.0 & 10.0 & 3.0 & 7.0 & 21.0 \\
\hline 217.7 & $57 . \dot{5}$ & 29.4 & 305.4 & 143.8 \\
\hline 130.0 & 227.3 & 164.5 & 0.0 & 134.3 \\
\hline Undefined & Unde fined & Unidefined & 0.0 & 15.2 \\
\hline 0.6 & 0.0 & 0.1 & 0.0 & 0.7 \\
\hline 1.0 & 1.0 & 1.0 & 0.0 & 3.0 \\
\hline 605.4 & 11.0 & 52.0 & 0.0 & 222.8 \\
\hline
\end{tabular}

TÁBLE III - 12.5

DOWNTOWN AND SUBURBAN BUILOINGS IN THE 1977 BUMA

OFFICE BUILDING SAMPLE

WEIOHTEO MEANS AND STANDARD DEVIATIUNS

GOVERNMENT OFFICE BUILOINGS

WEST NURTH CENTRAL

TYPE OF HEATING FUEL

LOCATION OF

BU.ILDINOS

.DOWNTOWN

TUTAL BTU/SO,FT. (000)

STANDARD DEVIATION 1000

SDUARE FEET $(000,000)$

NUMBER OF BUILDINGS

Sn.FT. .BLDG . (000)

SUBURBAN

IUTAL BTU/SO.FT. (0OO)

STARIDARD DEVIATION 1000

SOUARE FEET $(000,000)$

NUMBER IF BUILDINGS

SO.FT./BLDG. (000)
ELECTRIC

OIL

STEAM

AVERAGE ACROSS

ALI FUELS

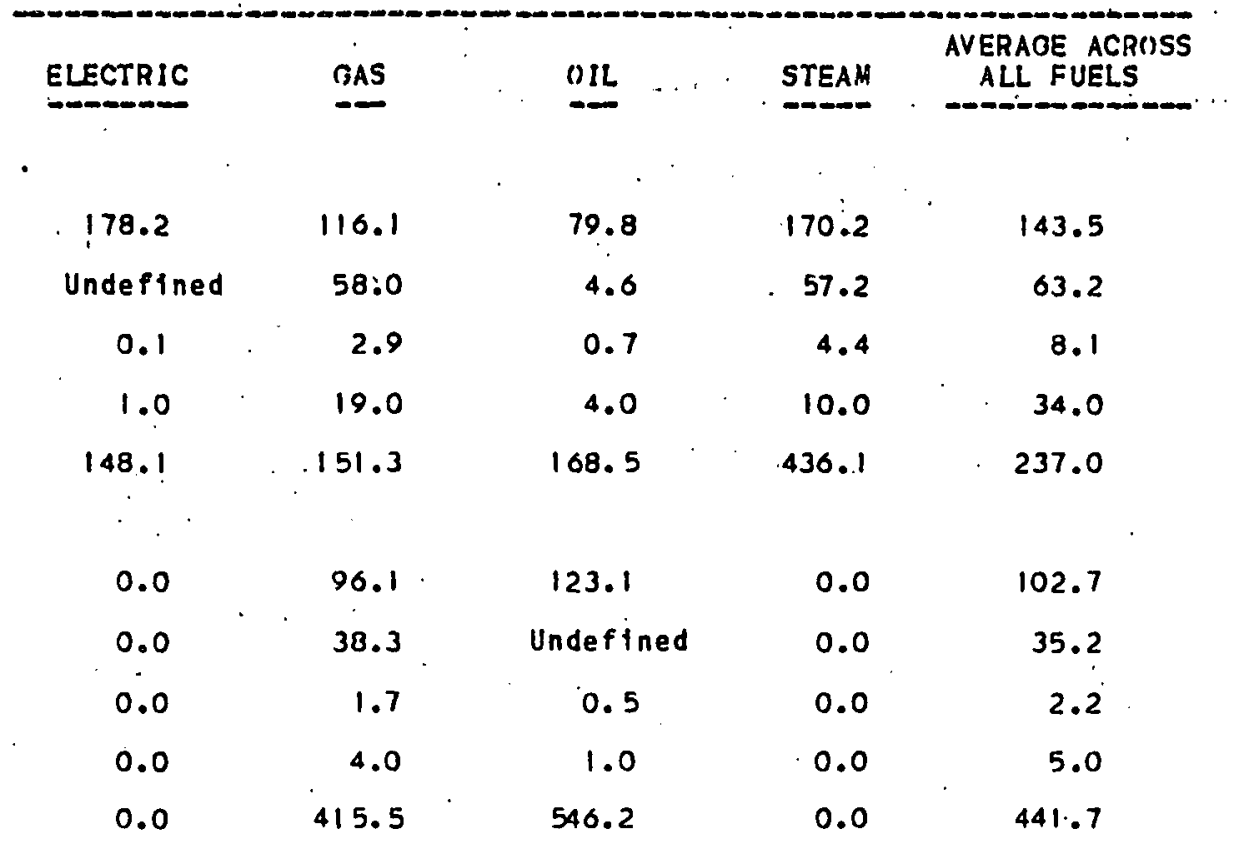


TABLE III - 12,6

DOWNTUWN AND SUBURBAN BUILDINOS IN THE 1977 BOMA

OFFICE BUILDING. SAMPLE

WEIGHTED MEANS AND STANDARD DEVIATIUNS

GOVERNMENT OFFICE BUILDINGS

SOUTH ATLANTIC

TYPE OF HEATING FUEL.

LUCATION OF

BUILDINGS

DUWNTUNN

TOTAL BTU/SO.FT. (000)

STANDARD DEVIATIUN 1000

SOUARE FEET $(000,000)$

NUMBER OF BUILDINIS

Sn.FT ./BLDG. (000)

SUBURBAN

TOTAL BTU/SO.FT. (000)

STANDARD DEVIATION 1000

SOUARE FEET $(000,000)$

NUMBER OF BUILDINGS

SO.FT./BLDG. (0OO)

ELECTRIC $\quad$ GAS $\quad$ OIL $\quad$ STEAM

AVERAGE ACROSS

ALL FUELS

$\begin{array}{rrrrr}0.0 & 59.9 & 82.3 & 164.3 & 158.4 \\ 0.0 & 27.9 & 16.7 & 133.1 & 131.5 \\ 0.0 & 0.8 & 0.1 & 15.5 & 16.5 \\ 0.0 & 8.0 & 3.0 & 28.0 & 39.0 \\ 0.0 & 101.6 & 48.8 & 554.3 & 422.6\end{array}$

$\begin{array}{rrrcr}0.0 & 0.0 & 218.5 & 89.2 & 169.1 \\ 0.0 & 0.0 & 50.8 & \text { Undefined } & 7.4 .4 \\ 0.0 & 0.0 & 1.2 & 0.7 & 1.9 \\ 0.0 & 0.0 & 2.0 & 1.0 & 3.0 \\ 0.0 & 0.0 & 582.5 & 718.9 & 627.9 .\end{array}$

TABLE III - 12.7

DUWNTUWN AND SUBURBAN BUILDINGS IN THE 1977 BOMA OFFICE BUILDING SAMPLE WEIGHTED MEANS AND STANDARD DEVIATIONS GOVERNMENT OFFICE BUILDINGS

EAST SOUTH CENTRAL

TYPE OF HEATING FUEL

LOCATION OF BUILDINGS

DOWNTOWN

T'ITAL BTU/SO.FT. $(000)$

STANDARD DEVIATION 1000

SOUARE FEET $(000,000)$

NUMBER OF BUILDINCS

SO.FT:/BLDG. (000)

SUBURBAN

TOTAL BTU/SO.FT. $(000)$

STANDARD DEVIATION 1000

SOUARE FEET $(000,000)$

NUMBER OF BUILDINTS

SO.FT. $/ B L D G .(000)$

$\begin{array}{rrcrr}0.0 & 110.7 & 87.5 & 0.0 & 106.8 \\ 0.0 & 50.7 & \text { Undeftned } & 0.0 & 47.1 \\ 0.0 & 1.7 & 0.3 & 0.0 & 2.1 \\ 0.0 & 10.0 & 1.0 & 0.0 & 11.0 \\ 0.0 & 173.9 & 347.6 & 0.0 & 189.7\end{array}$

0.0

0.0

0.0

0.0

0.0

0.0

0.0

0.0

0.0

0.0

0.0

0.0

0.0

0.0

0.0

0.0

0.0

0.0

0.0

0.0

0.0

0.0

0.0

0.0

0.0 
TABLE III - 12.8

DUWNTUWN AND SUBURBAN BUILDINGS IN THE 1977 BOMA

OFFICE BUILDING SAMPLE

WEIGHTED MEANS AND STANDARD DEVIATIONS

GOVERNMENT OFFICE BUILDINGS

WEST SOUTH CENTRAL

TYPE OF HEATING FUEL

LOCATION OFF
BUILDINGS

DOWNTUWN

TOTAL BTU/SO.FT. (OOO)

STANDARD DEVIATIUN 1000

SOUARE FEET $(000,000)$

NUMBER OF BUILDINGS

SO.FT ./BLDG . (000)

SUBURBAN

TOTAL BTU/SO.FT. $(000)$

STANDARD DEVIATION 1000

SOUARE FEET $(000,000)$

NUMBER OF BUILDINCS

SO.FT. /BLDG. (000)

ELECTRIC $\quad$ GAS $\quad$ UIL $\quad$ STEAM

AVERAGE ACROSS AL FUELS

$\begin{array}{rrrrr}0.0 & 92.3 & 0.0 & 0.0 & 92.3 \\ 0.0 & 36.9 & 0.0 & 0.0 & 36.9 \\ 0.0 & 2.7 & 0.0 & 0.0 & 2.7 \\ 0.0 & 18.0 & 0.0 & 0.0 & 10.0 \\ 0.0 & 149.9 & 0.0 & 0.0 & 149.9\end{array}$

0.0

0.0

0.0

0.0

0.0

0.0

0.0

0.0

0.0

0.0

0.0

0.0

0.0

0.0

0.0

0.0

0.0

0.0

0.0

0.0

0.0

0.0

0.0

0.0

0.0

TABLE III - 12.9

DUNNTUWN AND SUBURBAN BUILDINGS IN THE 1977 BOMA

OFFICE BUILDING SAMPLE

WEIGHTED MEANS AND STANDARD DEVIATIONS

GOVERNMENT OFFICE BUILDINGS

MOUNTAIN

TYPE OF HEATING. FUEL

\section{LOCATION OF BUILDINGS}

DONNTOWN

TOTAL BTU/SO.FT. $(000)$

STANDARD DEVIATION 1000

SOUAHE FEET $(0 \infty 0,000)$

NUMBER OF BUILDINGS

SO.FT . /BLDG. (000)

SUBURBAN

TOTAL BTU/SO.FT. $(000)$

STANDARD DEVIATION 1000

SOUARE FEET $(000,000)$

NUMBER OF BUILDINGS

SO.FT./BLDG. (000)

$\begin{array}{lr}0.0 & 138.2 \\ 0.0 & 7.3 .0 \\ 0.0 & 2.2 \\ 0.0 & 24.0 \\ 0.0 & 89.6\end{array}$

87.9

125.2

Undefined

Undefined

68.6

0.0

0.6

2.8

1.0

1.0

26.0

17.0

610.0

106.9

$\begin{array}{cr}18.0 & 45.4 \\ 0.1 & 43.3 \\ 0.2 & 1.7 \\ \text { Undefined } & 6.0 \\ 232.4 & 287.0\end{array}$

135.3
Undefined
0.1
1.0

0.0

46.7

0.0

45.3

0.0

2.1

0.0

8.0

100.9

0.0

256.9 
TABLE III - 12.10

DOWNTOWN AND SUBURBAN BUILOINGS IN THE 1977 BOMA

OFFICE BUILOING SAMPLE

WEIGHTED MEANS AND STANOAHD DEVIATIIUNS

GOVERNMENT OFFICE BUILOINGS

PACIFIC

TYPE IF HFATING FUEL

LOCATION OF

BUILDINGS

\begin{tabular}{|c|c|c|c|c|}
\hline ELECTHIC & GAS & OIL & STEAM & $\begin{array}{l}\text { AVERAGE ACRUSS } \\
\text { ALL. FUELS }\end{array}$ \\
\hline$-\cdots-$ & -- & - & --- & ------- \\
\hline
\end{tabular}

DONNTUWN

TOTAL BTU/SO.FT. $(000)$

STANDARD DEVIATION 1000

3.6

$-82.1$

89.3

116.5

93.7

SOUARE FEET $(000,000)$

0.2

44.9

11.2

84.5

61.0

NUMBER OF BUILDINOS

2.0

1.9

0.2

1.1

3.4

.116 .7

14.0

3.0

5.0

24.0

SUBURBAN

TOTAL BTU/SO.FT. (000)

STANDARD DEVIATION 1000

0.0
0.0
0.0
0.0
0.0

136.2

52.5

223.1

142.2

SOUARE FEET $(000,000)$

NUMBER OF BUILDINTS

SO.FT./BLDG. (000)

\begin{tabular}{|c|c|c|c|}
\hline 0.0 & 190.4 & 0.0 & 190.4 \\
\hline 0.0 & Unde fined & 0.0 & Undefined \\
\hline 0.0 & 0.1 & 0.0 & 0.1 \\
\hline 0.0 & 1.0 & 0.0 & 1.0 \\
\hline 0.0 & 132.8 & 0.0 & 132.8 \\
\hline
\end{tabular}




\section{III-4 Energy Consumption by Building Height}

Table III-13 presents energy use by building height. Most government buildings are less than 20 stories in height, and there does not appear to be any significant trend in energy use where only height is considered; energy use is lower for buildings in the 5-9 story category than for those in the 1-4 or 10-19 category. 
TABLE III-13A

ENEROY CONSUMPTION IN THE 1977 BOMA OFFICE BUILDINO SAMPLE

BY BUILOING HEIGHT

WEIOHTED MEANS ANI) STANDAKD DEVIATIONS

GOVERNMENT OFFICE BUILOINGS

\begin{tabular}{|c|c|c|c|c|c|c|}
\hline \multicolumn{7}{|c|}{ SIORIES } \\
\hline $1-4$ & $5-9$ & $10-19$ & $20-29$ & $30-39$ & $40-49$ & $50+$ \\
\hline
\end{tabular}

ELECTRIC BTU/SO.FT. (000)

MEAN

STAINDARO DEVIATION

UTHEH FUELS. BTU/SO.FT. (000)

SIEAN

STANDARD DEVIATION

TUTAL BTU/SO.FT. $(000)$

MEAN

STANDARD DEV IATIUN

TOTAL SOUARE FEET $(\infty 0,000)$

NUMBER OF BUILDINGS

$\begin{array}{lllllll}71.3 & 65.6 & : 07.4 & 86.7 & 59.0 & 56.1 & 0.0 \\ 63.3 & 37.4 & 39.3 & 38.1 & 13.9 & 0.2 & 0.0\end{array}$

62.6

$53.6 \cdot 39.1$

78.6

39.1

121.7

0.0

41.5

32.6

120.6

29.1

44.4

0.1

0.0

$\begin{array}{rrrrrrr}131.6 & 119.2 & 146.4 & 165.3 & 98.1 & 177.9 & 0.0 \\ 73.2 & 56.2 & 137.4 & 61.4 & 31.8 & \text { Undefined } & 0.0\end{array}$

$\begin{array}{llll}6.7 & 24.5 & 15.3 & 2.9\end{array}$

$2.9 \quad 0.9 \quad 1.6$

0.0

97.0

72.0

$37: 0$

4.0

3.0

1.0

0.0

ENEHTY CONSUMPTION IN THE 1977 BOMA OFFICE BUILDING SAMPLE

BY BUILDING HEIGLTT

UNIVIGHTED MEANS AND STANDARD DEVIATIOINS

GOVERNMENT OFFICE BUILDINGS

\begin{tabular}{|c|c|c|c|c|c|c|}
\hline \multicolumn{7}{|c|}{ STUHIES } \\
\hline $1-4$ & $5-9$ & $10-19$ & $20-29$ & $30-39$ & $40-49^{\circ}$ & $50+$ \\
\hline
\end{tabular}

ELECTHIC BTU/SO.FT. $(000)$

$\begin{array}{lllllllll}\text { MEAN } & 44.0 & 54.4 & 61.0 & 81.3 & 47.6 & 56.1 & 0.0 \\ \text { STANDARD DEVIATIIIN } & . & 41.2 & 32.0 & 39.2 & 41.1 & 18.8 & 0.0 & 0.0\end{array}$

OTHER FUELS BTU/SO.FT.(000)

MEAN

STANDARD DEVIATION

TOTAL BTU/SO.FT. $(000)$

STANIDARD DEVIATION

TOTAL SOUARE FEET $(000,000)$

NUMBER OF BUILUINGS
69.4

46.9

60.8

38.8

73.1

84.4

75.5

87.8

81.8

34.0

135.4

177.9

0.0

$112.0 \quad 115$.

61.5

56.2

93.9

67.8

65.0

0.0

0. 0

$\begin{array}{lll}6.7 & 24.5 & 15.3\end{array}$

97.0

72.0

37.0

2.9

0.8

1.6

0.0

4.0

3.0

1.0

0.0 
Table III-14 presents a comparison between commercial and government office buildings of energy consumption by building age. The decline in energy use is continuously downward through age 30 for government buildings. Energy use then rises above age 30, probably as a result of building renovation.

Table III-15 presents detailed data for government buildings. Trends in energy use probably can be explained in terms of equipment and usage. The newer buildings tend to have equipment that is more energy intensive than do the older buildings and are probably used to a greater degree. In addition, this newer equipment is probably in operation to a greater degree than older equipment, thereby causing energy consumption to be higher. In the 30-40 year age range, buildings that survive are typically remodeled; as is the case with the commercial sector this causes energy consumption to rise, for the buildings are then again used more intensively. 
TABLE III-14

Comparison of Energy Use by Age in Commercial and Government

Office Buildings

(Btu in thousands; square feet in millions)

Age

$\begin{array}{lllll}1-9 & 10-19 & 20-29 & 30-39 & 40-49\end{array}$

Electric Use-Btu/Sq. Ft. Commercial Buildings Government Buildings

$97.1 \quad 87$.

69.8

87.3

86.1

97.3

76.6

58.7

60.9

Other Fuels-Btu/Sq. Ft. Commercial Buildings

Government Buildings

64.2

92.4

45.8

58.3

81.9

69.8

45.2

Total Use-Btu/Sq. Ft.

Commercial Buildings

Government Buildings

146.1

61.6

74.1

62.4

73.0

87.3

37.5

65.9

$\infty$

Square Feet

Commercial Buildings

Government Buildings.

174.1

132.3

158.6

83.4

145.7

159.7

158.1

126.9

131.8

\section{1}

46.5

13.4

19.7

16.6

3.4

2.5
3.8

21.4

6.8

28.7

4.9

Number of Buildings

Commercial Buildings

Government Buildings

197

72

12

66

37

193 
TABLE III - 15A

ENEROY CONSUMPTION IN THE 1977 BOIMA UFFICE BUILDINO SAMPLE BY BUILDING AGE

DUWNTOWN BUILDINOS

WEIGHTED MEANS AND STANDARD DEVIATIONS

GOVERNMENT OFFICE BUILDINGS

$\begin{array}{ccccc}1-9 & 10-19 & 20-29 & 30-39 & 40-4.9 \\ \text { YEARS } & \text { YEAHS } & \text { YEAKS } & \text { YEARS } & \text { YE+ } \\ & \text { YEAHS } & \text { YEARS }\end{array}$

ELECTRIC BTU/SO.FT..(000)

MEAN

STANDARD DEVIATION
69.8

41.6
73.0

40.7

87.3

61.6

126.5
.45 .8

13.2

37.5

26.0

62.4

28.5

67.9

58.7

30.0

45.2

STANDARD DEVIATION

TUTAL BTU/SO.FT. (000)

MEAN
STANDARD DEVIATION

TOTAL SOUARE FEET $(000,000)$

NUMBER UF BUILDINGS

$\begin{array}{lrrrrr}149.7 & 132.3 & 83.4 & 159.7 & 126.9 & 111.1 \\ 147.2 & 62.0 & 28.0 & 70.9 & 42.4 & 47.5 \\ 13.4 & 19.7 & 3.4 & 3.8 & 0.8 & 4.9 \\ 48.0 & 61.0 & 12.0 & 9.0 & 37.0 & 47.0\end{array}$

TABLE III - 158

ENERGY CONSUMPTION IN THE 1.977 BOMAA OFFICE BUILDING SAMPLE BY BUILDING AGE DOWNTUWN BUILDINTS UNWEIGHTED MEANS AND STANDARD DEVIATIONS GOVERNMENT OFFICE BUILDINGS

$\begin{array}{cccccc}1-9 & 10-19 & 20-29 & 30-39 & 40-49 & 50+ \\ \text { YEARS } & \text { YEARS } & \text { YEARS } & \text { YEARS } & \text { YEAHS } & \text { YEARS }\end{array}$

ELECTRIC BTU/SO.FT. (OOO)

MEAN

STANDAKD DEVIATION

OTHER FUELS BTU/SO.FT. 1000$)$

MEAN

STANDAKD DEVIATION

TUTAL BTU/SO.FT. (OOO)

MEAN
STANDARD DEVIATION

TOTAL SNUARE FEET $(000,000)$

NUMBER OF BUILDINGS

58.9

28.3

76.9

58.9

39.7

131.0

90.9

122.4

68.3

93.6

141.3

46.3

71.8

3.4

3.8

12.0

48.0

61.0

9.0

37.0

109.0

103.2

42.6

49.0

13.4

19.7

6.8

4.9 


\section{III-6 Energy Consumption and Computer Space}

Tables IIl-16 and III-17 present energy consumption for government buildings with and without computer space. As was the case with commercial office buildings, government buildings with computer space tended to use more electricity and less "other" fuels. It is possible that the increased energy use for electricity could be directly due to the installed machinery. However, another possible explanation would be that the increased building usage associated with a computer installation was responsible for the higher electric use and that the waste heat from increased levels of lighting and other building equipment resulted in a decreased need for building heating. This latter explanation appears to be more likely given the heat transfer characteristics of buildings. The recapture of waste heat from a specific source such as a computer room is probably relatively rare and, accordingly, it is likely that waste heat from computer operations is exhausted in many cases to the outside of the building rather than to other locations within the building for heating purposes. However, it is likely that a number of heat generating sources in the building in addition to the computer space are in operation to a greater than normal degree in order to accommodate the personnel involved with the computer operations. Therefore, the heat associated with lighting and office equipment in the other areas may also contribute to the reduced heating needs. 


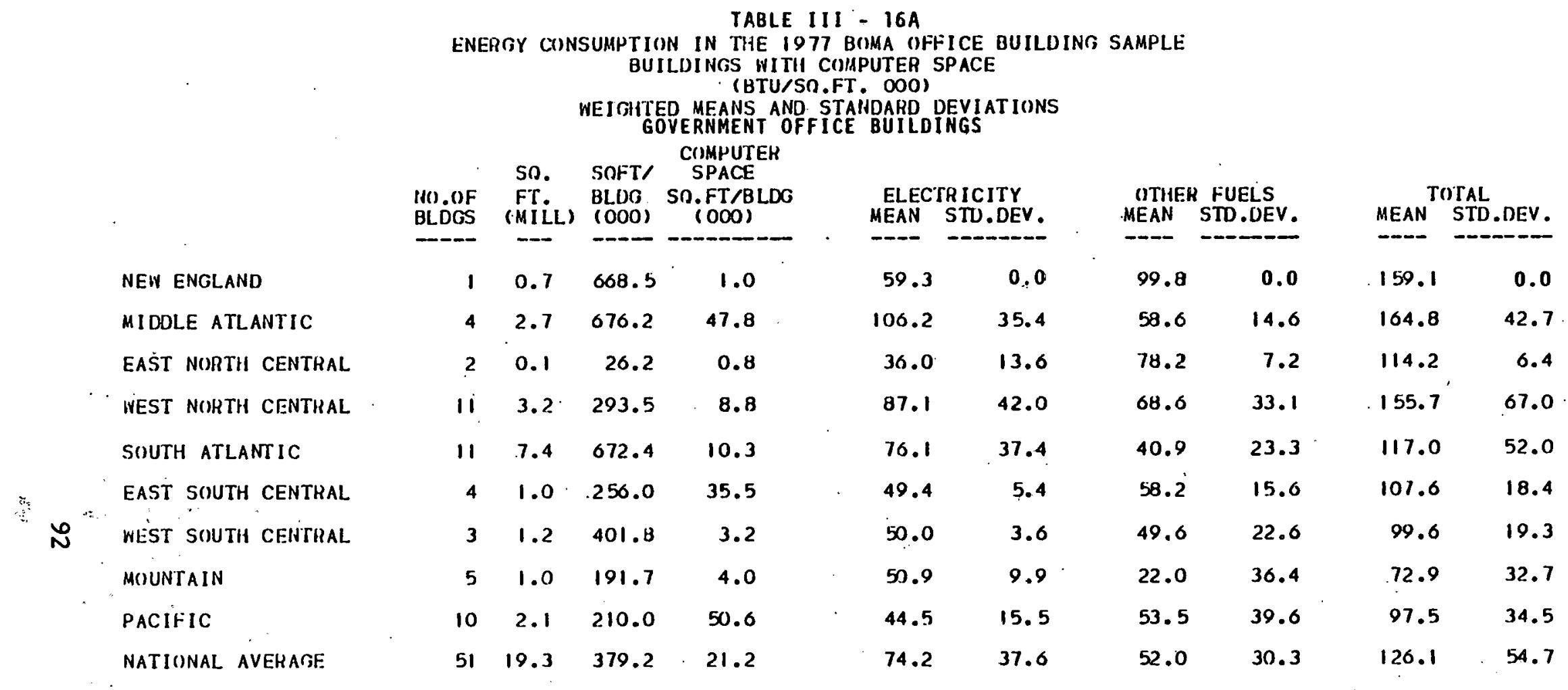


TABLE III - 168

ENERGY CONSUMPTION IN THE 1977 BOMA OFFICE BUILDING SAMPLE

BUILUINGS WITH COMPUTER SPACE

UNHEIGHTED MEANS AND STANDARD DEVIATIONS

COMPUTER

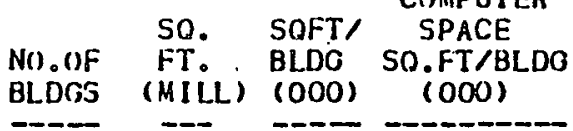

NEW ENGLAND

- hidDLE aTLANTIC

EAST NURIH CENTRAL

WEST NOKTH CENTHAL

SUITH ATLANTIC.

EAST SUUTH CENTRAL

WEST SOUTH CENTKAL

HOUNTAIN

PACIFIC

NATIONAL AVERAGE $\begin{array}{llll}4 & 2.7 & 676.2 & 47.8\end{array}$

$\begin{array}{llll}2 & 0.1 & 26.2 & 0.8\end{array}$

$\begin{array}{llll}11 & 3.2 & 293.5 & 8.8\end{array}$

$\begin{array}{llll}11 & 7.4 & 672.4 & 10.3\end{array}$

$4 \quad 1.0 \quad .256 .0 \quad 35.5$

$\begin{array}{llll}3 & 1.2 & 401.8 & 3.2\end{array}$

$\begin{array}{llll}5 & 1.0 & 191.7 & 4.0\end{array}$

$\begin{array}{llll}10 & 2.1 \quad 210.0 \quad 50.6\end{array}$

$\begin{array}{llll}51 & 19.3 \quad 379.2 & 21.2\end{array}$ $\begin{array}{llll}1 & 0.7 & 668.5 & 1.0\end{array}$
ELECTRICITY MEAN STD.DEV.

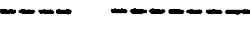

$59.3 \quad 0.0$

$72.5 \quad 39.4$

$32.6 \quad 19.9$

$68.3^{\circ} 37.2$

$71.3 \cdot 37.9$

36.7

16.5

42.1

46.2

$$
8.0
$$

21.0

48.1

22.6

57.9

31.9
OTHER FUELS MEAN STD.DEV.

\section{--D - - - -}

99.1

0.0

63.2 .25 .1

$80.0 \quad 10.4$

$61.8 \quad 31.2$

48.3

28.7

16.7

.45 .3

71.2

46.7

49.4

46.1

53.1

40.3

32.3

. 57.0

.159 .1

135.7

$112.5 \quad 9.4$

$130.0 \quad 55.7$

$119.6 \quad 60.2$

$82.1 \quad 30.1$

$113.3 \quad 44.3$

$95.7 \quad 43.3$

94.534 .1

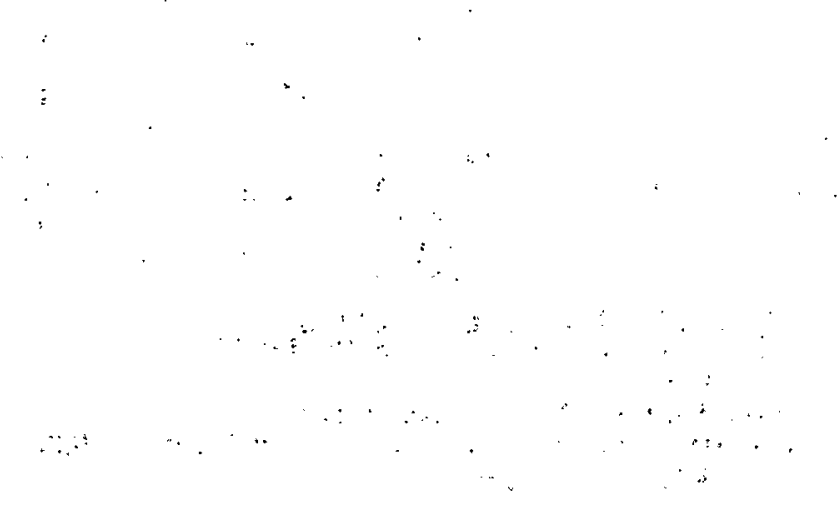


TABLE -111 - 17A

ENEHGY CONSUMPTION IN ITHE 1977 BOMA OHFICE BUILDING SAMPLE BUILDINCS WITHUUT COMPUTER SPACE

(BTU/SD.FT. ODO)

WEIGHTED MEANS AND STANDARD DEVIATIUNS

GTED MEANS AAN STANDARD DEVIAT
GOVERNMENT OFFICE BUILOINGS

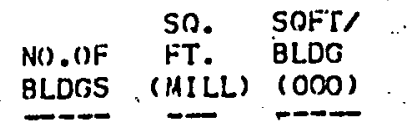

ELECTRICITY

MEAT STD. DEV.

OTHER FUELS

MEAN STD.DEV.

NEW ENGLAND

$\begin{array}{lll}9 & 1.5 & 170.9\end{array}$

44.1

10.9

79.3

22.7

MIDDLE ATLANTIC

$9 \quad 1.9212 .1$

55.0

18.6

78.1

34.1

EAST NIRTH CENTRAL

WFST NUHTH CENTHAL

SJUTH ATLANTIC

EAST SIUUTH CENTKAL

WEST SOUTH CENTRAL

MOUNTAIN

PACIFIC

NATIONAL AVERAGE

NEN ENGLAND

MIDDLE ATLANTIC

EAST NORTH CENTHAL

WEST NORTH CENTRAL

SOUTH ATLANTIC

FAST SOUTH CENTRAL

WFST SUUTH CENTKAL

MUUNTAIN

PACIFIC

NATIONAL AVERAGE

22

22

28

3

11.0353 .8

$\begin{array}{lll}7 & 1.1 & .151 .7\end{array}$

61.5

37.6

45.9

24.9

89.4

53.1

66.4

35.3

$\begin{array}{lll}15 & 1.5 & 94.6\end{array}$

53.6

10.1

49.5

43.9

42.2

18.2

63.9

43.6
96.4

166

$$
33.0 \quad 198.7
$$$$
43.6
$$

TABLE III - 178

ENERGY CONSUMPTION IN THE 1977 BOMA OFFICE BUILDING SAMPLE BUILOINGS WITHOUT COMPUTEH SPACE (BTU/SO.FT. OOO) UNIVEIGHTED MEANS AND STANDARD DEVIATIUNS

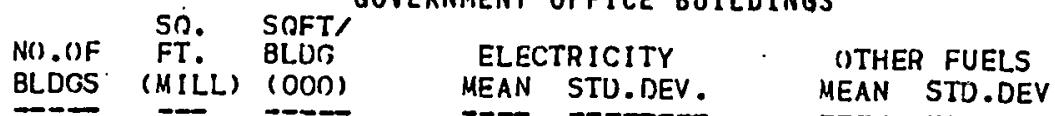
GOVERNMENT OFFICE BUILDINGS

$$
\text { BLDCS }
$$

$9 \quad 1.9212 .1$

$22 \quad 3.6 \quad 165.2$

$\begin{array}{lll}28 & 7.0 & 251.3\end{array}$

$31 \quad 11.0 \quad 353.8$

$7 \cdot 1.1 \quad 151.7$

$\begin{array}{lll}15 & 1.5 & 99.6\end{array}$

$30 \quad 3.9 \cdot 129.6$

$15 \quad 1.4 \cdot 96.4$

--D -

$43.2 \quad 19.2$

$41.0 \quad 25.3$

49.0 .28 .6

$43.6 \cdot 33.0$

$63.8,48.2$

53.7

29.6

43.2

10.7

49.4

01.5

41.0

19.2

49.3

39.5

166
MEAN STD.DEV.

\section{$85.0 \quad 41.0$}

$77.5 \quad 48.8$

76.5

45.5

86.6

57.0

$64.0 \quad 79.3$

36.9

23.1

62.6

51.2

73.3

46.4

55.4

58.2

70.8

56.7
TOTAL

MEAN STU.DE $\psi$.

$88.6^{\cdots} \quad 49.0$

$120.9 \quad 36.6$

111.0

125.1

188.1 .152 .0

$100.1 \quad 63.4$

86.5

90.4

123.3

130.8

TUTAL

MEAN STU.DEV.

$110.8 \quad 58.9$

$109.9 \quad 49.4$

$122.6 \quad 49.3$

$127.2 \quad 60.7^{10}$

$127.9 \quad 107.2$

$90.5 \quad 49.2$

$105.8 \quad 51.0$

$120.3 \quad 76: 3$

90.4

117.9 


\section{III-7 Energy Consumption and Building Air Conditioning Equipment}

Building energy consumption is presented in terms of air conditioning equipment in Tables III-18, III-19, and III-20. Electric air conditioning was present in 164 buildings (Table III-18); gas air conditioning was present in 4 buildings (Table 19); steam absorption was present in 17 buildings (Table 20); and two buildings had steam turbine air conditioning. Buildings with electric air conditioning used more electricity but less fuel than buildings with steam absorption. 
TABLE III - 18A

ENERGY CONSUMPTION IN THE 1977 BOMA OFFICE BUILDINF SAMPLE BUILDINGS WITH ELECTRIC AIR CONDITIONING (BTU/SA.FT. OOO)

WEIGHTED MEANS AND. STANDARD DEVIATIUNS

GOVERNMENT OFF.ICE BUILDINGS

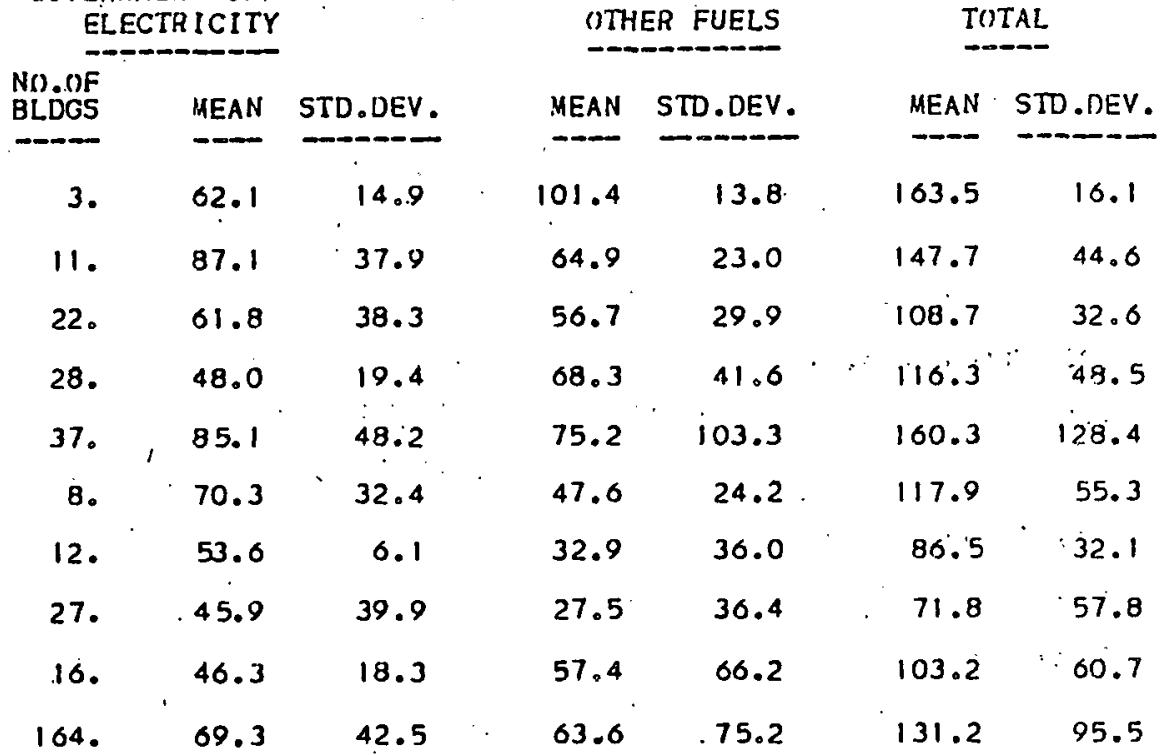

TABLE III - 18B

ENERGY CONSUAPTION IN THE $1977^{\circ}$ BOMA OFFICE BUILDING SAMPLE BUILDINGS WITH ELECTRIC AIR CONDITIONING (BTU/SO.FT. OOO) UNIYEIGHTED MEANS AND STANDARU DEVIATIOHS , GOVERNMENT OFFICE BUILDINGS

\begin{tabular}{|c|c|c|c|c|c|c|c|}
\hline & TRICITY & th & $\therefore$ & OTH & ER FUELS & & IAL \\
\hline $\begin{array}{l}\mathrm{N}() .0 F \\
\text { BLDGS }\end{array}$ & MEAN & STO.DEV. & & AEEAN & STD.DEV.' & MEANH & STO.DE \\
\hline & $-\infty$ & 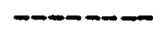 & & $-\infty$ & $---\infty-$ & $-\infty$ & 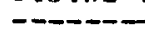 \\
\hline 3. & 54.7 & 23.1 & . & 123.2 & 29.7 & 177.9 & 45.9 \\
\hline 11. & 55.1 & 33.3 & & 64.9 & 29.3 & 114.1 & 41.6 \\
\hline 2.2 & 48.2 & 29.3 & & 7.5 .1 & $44: 6$ & 119.9 & 48.5 \\
\hline 28. & 42.5 & 22.2 & & 74.5 & 50.8 & 117.0 & .56 .4 \\
\hline 37. & .67 .9 & 47.7 & & 60.6 & 7.2 .2 & 128.5 & 101.5 \\
\hline 8. & 47.7 & 30.3 & ' & 40.9 & 18.9 & 88.6 & 45.8 \\
\hline 12. & 43.7 & 9.1 & & 57.1 & 51.9 & 100.7 & 50.1 \\
\hline 27. & 50.9 & 63.9 & & 60.8 & 46.4 & 109.5 & 76.7 \\
\hline 10 & 44.5 & 22.0 & & 48.8 & 54.3 & 90.2 & 50.6 \\
\hline 164. & 52.0 & 40.0 & & 64.1 & 53.8 & 114.5 & 70.0 \\
\hline
\end{tabular}


TABLE III - 19A

ENERGY CONSUMPTION IN THE 1977 BOMA OFFICE BUILDING SAMPLE

BUILDINGS WITH GAS AIR CONDITIUNING

(BTU/SO.FT. OOO)

WEIGHTED HEANS AND STANDAHD DEVI ITIONS

GOVERNMENT OFFICE BUILDINGS

ELECTR ICITY

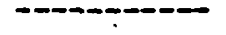

MEAN STD.DEV.

$0.0 \quad 0.0$

$0.0 \quad 0.0$

$0.0 \quad 0.0$

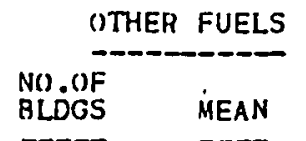

STD.DEV.

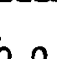

0.

0.0

0.0

0.0

0.0

27.9 Undefined

33.2 Undefined

0.0

0.0

0.0

0.0

81.9

30.1

0.0

0.0

55.4

34.2
0.0

0.0

o.

0.0

l. 182.0

Undefined

1.

111.6

Undefined

0.

0.0

0.0

0.0

0.0

2. 115.9

27.2

0.0

0.0

0.

4. $144.8 \quad 38.3$

4. $144.8 \quad 38.3$
TUTAL

MEAN STD.DEV.

$0.0 \cdot 0.0$

$0.0 \quad 0.0$

$0.0 \quad 0.0$

209.9 Undefined

144.7 Undefined

$0.0 \quad 0.0$

$0.0 \quad 0.0$

$197.8 \quad 57.3$

$0.0 \quad 0.0$

$200.2 \quad 43.2$

TABLE III - 19B

ENERGY CONSUMPIION IN THE 1977 BOMA OFFICE HUILDING SAMPLE BUILDINGS WITH GAS AIR CONUITIONING (BTU/SO.FT. OOO) UNIVEIGHTED MEANS AND STANDARD DEVIATIONS GOVERNMENT OFFICE BUILDINGS

\section{ELECTH ICITY}

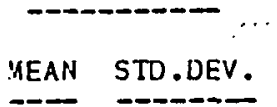

NEW ENGLAND

MIDDLE ATLANTIC

EAST NOATH CENTRAL

WEST NORTH CENTRAL

SOUTH ATLANTIC

EAST SIUTHH CENTRAL

WEST SOUTH CENTRAL

MoUUTAIN

PACIFIC

NATIONAL AVERAGE

$\begin{array}{rr}0.0 & 0.0 \\ 0.0 & 0.0 \\ 0.0 & 0.0 \\ 27.9 & 0.0 \\ 33.2 & 0.0 \\ 0.0 & 0.0 \\ 0.0 & 0.0 \\ 66.7 & 47.6 \\ 0.0 & 0.0 \\ 48.6 & 34.6\end{array}$

TOTAL

MEAN STD.DEV.

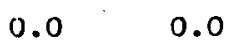

0.

0.0

0.0

0.0

0.0

0 .

0.0

0.0

0.0

0.0

1. $\quad 182.0$

0.0

209.9

0.0

1. $\quad 111.6$

0.0

144.7

0.0

0.

0.0

0.0

0.0

0. $\quad 0.0$

0.0

0.0

0.0

2. 102.1

43.1

168.8

90.7

0.

0.0

0.0

0.0

0.0

4. 124.5

45.9

173.1 .59 .0 
TABLE [I]-20A

ENEROY CONSUMPTIIN IN THE 1977 BIIMA OFFICE AUILOINO SAMPLE

BUILDINOS WITH STEAM ABSURPTION ALR CINDITIININO

(BTU/SO.FT. ODO)

WEIOHTED MEANS AND STANDAHD DEVIATIUNS

GOVERNAENT OFFICE BUILOINGS

\begin{tabular}{|c|c|c|c|c|c|c|c|c|}
\hline \multirow[t]{2}{*}{$\cdot$} & \multirow{2}{*}{. } & \multicolumn{4}{|c|}{ ELECTRICITY OTHER FUELS } & \multirow[b]{2}{*}{ STD.DEV. } & \multicolumn{2}{|c|}{ TOTAL } \\
\hline & & MEAN & STD.DEV. & $\begin{array}{l}\text { No.UF } \\
\text { BL.DOS }\end{array}$ & MEAN & & MEAN & STD.DEV. \\
\hline NEW ENOLAND & & 58.2 & 2.7 & 2. & .90 .8 & 22.0 & 1.49 .0 & 24.7 \\
\hline MIDDLE ATLANTIC & & 0.0 & 0.0 & 0 . & 0.0 & 0.0 & 0.0 & 0.0 \\
\hline EAST' NORTH CENTRAL & & 48.5 & 0.9 & 2. & 108.7 & 17.1 & 157.3 & 18.0 \\
\hline WEST NORTH CENTRAL & & 61.1 & 34.5 & 6. & 86.0 & 21.9 & 147.1 & 29.8 \\
\hline SUUTH ATLANTIC & & 0.0 & 0.0 & 0. & 0.0 & 0.0 & 0.0 & 0.0 \\
\hline EAST SOUTH CENTRAL & & 0.0 & 0.0 & 0. & 0.0 & 0.0 & 0.0 & 0.0 \\
\hline WEST SOUTH CENTRAL & & 49.8 & 12.4 & 2. & 123.1 & 2.3 & 173.0 & 10.2 \\
\hline MUUUNTÁIN & & 38.0 & Undefined & i. & 115.2 & 0.0 & 153.2 & 0,0 \\
\hline PACIFIC & & 34.5 & 3.1 & 4: & 74.3 & $39.5^{\circ}$ & 108.8 & 40.7 \\
\hline NATIUNAL AVERAGE & & 49.1 & 21.2 & 17. & 87.7 & 31.3 & 136.9 & 37.5 \\
\hline
\end{tabular}

TABLE III-20B

ENEROY CONSUMPTION IN THE 1977 BOMA OFFICE BUILDING SAMPLE BUILDINGS WITH STEAM ABSORPTION AIR CONDITIONING

(BTU/SO.FT, ODO) UNWEITHITED MEANS AND STANDARD DEVIATIUNS GOVERNAENT OFFI CE BUILDINGS

$$
\text { ELECTRICITY }
$$

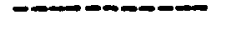

MEAN STD.DEV.

$55.5 \quad 5.3$

NEW ENGLANO

MI DDLE ATLANTIC

$0.0 \quad 0.0$

EAST NORTH CENTRAL

$47.8 \quad 1.6$

WEST NORTH CENTRAL

SOUTH ATLANTIC

EAST SOUTH CENTRAL

NEST SOUTH CENTRAL

MOUNTAIN

PACIFIC

NATIOUNAL AVERAGE

$53.6 \quad 37.0$

$.0 .0 \quad 0.0$

$0.0 \quad 0.0$ :

$51.4 \quad 17.7$

$38.0 \quad 0.0$

34.6

3.5

$4.7 .5 \quad 22.9$
OTHÉR FUELS

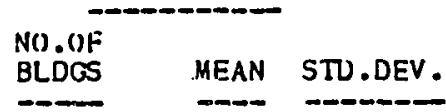

2.

68.5

44.4

0.0

0.0

123.9

49.7

0 .

94.7

31.3

0.0

0.0

6. 98.9

39.6

142.5

32.9

0.

0.0

0.0

1.52 .6

42.4

o.

0.0

0.0

0.0

0.0

- 2.

122.8 .

3.1

0.0

0.0

0.0

.174 .2

14. 5

4. 68.5

46.6

153.2

0.0

i7. 91.5

38.2 .

103.0

48.0

138.9

42.7 . 
TABLE III, $21 \mathrm{~A}$

ENEROY CONSUMPTION IN THE 1.977 BOMA OFFICE OUILDINM SAMPLE

BUILDINOS HITH STEAM TURBINE AIR CONDITIUNINO

(BTU/SO.PT. 0OO)

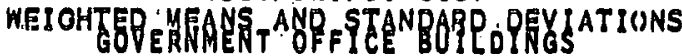

ELECTRICITY

MEAN STD.DEV. $\quad$ NULDOF MEAN STD.DEV. MEAN STD.DEV.

NEW ENSLLAND

0.0

0.0

0.

0.0

0.0

0.0

0.0

MIDOLE ATLANTIC

0.0

0.0

0.

0.0

0.0

0.0

0.0

EAST NURTH CENTHAL

$0.0 \quad 0.0$

0 .

0.0

0.0

0.0

0.0

WEST NORTH CENTRAL

142.9 Undefined

1. 107.7

0.0

250.6

0.0

SOUTH ATLANTIC

0.0

0.0

$0.0^{\circ} \quad 0.0$

0.0

0.0

0.0

EAST SOUTH CENTRAL

51.0

0.0

1. 70.2

0.0

121.2

0.0

WEST SUUTH CENTRAL

0.0

0 .

0.0

0.0

0.0

0.0

MOUNTAIN

0.0

0.0

o.

0.0

0.0

0.0

0.0

PACIFIC

0.0

0.0

0.

0.0

0.0

0.0

0.0

NATIONAL AVERAGE

$105.1 \quad .45 .2$

2.

92.3

18.4

197.4

63.7

TABLE III - 218

ENERGY CONSUMPTION IN THE 1977 BUMA OFFICE BUILDING SAMPLE BUILDINGS WITH STEAM TURBINE AIR CONDITIONING

(BTU/SO.FT. OOO) UNWEICHITED MEANS AND STANDARD DEVIATIONS GOVERNMENT OFFICE BUILDINGS

ELECTR I CITY

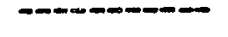

MEAN STD.DEV。

$$
0.0
$$

0.0

$0: 0$

0.0

0.0

0.0

142.9

0.0

0.0

0.0

51.0

0.0

EAST SOUTH CENTRAL

0.0

0.0

0.0

0.0

0.0

0.0

96.9

65.0
OTHER FUELS

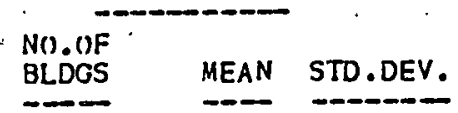

0.

0.0

0.0

0 .

0.0

0.0

0.0 .0

0.0

1. 107.7

0.0

o. 0.0

0.0

1. 70.2

0.0

0.

0.0

0.0

0 .

0.0

0.0

0.

0.0

0.0

2. $89.0 \quad 26.5$

2. $89.0 \quad 26.5$
TOTAL

MEAN STD.DEV.

$0.0 \quad 0.0$

0.010 .0

$0.0 \quad 0.0$

$250.6 \quad 0.0$

$0.0 \quad 0.0$

$121.2 \quad 0.0$

$0.0 \quad 0.0$

$0.0 \quad 0.0$

$0.0 \quad 0.0$

$185.9 \quad 91.5$ 


\section{III-8 Conclusions on Energy Use in Government Office Buildings}

The 1977 data base for government of fice buildings was not representative of government buildings in general either in terms of location or age of buildings. In addition, in some census divisions, the number of observations for some fuel types was very limited, thereby reducing the statistical accuracy of some conclusions. Recognizing that there are some statistical problems it is, nevertheless, possible to make a number of concluding observations on the 1977 data base.

- In terms of square feet of space in the 1977 data base for commercial. and government office buildings, government buildings had relatively less electrically heated space than did the commercial of fice buildings and had relatively more steam heated space.

- Government buildings tended to cluster in age between 10 and 49 years, - with relatively less new or very old space than the commercial sector.

- In general, government buildings use less energy than do commercial buildings, with the exception of steam heated buildings. As was the case for commercial of fice buildings, energy use by government office buildings tended to vary by region of the country and by fuel type.

- Most government buildings were located in downtown areas; government buildings located in suburban areas tended to use more energy than did downtown buildings.

- $79 \%$ of the government buildings were under 10 stories. There does not appear to be any significant trend in energy use as related to height. In comparison, electric use in commercial office buildings did vary by height.

- As was the case with the commercial sector energy use, energy use in government buildings was found to vary as a function of building age.

- Energy consumption also varied with the presence or absence of computer and data processing facilities.

- Data on air conditioning equipment other than electric air conditioning were very limited; however, it was found that the type of air conditioning has had an effect on energy use. 


\section{CONCLUDING COMMENTS}

This report has presented data on office building energy use in 1977 for a sample of commercial and government office buildings based on data collected by the Building Owners and Managers Association for its Experience Exchange Report. The data were subjected to as intensive a review and correction procedure as time and resources would permit, and an effort was made to eliminate buildings with questionable data from the data base. Energy consumption data as measured by Btu/Sq.: Ft. for electric and fuel use were developed in terms of such building characteristics as fuel type, height, age, equipment, and location.

The commercial office buildings in the 1977 data base are representative to a substantial degree of the overall population of buildings in terms of location and age. In terms of quality of space, it is probable that significant amounts of nonprime space are not: included in the data:base. : It is unknown, however, how much non-prime space exists in . the commercial office building sector, and it is, therefore, impossible to evaluate to what degree the buildings are unrepresentative of commercial of fice buildings in general.

A forthcoming report of the Energy Use in Office Buildings project will present an analysis of a data base which BOMA has gathered for a sample of buildings in 20 cities. In that sample, BOMA attempted to gather data for a random, more statistically representative sample of office buildings; it may, therefore, be possible to characterize the building population in 20 cities between prime and non-prime space and to obtain estimates of energy use in the 2 types of space. This information would then permitran evaluation of the data available from the Experience Exchange Report.

The data presented in this report should be a useful input to models for the forecasting of energy consumption. However, 'it should be recognized that the data do not provide a cause and effect explanation of what factors drive energy consumption, nor do the data present energy consumption by end use. The 20 city data base will be subjected to a regression analysis under a subsequent task of this project in an effort to obtain insight on what factors cause energy use to vary. No data are gathered for energy use:by end use, and it will not be possible, therefore, to develop information on energy use by end use in the regression analysis. 
Building energy use simulation programs -- such as the DOE-II, AXCESS, TRACE, Ross Meriwether, etc. -- are typically used to simulate building energy use by end use - heating, cooling, lighting, hot water, fans, pumps, etc. The programs model energy use as a function of the building shell, the building equipment, and building age. It is currently necessary to rely on these types of programs for end use data, for submetering by function is still not practiced to any significant degree in the commercial office building sector.

The data for government office buildings were of lower quality than those for commercial office buildings; 1977 was the first year in which the government buildings participated in the reporting process. Fewer government building observations were available, and the buildings were not representative of the stock of government buildings in terms of age and location. Therefore, the conclusions regarding the government buildings must be viewed as speculative at this time.

The major objective of the Energy Use in Office Buildings subtask presented in this report was to develop the tabular data. As the data were developed, two additional interesting conclusions emerged: there was a decline in office building energy use between 1975 and 1977 , and there is a difference in energy use patterns between commercial and government office buildings. 


\section{APPENDIX I}

QUESTIONNAIRE USED IN COLLECTING

1977 DATA FOR 1978 EXPERIENCE EXCHANGE REPORT 
$\mathbf{r}$

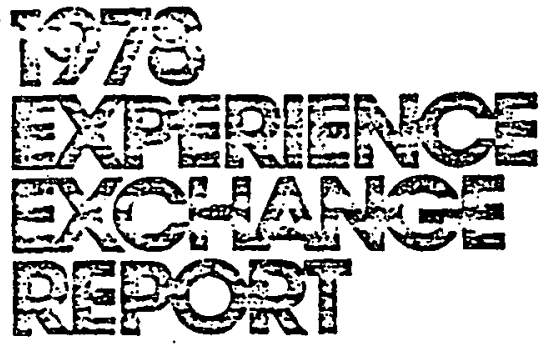

BUILDING

OWNERS

AND

MANAGERS

ASSOCIATION

INTERNATIONAL
1221

Massachusetts Ave. NW

Washington, D.C. 20005

There's a new look to the Experience Exchange Reporr (EER) form this year. But those who have partici. pated in recent years will quickly see that the information requested hasn't changed, except in a few minor instances. The change is in the explanations, given side-by-side with each question for the first time.

Why the big change? Two very important reasons.

First - to make it easier for first-time participants to fill out. Better explanations make it much easier to fumish the requested information. This means it will also be easier and faster for the previous participant to fill out.

Second - to avoid the common misinterpretation of certain questions made in previous years. In processing last year's forms, it was seen that some questions consistently were either left blank or filled in with the wrong type of information. These questions have been rephrased and carefully explained, so it is very important for every previous participant to read the new explanations carefully.

You 11 receive a special computer report worth $\$ 125$ to BOMA members and S225 to non-members for your participation. This report analyzes your building's operations in the same way as the Experience Exchange Report itself. It breaks your data into cents-per-square-foot figures and provides the same useful statistics such as Management Ratio, Operating Ratio and square feet per person shown in the EER tables. It's an extremely valuable document for relating your building to the Experience Exchange Report data. Such a repor requested through the new Operating Performance Comparisons service would cost from $\$ 125$ to $\$ 225$.

A free copy of the BOMA Standard Method of Accounting or the BOMA Standard Method of Floor Measurement is available if you need it to help fll out your report. To obtain either, just write to BOMA International Information Central, 1221 Massachusetts Avenue NW, Washington, DC 20005, mentioning this report form. Because of the cost of printing, handling and mailing, we would ask that you request copies only if you have specific need for it. For members and purchasers of the Experience Exchange Report, both of . these can be found in its back section.

Individual assistance in preparing your report is available from several sources. The executive of your local BOMA Association can put you in contact with members familiar with the report, or if you are in a city where there is no local association, write to Dustin Cole, Communications Manager, at the above address, or call 202.638-2929.

Your local BOMA Association will be kept informed of which buildings in your city have filed reports, so that they can encourage greater participation for your city. This is the only information which will be re!essed; all specific data you furnish remains completely confidential, and is permanently safeguarded.

FIVAL DEADLLNE IS APRIL 1 for completed forms, because of the considerable time necessary for processing, checking and analysis. Help us get this, the most important research project conducted by BOMA International, into the hands of the industry as quickly as possible. Persons submitting the first 100 reports will be named in a special "Honor Roll" in the monthly newsletter; Building Owner and Manager.

Our sincerest thanks in advance for your participation and support.

\section{Hyron Sivarrz}

Chairman

Accounting Commitree

\section{.Jack L. Dierdorff :}

President

BOMA International 
APHENUIX 1

$197 S$ EXPERIENCE EXCHANGE REPORT FOR DOWNTOWN AND SUBURBAN OFFICE BIILDINGS

Report tor (circle one): Calendar year 1977 Fiscal year from to

Building Name

Building Address

City State

Zip

oi Please send Individual Building Analysis to:

Address

City State Zip

Report prepared by

Phone
Date

\section{SCHEDULE I - BUILDING AND MANAGEMENT DATA}

1053 1. STATISTICAL AREAS † Office Area Meosurement method used: BOMA NY NY GSA Other: Retail Area Storage Area - Special Area* - Total Rentable

2. Average 1977 Occupancy: Retail

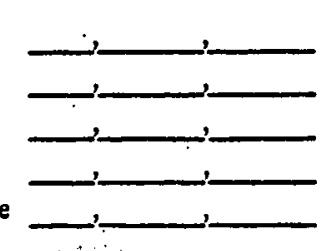
15. Parking: No. Spaces 16-23. Indoor Outdoor 24-31: Contractor-operated _ Run by building \$2:39? Deseribe: $\%$ Office

3. Building Cleaning: done by (You may check one, more than one, or give percentages) Building staff (4) Outside contractor 45. Tenant 46:

4. AREA SERYICED/ MAINTAINED AT EXPENSE OF BUILDINGwhether done by building staff or outside contractor

\begin{tabular}{l} 
Service \\
\hline Cleaning 476 \\
Electrical \\
Heating \\
Air Cond. \\
Plumbing \\
Alterations \\
Repairs \\
Decorating
\end{tabular}

6. Age in years $7+73$
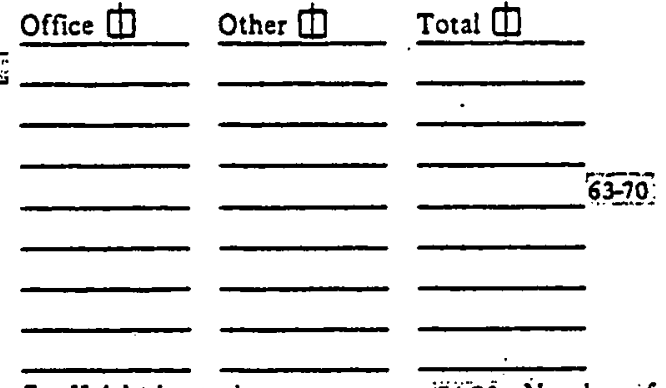

5. Assessed Value for Tax purposes Land $S$ Bldg.5 Total 5 7476 Number of stories below grade

8. Is Building Occupied by owner or lessee of entire building? NO YES./give percent;

9. Office Tenancy: Medical \% Utility \% Bank or S/L $\%$ Government $\%$

10. Is Building Agency Operated? Yes 17 No 11. No. of buildings included in this report?

04 12. Building Population (No. of tenants' employees): Office Area $8-13 \quad$ All other areas 20-23. All other areas $\overrightarrow{2+27}$

13. Number of Tenants: Office Area

$$
\text { : }
$$

15. Annual Payroll for all non-management personnel: $\$$
16. Building-iwned Air Conditioning

\begin{tabular}{ll} 
Type & Tons \\
\hline Central $40-45$ & \\
Package & \\
Other & \\
Annual Depreciation $S$
\end{tabular} Type Energy Used $32-39$ $28-31$ 


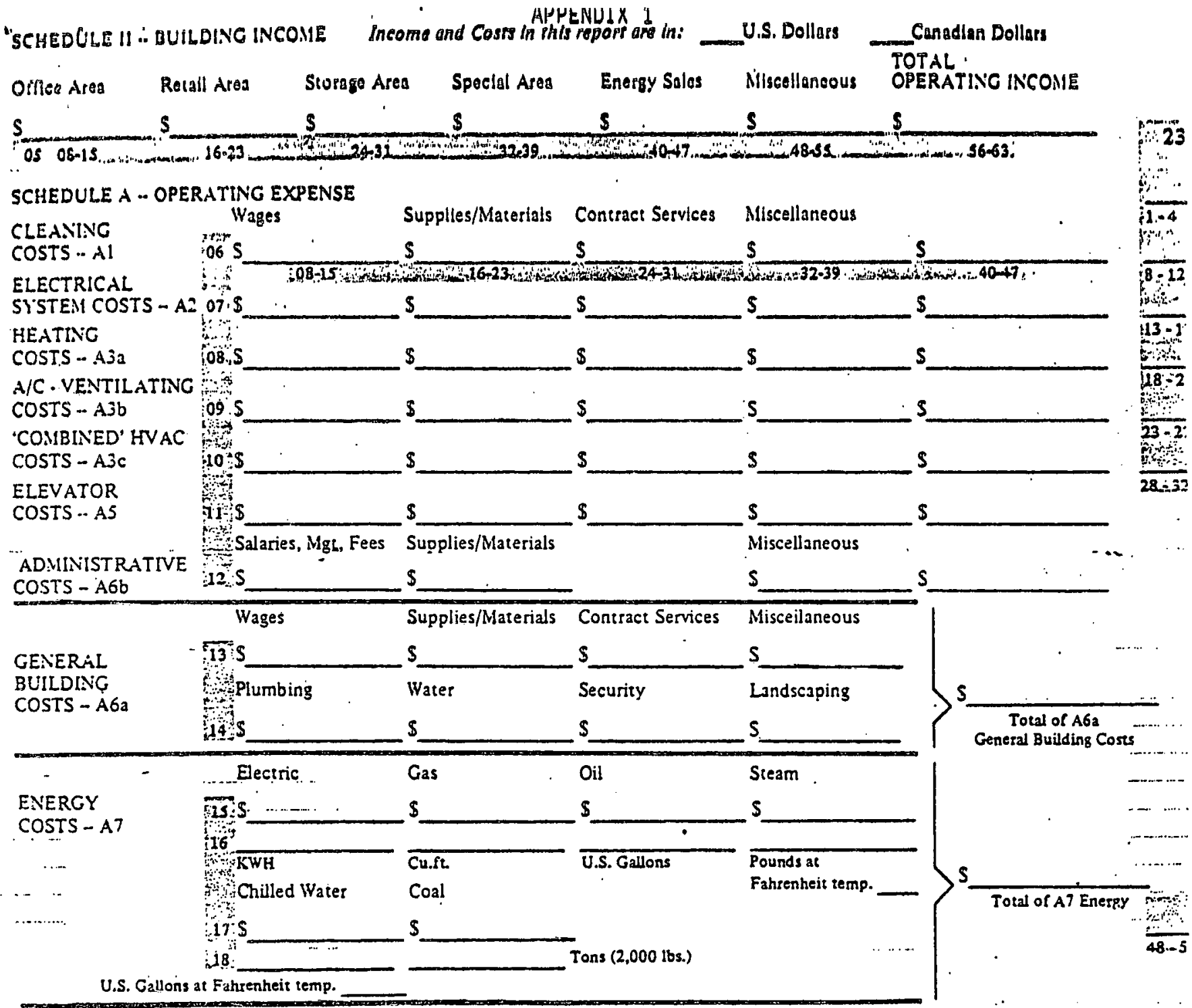

SCHEDULE B - CONSTRUCTION EXPENSE - Expenses for service provided io Tenani Area only.

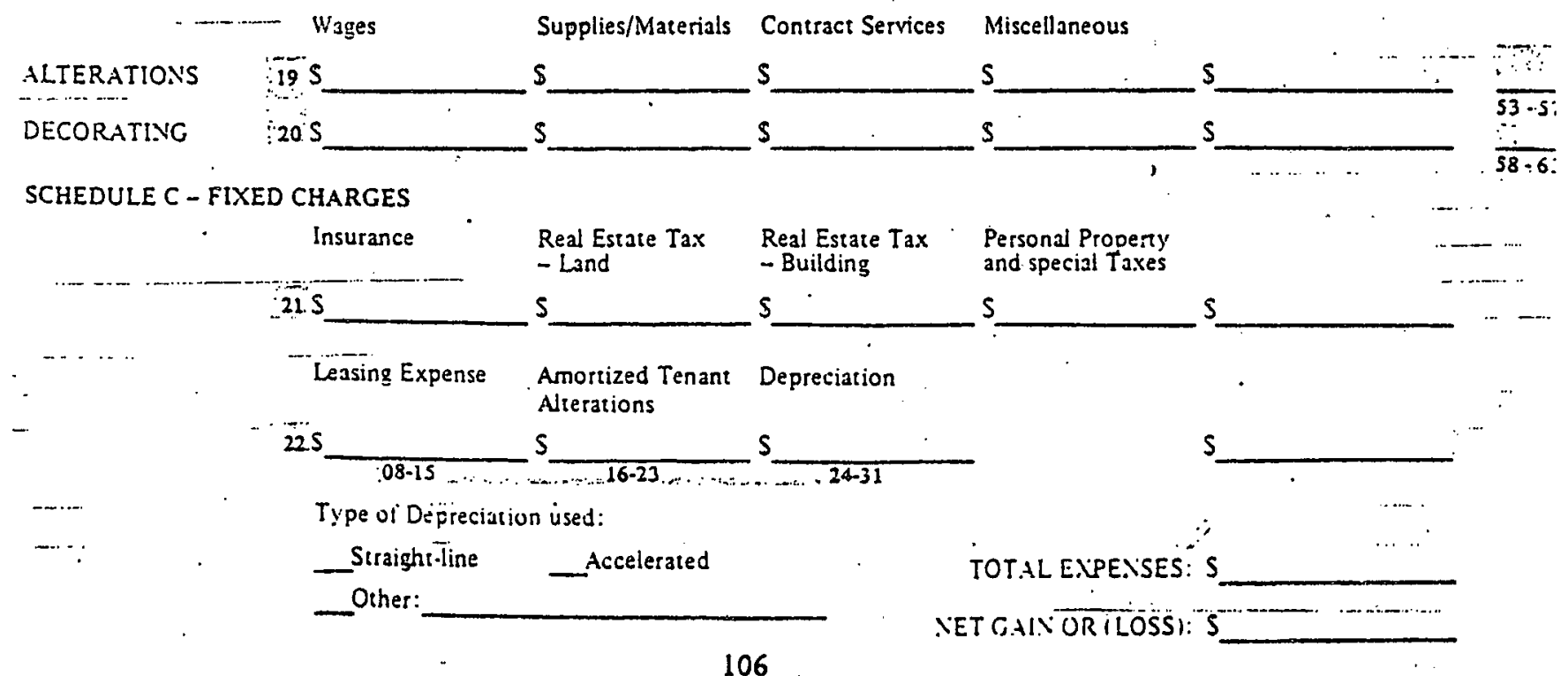




\section{INSTRUCTIONS' FOR' SECTION TIVO}

\section{SCHEDULE A - OPERATING EXPENSE}

Lines Aa through As:

These lines do hop comroin any eneren' costs; th/s is costs for maintenance of the named aystems only. All alterg." cosis ans to be shuwn in line $A 7$.

Woges and Salaries:

For accurate cost accounting it is essential to divide the salarles of persons whose duties encompass more than one area between the appropriate categortes according to percentage of time spent at each. If a specific breakdown is not available, a careful estimase is far more accurate than placing all of these wages into a single category.

Be cerroin to include social security taxes, compensation in. surance, and all fringe benefits in Wages and Salaries.

Al - Cleaning Costs - Report wages and fringes of employees, including supervisory, who clean offices, lobbies, elevator cabs, corridors, toilets, windows, etc.; report cost of all cleaning supplies, contracts for window and other cleaning, upkeep and ordinary replacement of cleaning equipment.

A2 - Electrical Costs - Cost of maintaining building electrical system only. Includes repairs and ordinary replacements, cost of lamps, fuses and other supplies, wages of electricians and other employees who operate and maintain the electrical system.

A3a - Heating Costs - If building has a combined heating/ air conditioning system for which separate costs are not normally obtained, do nor report on this line. Go to $A 3 c$, Combined HVAC. If building has a separate heating system, repor cost of maintaining entire system from coal hole or steam meter to office radiators, including labor costs, sup. plies and parts. Include portion of wages paid engineer, assistant engineer, fireman and any others necessary to operate system.

A3b - Air Conditioning - Ventilating Costs - If building has a combined system which does not facilitate the breakout of heating and $A / C$-ventilating costs, do not use this line. Go to $\mathrm{A} 3 \mathrm{c}$, Combined HVAC. Otherwise, report here all appropriate costs to maintain and operate this system. Remember to include all apportioned wages, materials, etc. on a similar basis to that outlined in $\mathrm{AB}_{\mathrm{a}}$, Heating Costs.

A3c. - Combined . HY AC...Costs - For reporing all costs related to maintenance, operation and supply of an integratedheating/air conditioning/ventilation system.

A5 - Elevator Costs - Repcr wages paid elevator starters and operators, cost of labor for inspecting and servicing elevator system and making ordinary repairs; also all other maintenance costs of elevator equipment, signal system, etc., including supplies, materials and ordinary replacements. A'so expense for uniforms (cost and upkeep). Maintenance contract should be included after making proper proration for extraordinary repairs.

A6b - Adminstrative Costs - Repor all expenses of office building management and clerical salaries, management fees, fees for legal, auditing, appraisal and engineering services, office supplies and equipment, telephone, expense, dues and subscriptions. Include any advertising costs not. relating to space lassing; cláshthá dơvartising for employees, solletication for maintenance, supply contracts, etc. Do not include: ad. vertising for lessing purposes, leasing commissions or dis. counts, or any spacs marketing expense; financlal charges such as corporate saxes, or organization expense, execu. tive/corporate salaries and expenses.

A6a - General Building Costs - Please note carefuily that there are two lines for data; second line requests specific dara for subcategories of Plumbing, Water, Security, and Land. scaping; here, add all components of each mwages, supplies/ materials, contract services and miscellaneous - into a total. and enter appropriately. First line requests the normal breakout of those components for all other General Buiding Costs not charged to previous sub-categories. Include: keys, lettering on doors, outdoor or public area cleaning, general repairs, directory service, civilian defense preparation costs, etc.

A7 - Energy Costs - Cost and quantity of each type of energy used for building operations and provided or sold to tenants. F temperature on both Steam and Chilled Water must be furnished in order to make any calculation of $\mathrm{BTU} / \mathrm{sq}$. ft. possible. Important: If any portion of your space buys direct from utility, costs and quantities must be obtained and added to totals to produce meaningful energy use figures for your building.

\section{SCHEDULE B - CONSTRUCTION EXPENSE}

Alterations, Tenant Area - Cost of minor alterations made in tenant areas, which are assumed by building.

Decorating, Tenant Area - Costs of the normal periodic major cleaning and decorating of offices, refinishing of floors, etc. when paid for by building. Costs of decorating necessitated by alterations should be reported under Alterations, Tenant Area if paid for by building.

\section{SCHEDULE C - FIXED CHARGES}

Insurance - Cost of all types of 'building' insurance such as fire, extended coverage, liability and water damage.

Personal Property and Special Taxes - Costs only for personal property taxes and special licenses.

Leasing Expense - Report cost of broker commissions, consultant fees, advertising done for space rental, etc., which relate to leasing of space in building.

Amortized Tenant Alterations - Report annual charge (pro. ration) of major tenant alteration costs, including decora. tions, paid for by building and amorized over the terms of the leases.

Depreciation - Report here charges for depreciation on the building proper, as well as on all machinery, mechanical equipment, furniture and fixtures. Aso, please indicate type of depreciation used.

BOMA is working to forestall the possible require. ment for office buildings to report energy-use directly to the federal government. For this, better comparison information for the base year 1972 has been requested. Plcase lielp by providing ynur infor. mation in the space on the back of the EER form. 
Before heginning to enter dara. please read through the entire form and the explanations which apply to each question. Remember that correlation of data between schedules is essential if accurate relationships are to be shown on both your Individual Building Analysis and the Experience Exchange Reporr tables.

\section{GENERAL INSTRUCTIONS}

This report form has been color-keyed to assist you in its preparation. Blue indicates account and sub-account titles and black shows general questions and bianks for dollar entries.

Please use dollar amounts only. Do not enter cents on the form. If there is no expense under a heading, please indicate with -0 - rather than leaving it blank.

All shaded areas are for computer codings only, and require no entries by you.

To receive your Individual Building Analysis, please make sure to fill in that section. It is also very important that the person who actually fills out the report give his or her nane and telephone number on the appropriate line, so that he may be reached if there is a question concerning an entry.

\section{SCHEDULE I - BUILDLNG AND MANAGEMENT DATA}

1. Stutistical areas: To calculate all other figure: correctly, a permanent statistical area must be calculated for each of the rentable building areas. This is done on a "Single Tenancy Floor' basis. This means that regardless of how a floor is subdivided for multiple tenants, calculations are always based on the 'potentially rentable' area. These statistical areas will not change for the life of the building unless actual structural (not remodeling) changes are made in the building. Be sure to indicate the method of floor measurement used to calculate areas.

Statistical Office Area - Report the total rentable office area in the buiding, calculated on a Single Tenancy Floor basis.

Statistical Retail Area - Report the total area leased or available for lease as Retail Area. This is nomally the ground floot area but may be other locations as well, such as underground, or at upper-level breezeways.

Statistical Special Area - Area leased, used or available for leasing for special purposes such as corporate cafe. terias, private clubs, restaurants, etc., which are not on the ground floor of the building.

2. Average 1977 Occupancy - Please average your monthly. occupancy rates. This is a very imporant figure used to factor your costs and income.

4. Area Serviced at Expense of Building - This is extremely imporant to all allocations of expense between building rentable and office rentable areas. This indicates the allo. cation of 'Maintenance' costs and not Energy. Please indicate the square footage of the actual statistical area served for each service listed.

6. Age in years: From date building was first available for occupancy.
9. Office Tenancy: Do not include any ground noor 'retail' area doctor's offices or bank.S/L branches.

11. Number of Buildings: If your accounting system is for several buildings on a consolidated basis and you are unable to break them out separately, indicate the actual number of buildings being reported on. Similarly, all statistical areas should show consolidated figures. If more than one building is in report, show averaged age and height in questions 6 and 7.

12. Building Population: Workers in building, not including building employees. If owner-occupied, exclude all personnel equivalent to "building operating staff.

14. Building Employees: Wage-rate personnel. Do not include any salaried unless they are actual operations (as opposed to management) personnel.

15. Annual Payroll, non-management: Payroll for the personnel shown on question 14.

16. Building-owned air conditioning: Do not include any units owned and maintained by tenants. Type Energy Used: electric, gas, steam, etc. Package: Under 'Tons' please show total tonnage of all, rather than per unit.

17. Building location: Definition of Central Business District may be obtained from your local BOMA Executive or your Chamber of Commerce.

19. Building Electrical Policy: Please give percentages based on square feet of each in relation to total rentable.

\section{SECTION TWO - INCOME AND EXPENSE DATA}

\section{SCHEDULE II - BUILDING INCOME}

Office, Retail, Storage, and Special Areas

For general definitions of these areas, see the explanatory notes for Schedule I, question 1, Statistical dreas.

Office Area - Imporrant: space occupied by owner/lessee of building must be included. If space is not charged at fair market value, use cents/sq. ft. averages of rents, as escalated, and charges paìd during the year by building tenants.

Energy Sales - Report the gross income from sales to tenants or any other purchaser of electricity or any form of energy (such as steam, chilled water) reported under the 'Energy Costs A7 line below.

Miscellaneous - Report interest earned, cash discounts. recovery of bad debts, sale of waste paper and all other income not reported elsewhere. Include here the gross of all income for all services paid for by tenants which is not included in rent, if these were performed by regular or contract personnel and if the costs for these are included in some part of Schedule A - Operating Expense. If the costs are not included in any Operating Expense categon;, report only the net income. Such 'extra' services to tenants include above normal "building standard" cleaning, waxing of foors, special security services, special electrical or plumbing tacilities, repair of tenants' furnishings, etc.

Income from Escalator or Rent Adjustment Clauses:

Report such income in individual rental income categories 10 which it applies. such as Office Area. Retail Area. etc.

COITISLED IISIDE. 


\section{YOUR 1972 ENERGY INFORMATION IS URGENTLY REQUESTED}

If your trade association can provide energy information in sufficient detail to answer the requests of the federal government, we may be able to forestall any requirement for individual buildings to report directly to the government. Your information for the 'base', or comparison, year of 1972 can be of major help in providing the requested information on an aggregate basis, protecting the confidentiality of all reports. Help your industry and yourself; please take the time to obtain your 1972 data and provide it here.

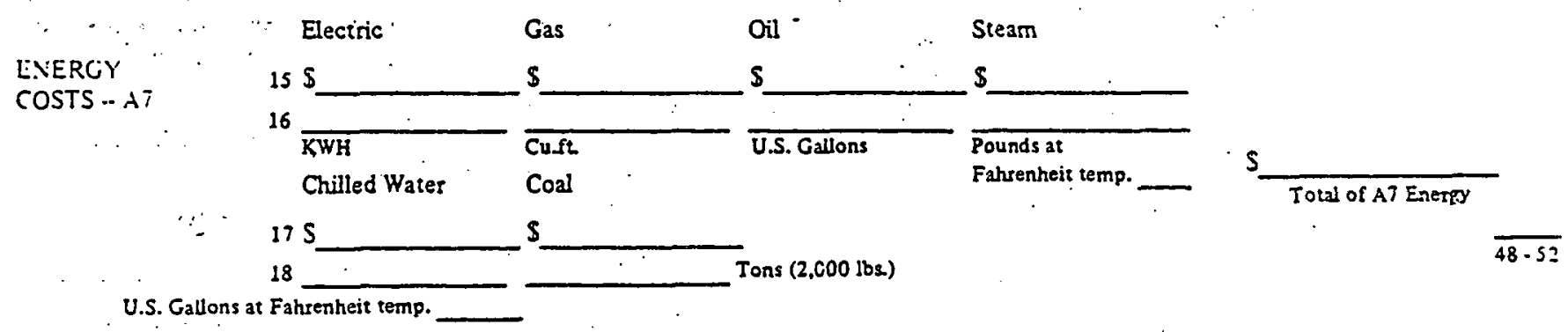

TO SAVE YOU TLME, this form is pre-addressed and designed for retum in 3 window envelope.

DETACH INSTRUCTION SHEET and fold as shown to mail.

AGAIN. OUR SINCERE THANKS for your participation.

1978 Experience Exchange Report BOMA International

1221 Massachusetts Avenue, NW

Washington, DC $20005^{\circ}$ 
THIS PAGE

\section{WAS INTENTIONALLY LEFT BLANK}




\section{CHARACTERISTICS OF THE DATA BASE}

1. There are 36 cards per building.

2. Each card has a building number, cols 1-4, and a card number cols 5 and 6 .

3. The next 19 colums, cols $7-25$, contain a vector called NEWREC. NEWREC contains the following variables:

a. U.S. census division number, col 7. For a listing of the numbering system for the census divisions, see Attachment 1.

b. BOMA region, Col 8, and BOMA city, Cols 9 and 10. For a listing of BOMA regions and cities, see Attachment 2. 
c. Population code of each city, col 11. The codes are as follows:

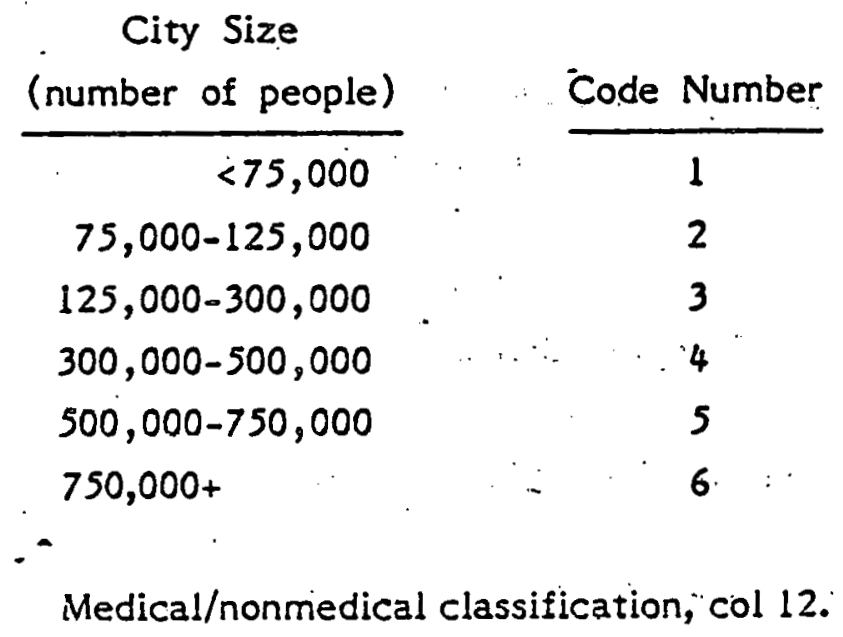

The entry "O" denotes a nonmedical building. Any other entry denotes a medical building.

e. Building age, cols 13-15.

f. Building height in stories, cols 16-18.

g. Building size code, col 19.

\begin{tabular}{lc}
$\begin{array}{l}\text { Building Size } \\
\text { (square feet) }\end{array}$ & Size Code \\
\hline 50,000 & 1 \\
$50,000-100,000$ & 2 \\
$100,000-300,000$ & 3 \\
$300,000-600,000$ & 4 \\
$600,000+$ & 5
\end{tabular}

h. Building downtown/suburban location, col 20.

i. Building air conditioning, col 21 . "O" denotes that the building is air conditioned. 
j. Government/Private Ownership, col 22.

"O" denotes that the building is privately owned; "G" denotes government ownership.

k. Single Purpose Building Code, Col 23.

"O" denotes that the building is not single purpose.

1. Agency Management Code, Col 24.

"O" denotes that the building is not managed by an agency.

m. Number of buildings in report, $\operatorname{col} 25$.

4. The remainder of Card 1 contains the first 3 numbers of a $1 \times 213$ vector, REC(I). The 3 numbers are found in cols 26-35, 36-45, 46-55. All elements of the vector REC(I) are defined in Attachment 3.

5. Cards 2 through 31 contain the remainder of REC(I). Each of the cards 2 through 31 has the following format:

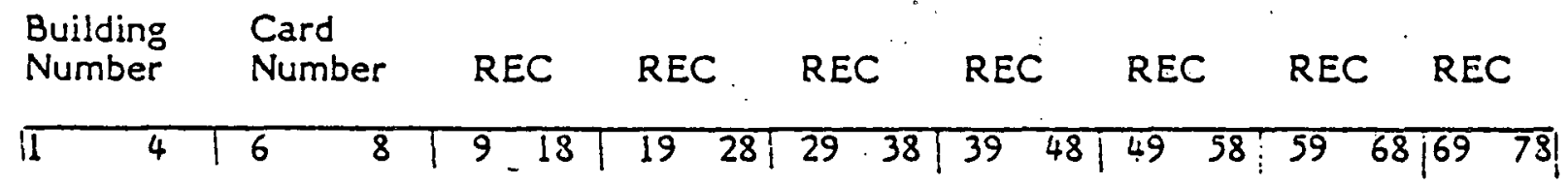

6. Card 32 contains the following data:

a. Building number, Cols $1-4$

b. Card number, Cols 5-6

c. Predominant energy purchased to heat the building: $\mathrm{Col} 7$. Col 7 is coded as follows:

$\begin{array}{ll}\text { Electricity } & =1 \\ \text { Gas } & =2 \\ \text { Oil } & =3 \\ \text { Steam } & =4 .\end{array}$


d. Alternative Fuel Capabilities (if any), col. 8.

$\begin{array}{ll}\text { None } & =0 \\ \text { Electricity } & =1 \\ \text { Gas } & =2 \\ \text { Oil } & =3 \\ \text { Steam } & =4 .\end{array}$

e. Grade of oil used, Col 9 .

\# 2 distillate $=1$.

\#6 residual $=2$ 。

f. Square feet of computer space in the building, Cols 10-15. Such space is counted only where special alterations, such as a raised flooring or additional heating, ventilating, and air conditioning; is required.

g. Tons of electric air conditioning in the building, Cols 16-20. If window units or a window and central combination are used, the total combined tonnage of all electrical air conditioning equipment is presented.

h. Tons of steam turbine air conditioning in the building, Cols 21-25.

i. Tons of steam absorption air conditioning, Cols 26-30.

j. Whether data are estimated or actual for fuel quantities, cols 31 36. " 1 " denotes estimated, and " 2 " denotes actual.

$\begin{array}{ll}\text { Electricity } & \operatorname{col} 31 \\ \text { Gas } & \operatorname{col} 32 \\ \text { Oil } & \operatorname{col} 33 \\ \text { Steam } & \operatorname{col} 34 \\ \text { Chilled Water } & \operatorname{col} 35 \\ \text { Coal } & \operatorname{col} 36\end{array}$

k. Whether building is all electric; "l" denotes "maybe" (BOMA uncertain); "2" denotes "yes". Any other entry denotes "no". 
7. Card 33 contains real quantity energy data in the following format:

\begin{tabular}{|c|c|c|c|c|c|}
\hline $\begin{array}{l}\text { Building } \\
\text { Number }\end{array}$ & $\begin{array}{c}\text { Card } \\
\text { Number }\end{array}$ & $\begin{array}{c}\text { Electricity } \\
\text { kwh } \\
(x 1000)\end{array}$ & $\begin{array}{c}\text { Gas } \\
\text { cu ft } \\
(\times 1000)\end{array}$ & $\begin{array}{c}\text { Oil } \\
\text { gal } \\
(\times 1000)\end{array}$ & $\begin{array}{l}\text { Steam } \\
\text { Pounds } \\
(x 1000)\end{array}$ \\
\hline . & 6 & 14 & 1522 & $23 \quad 30$ & $31 \quad 38$ \\
\hline
\end{tabular}

8. Card 34 contains more real quantity data, in the following format:

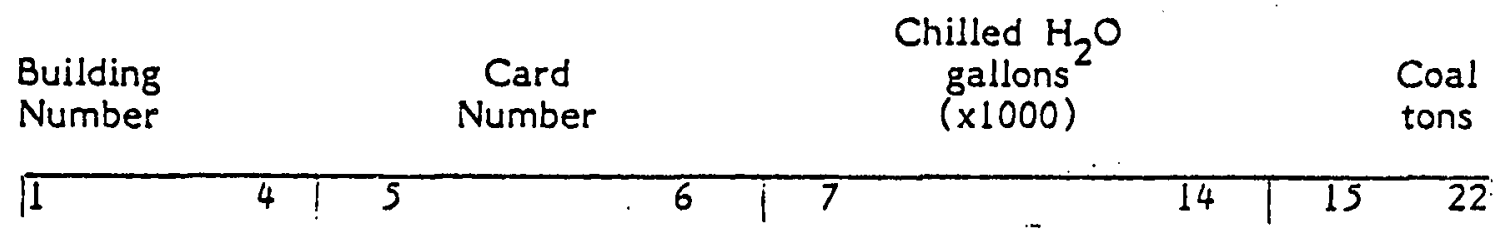

9. Card 35 contains BTUs (Total) for electricity, gas, oil, steam, coal, all non-electricity, total energy use. The format is as follows:

Building Card
Number Number Electricity Gas Oil Steam Coal Electrical Total

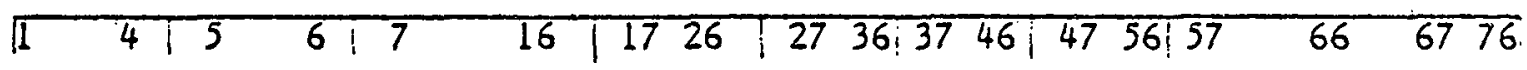

The following conversion factors were used to convert physical quantities into BTU's:

$\begin{array}{lll}\text { Electricity } & : & 3412 \mathrm{BTU} / \mathrm{kwh} \\ \text { Gas } & : & 1020 \mathrm{BTU} / \mathrm{Cu} \text { ft. } \\ \text { Oil, 非 } & : & 138,690 \mathrm{BTU} / \mathrm{gallon} \\ \text { Oil, 非 } & : & 149,690 \mathrm{BTU} / \mathrm{gallon}\end{array}$

In cases in which oil type was not specified, it was assumed that $\$ 2$ oil was used by the building.

Steam : $1000 \mathrm{BTU} / \mathrm{cu}$. ft.

Coal : No building uses coal

10. Card 36, in the same format as card 35, presents BTU's per square foot of total building area (in square feet) for corresponding quantities. 
11. There are no census numbers associated with government buildings, since government buildings are coced by BOMA region and not by city. In the data base furnished by BOMA, all builüings were numbered sequentially. Attachment $4 \mathrm{a}$ cross references the sequential numbering system with BOMA's internal numbering system. Attachment $4 b$ lists the city location for each government building. 
Census Division

New England Division

Mąine

New Hempshire

Vermont

Messachusetts

Rhode Island

Connecticut

Middle Átlantic Division:

New York

New Jersey

Pennsylvania

South Ailantic Division.

Delaware

Maryland

Disirict of Columbia

Virginia

West Virginia

North Carolina

South Carolina

Georgia

Florida

East North Central Division:

Ohio

Indiana

Ulinois

Michigan

Wisconsin

East South Central Division:

Keniucky

Tennessee

Alabama

Mississippi

$\frac{\text { Number }}{1}$

2

Census Division

Number

West North Ceniral Division

6

Minnesota

lowa

Missouri

North Dakota

South Dakota

Nebraska

Kansas

West South Ceniral Division: 7

Arkanses

Louisiana

3

Oklahoma

Texas

Mountain Division: $\quad 8$

Montana

Idaho

Wyoming

Colorado

New Mexico

Arizona

Utah

Nevada

4

Pacifie Division: 9

Washington

Oregon

California

Aleska

Hawaii 